\title{
Cell adhesion by integrins
}

${ }^{1}$ Michael Bachmann,$+{ }^{2}$ Sampo Kukkurainen,$+{ }^{2}$ Vesa P. Hytönen, ${ }^{1}$ Bernhard Wehrle-Haller*

${ }^{1}$ Department of Cell Physiology and Metabolism, University of Geneva, Centre

Médical Universitaire, Rue Michel-Servet 1, 1211 Geneva 4, Switzerland

${ }^{2}$ Faculty of Medicine and Health Technology, Tampere University, Tampere, Finland, and Fimlab Laboratories, Tampere, Finland

* Corresponding author: bernhard.wehrle-haller@unige.ch

+ Shared first authors

\section{Abstract}

Integrins are heterodimeric cell surface receptors ensuring the mechanical connection between cells and the extracellular matrix. In addition to the anchorage of cells to the extracellular matrix, these receptors have critical functions in intracellular signaling, but are also taking center stage in many physiological and pathological conditions. In this review we provide some historical, structural and physiological notes, so that the diverse functions of these receptors can be appreciated and put into the context of the emerging field of mechanobiology. We propose that the exciting journey of the exploration of these receptors will continue for at least another new generation of researchers. 


\section{Chapter 1: Introduction and some historical notes}

It is always difficult to trace back the origin of an idea, a particular historic event or the role of its founders, that initiated a new way of thinking in a particular field of science. In the case of the cell-matrix adhesion receptors of the integrin family, we could highlight the work of Abercrombie and co-workers as well as Curtis, who explored the mechanisms allowing cells to adhere to and crawl on petri dishes, recognizing the cytoskeleton and substrate anchoring adhesion sites visible in the electron microscope or by interference reflection contrast $(1,2,96)$. Cell adhesion was also a subject interesting researchers in the field of tumor biology, as a central feature of cancer cells is their ability to grow on soft agar, indicating that these cells no longer require adhesion to their tissue environment and have lost the regulatory influence of the healthy microenvironment of the tissue (273). At about that time, Richard Hynes incubated normal adhering hamster fibroblasts or their hamster sarcoma virus-transformed derivatives with an extracellular iodination solution. When analyzing the iodinated proteins by SDSPAGE, he identified an abundant $250 \mathrm{kDa}$ protein present on normal, but not on transformed cells (202). This large, external, and transformation-sensitive (LETS) glycoprotein was simultaneously found and characterized in many different laboratories and given names such as cold-insoluble globulin, cell surface protein, fibroblast surface antigen and eventually named fibronectin $(296,375,474)$. Since fibronectin showed an intriguing overlap with intracellular stress fibers (204), the existence of a transmembrane link was postulated. Only a few years later it became clear that fibronectin was a major extracellular binding partner for fibroblasts and that the critical binding element in fibronectin was a short peptide Arg-Gly-Asp (RGD) (7, 334). The respective surface receptors recognizing this motif in fibronectin as well as in vitronectin were identified by Pytela and Ruoslahti $(349,350)$. In an alternative approach, the same fibronectin-binding surface receptors were also identified based on monoclonal antibodies that prevented cell binding to fibronectin, such as JG22, CSAT and GP135 (8, 97, 158). Shortly afterwards the integrin field enjoyed its first expansion phase, where all the different integrin receptors and the majority of their ligands were described and named, either according to biochemical or ligand-affinity data as in the case of fibronectin ( $\alpha 5 \beta 1)$ (349) and vitronectin ( $\alpha v \beta 3$ ) receptors (350), or by researchers working in the field of immunology according to antibody reactivity as for VLA1 to 6. Especially the latter field helped to develop the concepts of integrin-dependent adhesion during platelet activation or cytokine-mediated adhesion of leukocytes to the endothelium via ( $\alpha 4 \beta 1 /$ VCAM-1) $(110,426)$ and LFA1 ( $\alpha$ L $\beta 2) /$ ICAM-1 binding $(117,383,406)$. Importantly, these integrin-dependent adhesion processes were not constitutive, but could be triggered by cytokine stimulation and even $\beta 1$-integrin-directed 
adhesion-stimulating antibodies, proposing that the affinity of these cell surface receptors was specifically regulated (22). The analysis of integrin receptors and their ligand specificity on the vascular endothelium (85) eventually led to the idea, that the inhibition of integrin-dependent adhesions in sprouting endothelial cells could inhibit the angiogenic switch and prevent tumors from growing in the tissue $(59,138,209)$, taking the research on integrin receptors to almost all domains of biomedical research.

One of us was actually in the lucky position to assist this process, as his colleagues were actively identifying, purifying and characterizing different members of the integrin family in the labs of Jürgen Engel, Mats Paulsson and Ruth and Matthias Chiquet (193, 298, 423). It was clearly the golden age, or alternative the "Sturm und Drang" period, of the integrin and extracellular matrix research, in which most of the integrin-receptor concepts were created. In this phase also the majority of the integrin knockout models were established in the labs of Richard Hynes, Reinhard Fässler, Dean Sheppard and many others (111, 132, 198, 396), leading to the quintessential integrin review published in 2002 by Richard Hynes (203).

About this time, first attempts were made to understand the structure-function relationship of integrin receptors. First, the I-domain insert of the $\alpha$-subdomain of the lymphocyte integrins $(\alpha \mathrm{M})$ was crystallized in two different conformations, providing a strong argument for the association of integrin ligand binding with conformational changes in the receptor (245). While the I-domain of the $\alpha$-subunit exhibited a single metal-ion-dependent ligand binding site, the revelation of the structure of the entire extracellular domain of the $\alpha v \beta 3$ integrin receptor, identifying three differently complexed metal ions coordinating the RGD-peptide to the central $\mathrm{Mg} 2+$ ion, determined a breakthrough in understanding how integrin-ligand-binding was coupled to conformational changes of the integrin receptors $(471,472)$. The structural differences between the headpiece of the lymphocyte integrin $\alpha \mathrm{M}$ and the integrin $\alpha v \beta 3$ expressed in fibroblasts and endothelial cells allowed first considerations about the connection of integrin structure to physiological function (see Chapter 2).

It took a few additional years to understand the flexible elements of the integrins and the allosteric conditions under which the receptor was extending into a conformation that was compatible with ligand binding (470). Importantly the crystallographic studies with the $\alpha$ IIb $\beta 3$ integrin ectodomain were backed up by electron microscopy analysis of individual integrin heterodimers changing their conformation in the presence of $\mathrm{Mn}^{2+}$ ions and RGD peptides, 
confirming the allosteric nature of the integrin receptor (125). With the analysis of other integrin receptors, however, the debate continues about how conformational flexibility of the integrin receptor and allosteric influence of intracellular adapters and extracellular ligands shape the function of the different integrin receptors $(289,484)$ (see Chapter 2).

This second phase of in-depth analysis of the integrin structure/function relation was greatly advanced by the discovery of the green fluorescent protein (GFP). The fusion of GFP to cytoskeleton proteins or integrins allowed to localize these receptors in living cells, to study their dynamic association in the plasma membrane and their cycling through the membrane systems of the cell. In migrating cells, a different behavior of $\beta 3$-GFP-integrin clusters located at the front and at the rear of cells was apparent (35). Furthermore, the differences in the integrin cluster behavior between immobile, but transient clusters in the cell front, and inward sliding integrin clusters at the cell rear correlated with the dynamic exchange measured by fluorescence recovery after photobleaching (FRAP) between these different integrin-dependent adhesions. Interestingly, the dynamics of the integrin exchange depended on the regulation of the actin cytoskeleton, providing at the same time a structural and dynamic vision of the integrin receptors and their association with the actin cytoskeleton and integrin adaptor proteins such as talin and vinculin $(35,90)$.

However, as we are learning more and more about the different integrin receptors, their functions as well as mechanical and signaling capacities, we have entered a third and still ongoing phase of research on the integrin receptor family. This third phase involves attempts to integrate the notion of mechanosignaling with the mechanical aspects of cell linkage to the extracellular matrix. Tensional forces created between the extracellular matrix and the cytoskeleton induce changes in the extracellular visco-elastic scaffold, the integrin receptors as well as their adapter proteins, linking intracellular signaling to conformational changes in multidomain proteins $(205,450)$. In turn, such conformational changes can affect enzymatic reactions and lead to activation of kinases such as focal adhesion kinase (FAK) and src family kinases as well as different types of phosphatases. Thus, the large number of integrin-associated proteins, defined as the adhesome $(63,239,387,492)$, as well as their differential interaction with the plasma membrane is forming a puzzle consisting of 200 to 1000 different pieces, of which we have only limited structural and biochemical information. Under tensional stress many of these adhesome proteins will undergo conformational changes, further increasing the complexity of the adhesion site. It remains a challenging task to identify the molecular 
machinery, that has constantly evolved since the moment cells started to actively explore their environment and to form multicellular organism relying on extracellular scaffolds.

\section{Chapter 2: Structure and allosteric control of the integrin receptor}

\section{Overall integrin structure}

As mentioned above, some integrins like $\alpha \operatorname{IIb} \beta 3, \alpha \mathrm{V} \beta 3$, and the integrins involved in immunological functions containing the $\beta 2$ subunit have been studied in more detail than other members of the family, and many concepts in the field are based on these integrins. We therefore want to give a general overview about the structural organization of integrins before a more detailed discussion about structure and integrin activation based on $\alpha \operatorname{IIb} \beta 3$ and $\alpha \mathrm{V} \beta 3$ integrins. Finally, we extend the discussion to other integrins and the differences in their organization before presenting potential consequences of integrin structure for their physiological function (Chapter 3).

Ultimately, the understanding of the physiological roles of integrins requires to comprehend the link of structural organization to adhesive function. Especially crystallography, electron microscopy (EM), and conformation-specific antibodies have been pivotal to reveal different conformations of integrins and the structural organization of the $\alpha$ - and $\beta$-subunits (Figure 1). Both subunits are tightly bound to each other by interactions between the $\alpha$-propeller and the $\beta$-I-like domain in the extracellular "head" regions of both subunits. This association occurs in the endoplasmic reticulum, and single chain integrins do not reach the cell surface (250). Probably the most drastic structural difference between integrins is the presence or absence of the ligand-binding $\alpha$-I domain, inserted in the top part of the $\alpha$-subunit ( 9 integrins have, 15 do not have an $\alpha$-I domain; see Figure 1). The $\alpha$-I and $\beta$-I-like domains are structurally related to the Von Willebrand factor A-domain, exhibiting both a metal ion-dependent adhesion site (463). Although showing a similar fold, the $\beta$-I-like domain in the $\beta$-subunit of integrins possesses some unique structural characteristics (see below). Integrins with an $\alpha$-I domain belong to the classes of collagen-binding integrins and leukocyte specific integrins (Figure 1) and are found only in vertebrates. Functionally, the most obvious difference between integrins with and without $\alpha-\mathrm{I}$ domains is the mode of ligand binding. Integrins without $\alpha-\mathrm{I}$ domains bind ligands in a binding pocket formed by the $\alpha$-propeller in the $\alpha$-subunit headpiece and the MIDAS ion in the center of the $\beta$-I-like domain of the $\beta$-subunit (Figure 2, 3). In contrast, integrins with an $\alpha-\mathrm{I}$ domain recognize ligands only with their $\alpha$-I domain, which is however 
structurally coupled to a $\beta$-I-like domain-binding "IEGT" peptide motif, serving as an internal integrin ligand (Figure 3D). Further analysis showed that the spatial arrangement of ligandintegrin interactions is diverse even within the respective groups of integrins with or without $\alpha$-I domains (Figure 3).

Given the frequency of the RGD sequence in many extracellular matrix proteins, the group of RGD-binding integrins is considered to recognize many different ligands. Because of the importance of the RGD sequence motif, one might neglect the relevance of the structural organization around the RGD peptide and the respective specificity of the ligand binding event. While present in an exposed loop in fibronectin, the RGD-peptide is flanked by a helical motif in the latent TGF- $\beta$ binding protein, which leads to the specificity in binding to $\alpha v \beta 6$ and $\alpha v \beta 8$ $(113,321,484)$. In addition, the initial characterization of integrin-ligand binding specificity proposed the selective recruitment of the RGD ligands vitronectin and fibronectin to $\alpha \mathrm{v} \beta 3$ and $\alpha 5 \beta 1$ respectively $(349,350)$. More recently, we have revisited ligand specificity by creating binary choice substrates, that allow cells to simultaneously use their different integrin populations on the most relevant ECM ligand (335). In fact, when cells were given the choice between different substrates, the selection of the appropriate ligand was surprisingly specific, suggesting that cells prefer to adhere on the most fitting adhesive surface in respect to ligand density and stiffness. However, cells were also able to adhere to less-preferred ligands, indicating that flexibility in ligand recognition might explain seemingly promiscuous integrinligand binding. New techniques, e.g. single cell force measurements (233) and super-resolution light microscopy (292) can detect differential ligand interaction in living cells (373), and will certainly facilitate the reassessment of integrin-ligand interactions, their dynamic regulation, and their in vivo behavior.

The ligand-recognizing headpieces of both $\alpha$ - and $\beta$-subunits are sitting on top of "leg" domains (Figure 2), followed by transmembrane regions and, with the exception of $\beta 4$ integrin, comparably short cytoplasmic tails. While the extracellular headpiece binds ligands, the cytoplasmic tails interacts with intracellular adapters. Especially the cytoplasmic tails of $\beta$-subunits have been analyzed in detail and are attributed to important functions in regulating integrin activity (see below) and actin linkage (Chapter 4). Functions and binding partners of the cytoplasmic tails of $\alpha$-subunits are less studied and have been associated with integrin inactivation rather than activation and signaling (see Chapter 4 and $(54,344,359)$ ). 


\section{$\alpha I I b \beta 3$ and $\alpha V \beta 3$ integrin activation}

Integrin activation (in terms of gaining the ability to bind ligands) is coupled to extensive structural changes in both subunits. Currently, the prevailing model for $\alpha \operatorname{Ilb} \beta 3$ and $\alpha \mathrm{V} \beta 3$ integrin activation assumes a tight coupling of integrin-ligand binding with a structural change from a bent-closed to an extended-closed integrin conformation ('switchblade model'; similar to the opening of a Swiss army knife), and a further opening of the head piece to an extendedopen conformation (Figure 3E). All three conformations are present in the membrane in a dynamically regulated equilibrium that involves intracellular adapters, as well as extracellular ligands. Ligand binding affinity increases with integrin extension and head-piece opening. However, also the bent conformation is able to bind RGD ligands (472). Nevertheless, the structural rearrangement during integrin extension and subsequent head-piece opening is accompanied by several local changes in the headpiece of the $\beta$-subunit induced by the carboxyl binding of the Asp-side chain of the RGD-motif to the central MIDAS $\mathrm{Mg}^{2+}$ ion (Figure 3A,B): (i) the ADMIDAS site moves towards the Asp-bound $\mathrm{Mg}^{2+}$ ion, (ii) the $\alpha 1$-helix in the $\beta$-I-like domain straightens, (iii) the $\alpha 7$-helix makes a piston-like movement towards the hybrid domain, (iv) which swings out, thereby increasing the angle to the $\beta$-I-like domain and completing the headpiece opening (Figure 3). It seems that these discrete structural events cannot be uncoupled during the process of headpiece opening; straightening the $\alpha 1$-helix by mutations leads to increased overall integrin activation (495), as does constitutive hybrid domain swing-out by introducing a glycosylation site that provokes opening of the angle between the $\beta$-I-like and hybrid domain by steric interference (Figure 3A) (272). The structural integrin activation process starts with a bent state and proceeds to the extended-closed and finally to the extendedopen state $(347,427,500)$. In contrast to such a strict three-step process, Zhu and colleagues showed that headpiece opening of $\alpha \operatorname{Ilb} \beta 3$ integrin is a continuous process, in which they defined eight different steps (501). They also estimated the integrin headpiece affinity for an RGD peptide in the open state to be more than 200-fold higher than in the closed conformation and thus considered the extended-open conformation to be the active, ligand-binding state. Moreover, recent electron microscopy data of different $\beta 1$-integrin containing integrins, proposes that the bent-closed conformation is not typical for these integrins, but regulated essentially at the level of the integrin head-piece opening $(289,417)$. In addition, recent data from our group indicates at least for $\alpha \mathrm{V} \beta 3$ integrin, that the correlation between conformation and ligand binding is more complex: $\alpha \mathrm{V} \beta 3$ integrin locked in the extended-closed state was able to bind vitronectin, but not fibronectin. Only the extended-open state of $\alpha \mathrm{V} \beta 3$ integrin was able to bind fibronectin, a behavior that required tensional forces acting on the integrin receptor 
(31). Thus, structure-function relationships differ among ligands binding the same integrin, suggesting that the extended-closed conformation might be more than just a 'not yet activated' integrin. A similar situation was demonstrated for $\alpha 4 \beta 7$ integrin, where two cytokines (CCL25 and CXCL10) cause different integrin conformations, binding either to MAdCAM or VCAM (452). A explanation how the same integrin can select between different ligands was offered by Cormier and colleagues (92). They argued that besides $\alpha \mathrm{V} \beta 3$ integrin affinity for RGD, the accessibility of the ligand to the integrin binding pocket might be a regulating factor. Figure 3 highlights some of the headpiece features influencing integrin ligand binding selectivity, carrying the analysis also to laminin-binding integrins and how ligand accessibility and binding can be enhanced by the integrin headpiece movement. More detailed research will be required to challenge the notion of RGD ligand promiscuity and to show how switching between selective and promiscuous ligand binding can be of physiological relevance in vivo.

Given the extensive literature about mechanosensing and mechanotransduction by integrin mediated adhesions, it is almost surprising that the experimental data about the influence of mechanical forces on the integrin structure is rather limited. Based on molecular dynamics simulations of $\alpha \operatorname{IIb} \beta 3$ (500) and $\alpha \mathrm{V} \beta 3$ integrin (347) it was hypothesized that mechanical load on the $\beta 3$-subunit facilitates the headpiece opening of the integrin by increasing the hybrid domain swing-out. Therefore, one might argue that mechanical forces activate integrins, an exciting concept contributing to the emerging field of mechanobiology. So far, this idea is supported by experimental data for $\alpha \mathrm{L} \beta 2$ integrin (LFA1) $(292,313)$ and $\alpha \mathrm{V} \beta 3$ integrin (31, $83,146)$. In line with this, $\beta$-integrin subunits are especially well suited to bear mechanical load due to a reinforcement with two polypeptide chains (between the $\beta$-I-like and hybrid domain) or a disulfide bridge in addition to a polypeptide chain between their domains (113). Domainconnections in the $\alpha$-subunit miss these additional reinforcements, and the $\alpha$ integrin subunit may therefore unfold more easily under mechanical load.

\section{Similarities and differences between integrins}

Many cell culture studies compare $\alpha \mathrm{V} \beta 3$ integrin and $\alpha 5 \beta 1$ integrin $(34,69,98,369,388)$. Both belong to the group of RGD integrins, bind fibronectin and are expressed in both fibroblasts and endothelial cells. Accordingly, the overall structural organization is very similar. Nevertheless, there are important structural and functional differences between $\alpha \mathrm{V} \beta 3$ integrin and $\alpha 5 \beta 1$ integrin. In a recent study, Takagi and coworkers detected $\alpha \mathrm{V} \beta 3$ integrin to be present in the bent, extended-closed, and extended-open conformation in the absence of ligands or 
stabilizing antibodies (289). However, under identical conditions the authors failed to detect an extended-open conformation for $\alpha 5 \beta 1$ integrin. In contrast, the group of Timothy Springer detected all three conformations for $\alpha 5 \beta 1$ integrins by complexing them with conformationspecific antibodies (417). This approach also allowed them to measure affinities of specific conformations for RGD and fibronectin fragments (257). Interestingly, they detected a 4,000to 6,000-fold increase in affinity of the extended-open compared to the extended-closed conformation for cyclic RGD (cRGD) and fibronectin fragments. This is in clear contrast to $\alpha \operatorname{IIb} \beta 3$ integrin, for which an only 200-fold increase was reported (501). This difference in affinity increase during headpiece opening could imply $\alpha 5 \beta 1$ integrin to be 'locked' to its ligand when reaching the extended-open conformation. Such a strong binding to fibronectin could have evolved to support the mechanical stretching of the ligand during fibronectin fibrillogenesis (390), which is likely to be a non-linear and visco-elastic process, in which a rapid loss of tensional load in fibronectin fibrils should not result in the immediate dissociation from the integrin receptor. On the other hand, the evolution of a synergy site in fibronectin, specifically enhancing the on-rate for $\alpha 5 \beta 1$ integrin binding, may help to diversify the specific features of certain integrin/ligand pairs (302). At the same time, a strong binding with a low off-rate might also set the need for precise regulation of the activity of $\beta 1$ integrins by inhibitors (54) or by posttranslational modifications like phosphorylation, glycosylation, or acetylation. This example emphasizes the connection of structural differences and specific physiological tasks of $\alpha 5 \beta 1$ integrin in fibronectin fibrillogenesis. At the same time, it highlights the difficulties of generalizing concepts from well-studied integrins to the entire family of integrin receptors.

As mentioned above, collagen-binding integrins and leukocyte specific integrins differ from all other integrins by the presence of an $\alpha \mathrm{I}$ domain in the $\alpha$-subunit. Importantly, only this $\alpha \mathrm{I}$ domain binds the respective ligand, in contrast to a combined ligand binding by both subunits in integrins without $\alpha \mathrm{I}$ domain. This might explain why RGD-binding integrins, lacking an $\alpha \mathrm{I}$ domain, evolved a bigger variety of $\alpha$ - and $\beta$-subunit pairings (Figure 1). Interestingly, the initial binding pocket formed by the propeller domain in the $\alpha$-subunit and the $\beta$-I-like domain in the $\beta$-subunit is still present in $\alpha \mathrm{I}$ domain integrins. However, it is used by the $\alpha \mathrm{I}$ domain as an intramolecular 'pseudo-ligand' for recognition of the IEGT-peptide motif (Figure 3D). Additionally, $\alpha$ I domains have no ADMIDAS site, and their $\alpha$ I helix is always straightened during activation. Thus, ADMIDAS movement towards the ligand and $\alpha 1$-helix straightening during integrin activation might be used to fine-tune the affinity of the MIDAS site in $\beta$-I-like 
domains (495, 501). Therefore, Zhang and colleagues (495) argued that $\alpha \mathrm{I}$-domain integrins, missing this fine-tuning, might be better suited for fast on/off switching than integrins without $\alpha \mathrm{I}$ domain.

Another surprising mechanobiological feature of integrins are catch bonds between ligands and integrins, meaning that the lifetime of a bond increases when force is applied (82). As summarized by Cheng Zhu and colleagues (82), catch bonds are now described for $\alpha 5 \beta 1$ fibronectin, $\alpha \mathrm{V} \beta 3$ - fibronectin, $\alpha \mathrm{L} \beta 2$ - ICAM-1, $\alpha 4 \beta 1$ - VCAM-1, and $\alpha \mathrm{M} \beta 2$ - ICAM-1. As these authors point out, it is more appropriate to describe these bonds as catch-slip bonds, since the bond will change from a catch bond to a slip bond when the force on the bond exceeds a certain level. Catch bonds might have evolved to stabilize cell-ECM anchorage by allowing integrin-ligand bonds to persist under mechanical load, especially when the other bonds in their surrounding break by mechanical stress. Interestingly, catch bonds are documented also for other receptor-ligand pairs than integrins (Notch-Jagged1, VWF-GPIb $\alpha$, TCR-pMHC as described by Cheng Zhu and colleagues (82); E-Cadherins (356), P-Selectin-PSGL-1 (281)), as well as intracellular force-bearing connections like vinculin and actin (197). Potentially, catch bonds will emerge as the rule and not the exception whenever mechanical forces are involved in receptor ligand interactions. Still, the structural implementation of this feature within the integrin headpiece requires yet to be shown. The increasing unmasking of the positive charge of the metal ion at the MIDAS position and the consequentially tighter binding of the negatively charged Asp in the RGD peptide during integrin activation are, however, a plausible mechanism $(458,459)$ (Figure 2, 3). Catch bonds in $\alpha \mathrm{I}$ domain integrins $\alpha \mathrm{L} \beta 2$ and $\alpha \mathrm{M} \beta 2$ have to include the $\alpha$ I domain, but mechanisms in the $\beta$-I-like and hybrid domain could be analogous in integrins without $\alpha \mathrm{I}$ domain (82).

The in vivo importance of catch bonds might be best documented in the vasculature, where selectin-based catch bonds regulate leukocyte rolling in presence of shear stress caused by the blood stream (139). Additionally, recent examples of circulating tumor cells arresting in a $\beta 1$ dependent manner in the blood flow might indicate the relevance of catch bonds (281).

On a first glance, the structural understanding of integrins might appear quite detailed already. However, as described here, not every integrin is studied to the same extent, and the generalization of individual integrin qualities to other integrins might be misleading. While structural features of integrins can be linked to physiological settings, it is also clear that we are 
limited by techniques that allow us to test these hypotheses in vivo. Additionally, the examples of mechanical integrin regulation suggest that the transfer of data from experiments in the absence of force (in vitro studies, flow cytometry) to the in vivo setting is not always straightforward. Having said this, we are nevertheless convinced that the detailed understanding of even a few integrins will be useful as a framework to compare with other integrins, deducing their function based on differences and similarities.

\section{Chapter 3: The physiological role of integrin-dependent cell adhesion explained through several examples}

\section{Integrin affinity modulation versus clustering in the plasma membrane (talin and kindlin)}

When integrins recognize extracellular ligands and change from a low to a high affinity conformation, either by an outside-in or inside-out triggered mechanisms, they also start to form clusters in the membrane that are visible by light microscopy $(35,90,336)$. Using superresolution light microscopy, the initial formation of nano-clusters of 50 to 100 ligand-bound integrins can be detected (75), that will further assemble into larger integrin clusters to enable cell adhesion. The mechanistic connection between conformational activation of $\beta 3$-integrins and integrin clustering is still not fully understood, but requires at least extracellular ligandbinding, talin-head/integrin interaction and talin and kindlin binding to phosphoinositol lipids in the plasma membrane $(51,90)$.

Although $\alpha \operatorname{IIb} \beta 3$ and $\alpha v \beta 3$-integrin activation and clustering are among the best studied integrin processes, it is still not clear, why in resting platelets $\alpha 2 \beta 1$ integrin is in an apparently extended, ligand binding-competent, but not fully activated state $(289,312)$, while at the same time $\alpha \operatorname{IIb} \beta 3$ receptors are thought to be present in the platelet membrane in a bent-closed conformation (485). Differences between $\beta 1$ and $\beta 3$-integrins in the transmembrane and cytoplasmic $\alpha$-domain association, also known as the inner membrane clasp (Figure 2), could account for these different integrin resting states (271). Similarly, intracellular isoformselective integrin inhibitors could be responsible for maintaining distinct conformational pools of cell surface integrins, e.g. keeping $\alpha \operatorname{Ilb} \beta 3$ in a bent-closed conformation and preventing it from binding plasma fibrinogen, while presenting $\alpha 2 \beta 1$ in an extended conformation able to bind to exposed collagen fibers at sites of vessel damage (441). Support for the model of conformational activation of $\alpha \operatorname{IIb} \beta 3$ integrin has come mainly from the discovery of a ligandmimetic IgM monoclonal antibody (PAC-1) binding $\alpha \operatorname{IIb} \beta 3$ integrin on activated, but not 
resting platelets (394). Interestingly PAC-1 exhibits an RGD-related KYD sequence in the H3 loop of the heavy chain, thought to be responsible for $\alpha \operatorname{IIb} \beta 3$ binding. However, a report by Tomiyama and coworkers described two different IgG antibodies with the same KYD sequence that bound equally well to resting as well as activated platelets (439). Although this discrepancy in $\alpha \operatorname{Ilb} \beta 3$ binding by IgG and IgM antibodies can be explained by a specific conformation of the KYD-containing loop, probing $\alpha \mathrm{IIb} \beta 3$ integrin binding with Fab fragments of the PAC-1 antibody did not allow to discriminate between integrins on resting or talin-head activated platelets or CHO cells (62). Thus it appears possible that the large size of the PAC-1 IgM prevents it from efficiently recognizing the bent-closed $\alpha \operatorname{IIb} \beta 3$ integrin receptor. On the other hand it is also likely that the enhanced cell surface binding of PAC-1, e.g. observed during talinhead mediated $\alpha \operatorname{IIb} \beta 3$ activation (425), is due to talin-mediated $(90,380)$ or kindlin-induced integrin clustering (486). Such an increase in integrin clustering is particularly well detected due to the polyvalency of the PAC-1 ligand (62), therefore proposing that physiological insideout activation of the $\beta 3$-integrin receptor involves conformational changes of the integrin ectodomain as well as adapter-induced clustering of the receptors in the plasma membrane (90, 380). Kindlin appears to contribute to integrin clustering rather than to activation, co-operating with talin in this process (486).

The conformational activation of integrins has also been analyzed by a genetic screening approach based on a monovalent integrin ligand binding to the Drosophila $\alpha \mathrm{PS} 2 \beta \mathrm{PS}$ integrin. This study revealed mostly gain of function mutants in $\beta$ PS, stressing the physiological importance of keeping integrins in a low ligand-binding affinity state. On the other hand, the mutation of the juxtamembrane $\underline{\mathbf{C G F}} \underline{\mathbf{F}} \underline{\mathbf{R}}$ sequence in $\alpha \mathrm{PS} 2$ to CGFAN$\underline{\mathbf{A}}$ enhanced ligand

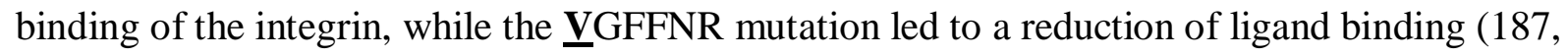
220). Interestingly, the mutated cysteine residue is conserved in $\alpha 3, \alpha 6, \alpha 8$ and $\alpha \mathrm{E}$-integrins (Figure 1) and known to be palmitoylated in $\alpha 3$ and $\alpha 6$-integrins (480), proposing the existence of still undiscovered mechanisms to control the integrin affinity state in general (such as kindlin) or in integrin-specific situations, such as in $\alpha$ PS2.

\section{The $\alpha I I b \beta 3$ receptor on platelets}

One of the best studied integrin structure-function relationship concerns the $\alpha \operatorname{IIb} \beta 3$ receptor expressed on platelets. Blood is coagulating through activation of platelets, that are stimulated by agonists such as ADP or thrombin, or by binding to injury-released, collagen-bound von 
Willebrand factor, leading to a conformationally induced change in the affinity of $\alpha$ IIb $\beta 3$ integrin (also known as GPIIb/IIIa) for circulating fibrinogen in the plasma (470). Based on this physiological example, the signal-mediated conformational change of $\alpha \operatorname{Ilb} \beta 3$ integrin and the subsequent binding of extracellular fibrinogen allowed to establish the concepts of insideout and outside-in signaling. The activation of $\alpha \operatorname{IIb} \beta 3$ integrin has to be strictly regulated to avoid a fatal thrombosis, therefore it cannot be activated by the always-present ligand fibrinogen. Instead, intracellular signals are required for $\alpha \operatorname{Ilb} \beta 3$ integrin activation, leading to fibrinogen binding and formation of a blood clot (i.e. inside-out signaling). These activating signals for $\alpha \operatorname{IIb} \beta 3$ integrin, on the other hand, have to originate from the outside, where a signal conveying the presence of a wound to the platelet triggers the intracellular cascade leading to $\alpha \operatorname{IIb} \beta 3$ integrin activation (i.e. outside-in signaling). Platelets express the collagen receptors GPVI and $\alpha 2 \beta 1$ integrin, both potentially sensing wound-exposed collagen, but the precise contribution of both receptors to $\alpha \operatorname{IIb} \beta 3$ integrin activation appears controversial $(279,309)$. Recent structural studies for $\beta 1$ integrins in the presence and absence of ligands revealed interesting differences to $\beta 3$ integrins with consequences for the structure-function relationship of both integrins. Takagi and coworkers found $\beta 1$ integrins in the absence of ligands to be mostly present in the extended-closed conformation, irrespective of the ion conditions (289). The same study, but also work by the group of Timothy Springer (417), detected extended-open conformation for $\beta 1$ integrins in the presence of ligands (or stabilizing antibodies). On the other side, $\beta 3$ integrins conformations were strongly affected by ion conditions, revealing conformations from bent, extended-closed to extended-open. Thus, $\beta 3$ integrins might be more susceptible to allosteric regulation by cytoplasmic adapters, while $\beta 1$ integrins are mostly regulated by the presence of ligands.

The inside-out activation of $\alpha \mathrm{IIb} \beta 3$ integrin is still a matter of research, but essential features include the activation of the Rap-1 GTPase, binding the talin rod-domain to release talin autoinhibition and to induce a mechanical coupling between the actin cytoskeleton (talin rod domain) and the integrin-cytoplasmic tail (talin head domain) $(64,230,416,454)$. Since the talin-integrin connection provided an explanation of the $\alpha \mathrm{IIb} \beta 3$ integrin activation mechanism, critical roles for additional integrin activators were not considered at the time. However, it has become clear, that the talin-head interaction with the cytoplasmic tail of the $\beta 3$-integrin receptor alone is not sufficient, and that the plasma membrane-associated adapter protein kindlin is at least equally, if not even more important than talin to induce $\alpha \operatorname{IIb} \beta 3$ integrin conformational 
activation and fibrinogen binding, subsequently triggering platelet and cell spreading $(295,437)$ (Figure 2, 3).

Several publications indicated Rap1-mediated activation of integrins to include the binding of RIAM to talin, as demonstrated for $\alpha \operatorname{IIb} \beta 3$ integrin (179). Recent publications analyzed this process in more detail and found RIAM-mediated activation to be specific for $\beta 2$ integrins, whereas within the same leukocytes $\alpha 4 \beta 1$ integrin is activated in a RIAM-independent manner (230, 414). Additionally, RIAM knockout mice showed no severe phenotype and unaltered $\beta 1$ and $\beta 3$ integrin activation $(230,414)$. Thus, it appears that pathways upstream of talin (and kindlin) are able to target and activate specific integrin subunits, enabling cells to react differentially to separate outside-in signals. One of these pathways may involve a direct activation of the talin-head domain by Rap1-binding, instead of an indirect, RIAM-dependent mechanisms $(58,68,502)$.

\section{The role of integrins in extracellular matrix assembly: fibronectin}

So far we have mainly considered the role of integrin receptors in a cell-autonomous way, as integrins are critical for cell anchorage to the extracellular matrix, providing signals for survival and proliferation. However, integrin receptors are also used by cells to organize or remodel the extracellular matrix. For example, cultured fibroblasts synthesize extracellular matrix proteins such as fibronectin, which they incorporate into an extracellular scaffold that allows their adhesion and generates survival signaling. In the well-studied case of fibroblasts cultured on fibronectin, the $\alpha v \beta 3$ integrin receptor assures the binding of the cell periphery to the culture substrate, while $\alpha 5 \beta 1$ is "spinning" or "weaving" a fibronectin network around the center of the spread cells by forming fibrillar adhesions (324). In a preformed 3D fiber network the classical distinction between focal and fibrillar adhesion is no longer maintained $(95,473)$. As mentioned in Chapter 1, transformed fibroblasts loose the capacity to synthesize fibronectin fibrils. In cancer tissues cancer-associated fibroblasts partially compensate this by excessive deposition of extracellular matrix in the tumor stroma (CAFs) (126, 316). Interestingly, the enhanced deposition of extracellular matrix by CAFs should be taken into consideration during the treatment of tumor patients, as the enhanced stiffness of the tumor stroma induces survival signaling in B-RAF inhibitor-treated melanoma cells (191). The mechanisms responsible for fibronectin fibril synthesis are still incompletely understood, but involve the cytoplasmic integrin adapter protein tensin1 (324). Interestingly tensin1 function is targeted also by 
intracellular metabolic pathways, linking integrin-dependent fibronectin assembly to the level of glucose in the tissue and in general to the metabolic state of a cell in a tissue $(157,288)$. Moreover, the tracking of fluorescent $\beta 1$-integrin in astrocytes has allowed to connect the assembly of fibronectin fibers in fibrillar adhesions to the simultaneous association of GFPlabeled VEGF with such newly synthesized fibronectin fibers (119). These results do not only provide a unique insight into the process of integrin-dependent fibronectin assembly, but also highlight the fact that the extracellular matrix is providing a delicately tensioned scaffold, binding and storing growth factors and releasing this pool of signaling molecules in the case of tissue injury or pathological signaling in the case of fibrosis (see Chapter 9). Rather recently, it became evident that not only tensins, but also proteins from the kank family are relevant in fibrillogenesis (420). Kank2 reduces the affinity of the talin rod for actin, thereby weakening the mechanical load on the ECM-integrin-actin axis. This process acts in parallel to the maturation of focal adhesions to fibrillar adhesions and their translocation to the cell center. It might be counterintuitive that mechanical alignment of fibronectin fibers is mediated by fibrillar adhesions under low mechanical load. Interestingly, detailed studies with atomic force microscopy revealed that the initial reorganization of fibronectin fibers already occurs in the cell periphery, where integrins are under higher mechanical load (174). Thus, we envision a model of initial fibronectin stretching in the cell periphery, including higher forces on the integrin-fibronectin link. After this opening of cryptic binding sites on fibronectin, and potentially detachment from the substrate, small fibrils are aligned and organized to form bigger fibrils. This translocation of detached fibrils might benefit from high-affinity binding even under low force, which is achieved by $\alpha 5 \beta 1$, but not by $\alpha \mathrm{V} \beta 3$ integrin $(31,388)$, while kank2 orchestrates the change in force level through the modulation of the talin-actin connection. Interestingly, kank2 might also be important for the effect of microtubules and focal adhesion stability (77). Kank binds simultaneously to the CLASP family of microtubules plus-end binding proteins, the R7 subdomain of talin, as well as the membrane-bound liprin/LL5 scaffold, which functionally associates focal adhesions with the vesicular transport machinery $(53,410)$.

The role of integrins in extracellular matrix assembly: laminin and collagen

Collagen and fibronectin are both major components of the ECM, responsible for the structural organization and mechanical integrity of the ECM. Collagen type I is a prime example for fibrillar collagens, in contrast to collagen type IV that forms networks in the basement 
membrane. Four integrins, $\alpha 1 \beta 1, \alpha 2 \beta 1, \alpha 10 \beta 1$, and $\alpha 11 \beta 1$ (all containing an $\alpha$-I domain; see Figure 1), are reported to bind collagens with certain preferences for either collagen I or collagen IV (222). Both collagens also have different mechanisms leading to their structural arrangement in the ECM. Collagen I is known to align with fibronectin and to gradually replace it in the ECM during wound healing (287). Interestingly, collagen I preferentially binds to relaxed fibronectin fibers (236). On the other hand, the same study (236) showed fibronectin fibers to be under increased stress in the absence of collagen I, thereby emphasizing the relevance of collagen for the mechanical state of ECM. A self-assembly of fibrillar collagen, used for surface coatings in cell culture studies, seems to be much less relevant in vivo (215).

The experimental observation of the basement membrane organization and its main components collagen IV, laminin, nidogen, and perlecan in epithelial cells is more complex. Collagen IV was shown to be dispensable for the initial organization of the basement membrane in the embryo (before E10 in mice), but to be essential in later developmental stages (341). Thus, like fibrillar collagen, also collagen IV is highly important for the structural integrity of the ECM. It is well accepted that in basement membranes $\alpha 3 \beta 1, \alpha 6 \beta 1$ and $\alpha 6 \beta 4$ integrins contribute to adhesion of epithelial cells by recognizing the c-terminal globular domains of the laminin $\alpha$ subunit (see also Figure 3D) (483). In the absence of these integrins, the epithelia detach and blisters form $(111,112)$. Defects in the deposition and organization of such basement membranes have been rarely reported, but it has been recognized that laminin binding integrins

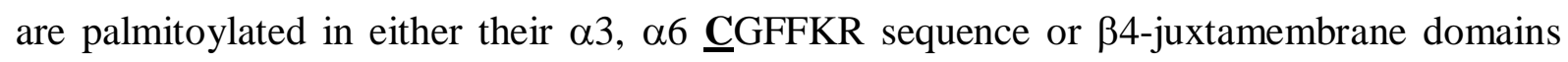
(480). The absence of this reversible lipidation affects laminin-dependent adhesion and association with the palmitoylated tetraspanins in the plasma membrane $(39,479)$. Interestingly, the depletion of the tetraspanin CD151 causes kidney failure associated with altered glomerular basement membranes (378). Moreover, in tissue culture $\alpha 3 \beta 1$-integrins showed enhanced, focal adhesion-like clustering due to the absence of the tetraspanin CD151 (377), suggesting that membrane distribution and tetraspanin association of laminin-binding integrins are not only regulating the adhesion to basement membranes, but also their assembly.

\section{Non-classical integrin mediated adhesions}

Integrin-mediated adhesions were often classified according to a maturation sequence starting from nascent adhesions, leading over focal complexes and focal adhesions to fibrillar adhesions $(88,151)$. However, it is also clear that not all integrin adhesions follow this scheme. 
Podosomes and invadopodia (now summarized as invadosomes) were already described in the 1980s (reviewed in $(195,300)$ ), and their structural organization differs drastically from 'classical' integrin adhesions. Invadosomes have a central actin core oriented perpendicular to the substrate and surrounded by a belt of adhesome proteins like talin and vinculin. As the name indicates, invadosomes are involved in ECM degradation, thereby supporting invasion of the cell into the degraded, softened tissue. This is achieved by the delivery of matrix metalloproteases (MMPs) to sites of invadosomes, where they are secreted and digest the ECM (338). This process was shown to also occur at focal adhesions (410), but appears to be more prominent at invadosomes. For more insights about integrin recycling and endo- and exocytosis at sites of integrin adhesions we would like to refer to excellent reviews about this topic (142, 293, 328).

More recently, a new type of adhesions specific for $\alpha \mathrm{V} \beta 5$ integrin emerged (270). During the analysis of integrin adhesions throughout the cell cycle the authors detected an enrichment of $\beta 5$ integrin to specific adhesion structures during interphase. Interestingly, $\alpha \mathrm{V} \beta 5$ integrinmediated adhesions in these cells recruited no classical adhesome proteins like talin1, kindlin2 or vinculin and were not coupled to actin filaments. Additionally, their shape differed from classical adhesions; they formed a dense net of adhesive structures coined reticular adhesions. The reticular adhesions recruited adapters of clathrin-mediated endocytosis, potentially contributing to their ability to stay attached to the matrix during mitosis and to serve as a 'adhesion memory' during re-spreading after mitosis. Additional studies by other groups confirmed this dependence of $\alpha \mathrm{V} \beta 5$ integrin-mediated adhesions on adapters of clathrinmediated endocytosis, in contrast to classical adhesome proteins $(38,503)$. Interestingly, $\alpha \mathrm{V} \beta 5$ integrin adhesions associated with clathrin adapters have a capacity for mechanosensing and mediate cell adhesion even in the absence of the classical adhesion machinery. $\beta 5$ integrin knockout mice develop age-related retinal dysfunction due to the lack of $\beta 5$ integrin-dependent phagocytosis of photoreceptors by retinal pigment epithelial cells (304). The relation of this finding to reticular adhesions in cell culture experiments remains to be shown in future experiments.

\section{Forces in tissues}

The third phase of integrin research, reconciling known features of integrins with their ability of mechanosensing and -transduction, is presumably just beginning. But can we expect that these findings have a relevance in more physiological settings, compared to cells cultured on 
glass and plastic coated surfaces? We believe that recent findings strongly suggest important roles of mechanical parameters (e.g. tissue stiffness, ligand geometry, elasticity vs. viscoelasticity) in developmental and pathological settings. As we discuss in Chapters 6 and 9, integrin-mediated mechanotransduction follows a sigmoidal mechanoswitch triggered around $5 \mathrm{kPa}$ substrate stiffness. It is striking that most healthy tissues have a stiffness below this point, while fibrotic tissue is stiffer than $5 \mathrm{kPa}$ (see Chapter 9). At the same time, stiffness gradients observed during the development of Xenopus (438) and Drosophila (94) make clear that developing organisms consist of regions with distinct mechanical properties. Richard Harland and coauthors showed in elegant experiments that the positioning of feathers in developing chicken skin is based on mechanical signals (401). Therefore, it will be not surprising when more reports uncover the contribution of integrin mechanosensing and -transduction in development and pathologies. On a more structural level, it is interesting to note that both talin (265) and integrins (422) are aligned with the force vector of actomyosin forces. As mentioned in Chapter 2, MD simulations suggest that forces parallel to the membrane (imitating retrograde actin flow) support the extended-open conformation of $\alpha \operatorname{IIb} \beta 3$ integrin, while the extendedclosed conformation is stabilized by forces perpendicular to the membrane $(422,500)$. Additionally, work in Drosophila indicates that integrins and talins might experience unique force vectors in different tissues (229). Combined with the findings that specific integrin conformations bind ligands selectively (see above), differential force vectors in tissues might be a mechanism to tune the physiological needs for integrin activation and signaling. This very likely includes also mechanical regulation of integrin adapter conformations (208). However, the testing of these hypotheses will require improved tools to measure forces and force vectors in vivo. Several studies in Drosophila offered interesting insights into this question and might indicate a renaissance for this model organism $(166,172,229,249,431)$.

\section{Chapter 4: Regulation of integrins by adapter proteins}

Integrins recruit hundreds or even up to thousand different proteins, building the so-called adhesome $(63,239,387)$. However, a recent meta-analysis defined a consensus adhesome of 60 proteins (194), that the authors organized in four groups: 1) ILK - PINCH - kindlin, 2) FAK - paxillin, 3) talin - vinculin and 4) $\alpha$-actinin - zyxin - VASP. Most of these proteins have been mapped into functional layers with super-resolution imaging (217). The importance of these sets of proteins is reflected by their frequent discussion in reviews on integrin-mediated adhesions (199, 207, 208, 364, 371, 419). 
For this review, we wanted to focus on adapters that directly bind integrins. Therefore, we curated a list of such direct integrin adapters (Table 1). Some of these proteins, like talin or FAK, are well known in the integrin field, others are less well studied and their effect on integrins might not be fully established yet. Additionally, the large diversity of the integrin family as well as their extensive functional diversity suggests that integrins are regulated in a cell- and integrin-type specific manner. For example, kindlin3 is only expressed in hematopoietic cells (49) but kindlin1 and kindlin2 show unique interactions with integrins in keratinocytes (36), indicating that they are not functionally redundant (371). Talin1 and talin2 are shown to influence mechanotransduction differently (26) and to possess altered affinity for the $\beta 1$ - and $\beta 3$-integrin subunits (15). They also differ in their expression within tissues, with e.g. talin 2 being the dominating form in striated muscle (392) and required for fibronectin assembly (345). Nevertheless, the knockout of talin1 is embryonic lethal, while talin2 knockout mice show a dystrophic phenotype (104). We assume that further detailed and isoform-specific analysis will reveal more selective integrin-adapter interactions and their evolution for specific physiological needs.

To support a conceptual understanding of integrins we want to introduce 5 functions that are mediated by integrin adapters: (i) activation, (ii) inactivation, (iii) inhibition, (iv) signaling, and (v) mechanosensing. We expect that less-studied adapters can be explained within the framework of these functions. This classification also implies that adapters can have more than one function.

\section{Activation}

Talin and kindlin activate integrins (= change the extracellular conformation) and increase their affinity for ligands in a process of inside-out activation involving interaction of these adapter proteins with the $\beta$-integrin cytoplasmic tail. Both talin and kindlin are required for integrin activation and clustering, but appear to differentially contribute to mechanosensing (talin) and signaling (kindlin) $(354,437)$. An important part of integrin activation are the unclasping of the $\alpha$ - and $\beta$-subunit at the level of the transmembrane and cytoplasmic tails as well as the physical connection to the actin cytoskeleton. The $\alpha$-integrin cytoplasmic tails vary in sequence and length, but share a common GFFKR motif partially buried within the cell membrane interacting with the transmembrane domain of the $\beta$-subunit (Figure 4 and 7). The cytoplasmic tails of 
$\beta$-integrins contain two conserved PTB (phosphotyrosine-binding domain) binding sequences (Figure 7): the membrane-proximal $\mathrm{NPx}(\mathrm{Y} / \mathrm{F})$ and membrane-distal $\mathrm{Nxx}(\mathrm{Y} / \mathrm{F})$ motifs. Talin binds both the membrane-proximal helix and the $\beta$-integrin tail up to the first, membraneproximal $\mathrm{NPx}(\mathrm{Y} / \mathrm{F})$ motif $(16,150,457)$. Kindlin binds to the inter-NXXY-region and the membrane-distal Nxx(Y/F) motif (253). Talin and kindlin can thus bind integrin simultaneously (46). Furthermore, the binding of paxillin to kindlin has been found to promote integrin activation (149), potentially further increasing the complexity of the integrin activating intracellular adapter complex. As shown for some integrins, outside-in activation is triggered by ligand binding, and therefore also ligands can be considered as integrin activators. Additionally, mechanical load supports integrin activation (see Chapter 2) and could therefore be considered as an activator. In this context, it is important to note that the F-actin linkage to integrins is the mechanically weak point, where integrin clustering, recruitment of adapter proteins (such as vinculin) and regulation of actin (de)polymerization are likely to be involved $(200,327)$.

\section{Inactivation}

Integrin inactivators ensure the dynamic regulation of cell adhesion, e.g. by unbinding from areas a cell wants to avoid, allowing migration away from this location. Phosphorylation by kinases, most notably FAK and Src, increases the turnover of integrins and integrin-mediated adhesions. Src serves as an integrator of several pathways, as it was shown that local ephrin/Eph signaling influences integrin-mediated adhesions in its vicinity via a Src-FAK-paxillin cascade (84). Additionally, endocytosis allows integrin detachment from the ECM and thereby inactivates integrins: Dab2/clathrin-mediated endocytosis was shown to replace integrin activators like talin and kindlin from $\beta 3$ integrin and to mediate integrin endocytosis (488). Interestingly, Dab2-mediated endocytosis was increased in the absence of mechanical load on integrins, indicating that a lack of force can participate in integrin inactivation. Thus, there might be different ways integrins can be inactivated, involving either an inside-out mechanisms, e.g. proteolytic degradation of adapter proteins (386), or phosphorylation of integrins or adapters (as review, see (148)). On the other hand, the proteolytic degradation of extracellular matrix generates protein fragments with intact integrin binding functions. Such ECM fragments, also termed matrikines, can bind to integrins in their soluble forms, maintaining the extended-open conformations of integrins without mechanical linkage to the ECM. At this point, it is not clear why such a tension-free state would enhance the exchange of talin with 
other intracellular adapters, but it leads to the subsequent internalization of the complex, as observed for fibronectin-bound receptors $(76,269)$.

As just mentioned, an interesting aspect of integrin activation vs. inactivation is the role of force in these processes. Why are activators and inactivators needed, if increased mechanical load activates integrins and decreased mechanical load inactivates them? First of all, it is important to keep in mind that force needs a physical link to be transmitted: there is no relevant mechanical force on integrins without talin-mediated actin linkage (354). Maybe even more importantly, integrins are not purely mechanical anchors, but also measure tension, create and integrate biochemical signals that in turn will change cell adhesion, motility and proliferation. These different integrin functions should be reflected by different modes of integrin (in)activation. At the same time, the crosstalk between different modes of integrin activation would allow to integrate mechanical and biochemical signals at the level of cell adhesion.

\section{Inhibition}

Some integrins are found in the membrane in an inactive, bent conformation (see Chapter 2). Additionally, the pool of inactive integrins can be stabilized or increased by integrin inhibitors (summarized in (54)). ICAP, for example, binds to the tail of $\beta 1$ integrin and prevents activation (61), while filamin A is shown to inhibit integrin activation by establishing a ternary complex with $\alpha I I b$ and $\beta 3$ integrin subunits, preventing the separation of the integrin subunits (264) (see Figure 3E).

\section{Signaling and Mechanosensing}

Finally, some adapters of integrins are involved in signaling or mechanosensing. Signaling adapters include kinases like FAK, but also paxillin, that serves as a dynamic scaffold recruiting different GEFs and GAPs, thereby regulating Rho-GTPases signaling and the organization of the actin cytoskeleton (103). Mechanosensing adapters, on the other hand, include e.g. the Srcsubstrate p130Cas, that is phosphorylated upon cell stretching (385). The ability of adapters to sense and transduce mechanical signals is often coupled to force-mediated conformational changes (208). Talin is an example, having several cryptic vinculin- and hidden actin-binding sites that become accessible when the talin rod domain is put under tension $(24,353,365)$. In turn, the tension-exposed vinculin binding sites will enhance the physical anchorage of the talin rod to the actin cytoskeleton via newly recruited vinculin. The examples of FAK and talin 
illustrate that the same adapter can fulfill different tasks, according to the functional classification presented here.

\section{The slanted fence model of focal adhesions}

What can we say about the spatial organization of integrin adapters within focal adhesions? Super-resolution light microscopy has allowed the analysis of integrin-dependent adhesions in great details. This strategy involves on the one hand the analysis and tracking of individual integrin receptors in living cells, on the other the identification of the spatial organization of key adhesion components $(217,373)$. In fact, tracking of individual $\beta 3$ or $\beta 1$ integrins in living cells revealed the transient immobilization of these receptors within paxillin-positive focal adhesions $(373,456)$. Moreover, cytoplasmic adapter proteins such as talin were directly recruited from a cytoplasmic pool into focal adhesions, suggesting that the stabilization of the talin/integrin interaction seen e.g. during talin-induced integrin clustering occurs inside the adhesions themselves and not as a precursor outside of adhesions. The analysis of elongated focal adhesions by interferometric photoactivated localization microscopy (iPALM) has revealed specific membrane distances for different types of integrin adapter proteins. While the paxillin/FAK/src module is located in a membrane-proximal "signaling layer", the c-terminal F-actin binding region of talin is located distant from the membrane within the F-actin and vinculin cross-linking domain of focal adhesions. Moreover, the local tension induced on the talin-rod domain is directly reflected by the orientation of the F-actin network forming the backbone of adhesion (238). If these positional informations are integrated with the lateral Factin/myosin tension as well as the recent interactions of paxillin and DLC within the talin R8 bundle (491), one possible orientation is that of a slanted fence, similar to mobile fence systems used in the alpine regions. These slanted fences are stabilized by slanted long poles, representing the extended talin and F-actin fibers, and held in place by vertical poles, laterally connecting the fence structure (see for example https://de.wikipedia.org/wiki/Zaun\#/media/File:Schrankzaun2.JPG).

For focal adhesions, we are proposing that the flexible regions in paxillin and FAK could serve as dynamic connectors between the different layers of the focal adhesion, reacting to force changes in the lateral as well as vertical axis of the tethered talin-rod domain (Figure 6). As for the slanted fence, such a dense interaction in lateral and axial direction would increase the stability of the system against perturbations from multiple directions. 
Table 1 Integrin cytoplasmic adaptors and their known properties. The abbreviations of the detection methods are AC: affinity chromatography, B: biosensor assay, EA: enzyme assay, ELISA: enzyme-linked immunosorbent assay, IP:

immunoprecipitation, ITC: isothermal titration calorimetry, NMR: nuclear magnetic resonance spectroscopy, PD: pulldown assay, XRAY: X-ray crystallography, Y2H: yeast two-hybrid, O: other. The PDB code refers to the available structural information in the Protein Data Bank (42). IBS1 and IBS2: integrin binding sites 1 and 2 in talin. The list is not exhaustive.

\begin{tabular}{|c|c|c|c|c|c|c|}
\hline Adapter & Integrin & Approximate binding site or interaction & $\begin{array}{l}\text { Detec- } \\
\text { tion }\end{array}$ & $\begin{array}{l}\text { PDB } \\
\text { code }\end{array}$ & $\begin{array}{l}\text { Refe- } \\
\text { rence }\end{array}$ & $\begin{array}{l}\text { Proposed } \\
\text { role }\end{array}$ \\
\hline 12-LOX & $\beta 4$ & residues $661-1752$ & IP & & $(432)$ & $\begin{array}{l}\text { fatty acid } \\
\text { metabolism }\end{array}$ \\
\hline $14-3-3$ & $\begin{array}{l}\beta 2, \beta 1 \mathrm{~A}, \\
\alpha 4\end{array}$ & $\begin{array}{l}\text { T758-phosphorylated (by PKC) } \beta 2: \\
\text { KSA[pT]TTVMNP, } \alpha 4: \text { KRQYK[pS]IL }\end{array}$ & $\begin{array}{l}\text { AC, IP, } \\
\text { XRAY }\end{array}$ & $\begin{array}{l}2 \mathrm{~V} 7 \\
\mathrm{D}, \\
4 \mathrm{HK} \\
\mathrm{C}\end{array}$ & $\begin{array}{l}(48, \\
102, \\
129, \\
428)\end{array}$ & $\begin{array}{l}\text { integrin } \\
\text { activator (78) }\end{array}$ \\
\hline $4.1 \mathrm{~B}$ & $\beta 8$ & DYRVSASKKDKLILQSVCTRAVTYRREK & Y2H, IP & & $(283)$ & \\
\hline $4.1 \mathrm{G}$ & $\beta 1$ & & $\mathrm{IP}, \mathrm{PD}$ & & $(81)$ & \\
\hline $4.1 \mathrm{R}$ & $\beta 1$ & & IP, PD & & $(80)$ & \\
\hline Abl2 & $\beta 1$ & KFEKEKMNAK; phosphorylates Y783 & $\begin{array}{l}\text { IP, PD, } \\
\text { EA }\end{array}$ & & $\begin{array}{l}(404, \\
453)\end{array}$ & $\begin{array}{l}\text { tyrosine } \\
\text { kinase }\end{array}$ \\
\hline ACAP1 & $\beta 1$ & DRREFAKFEK & $\mathrm{PD}, \mathrm{IP}$ & & $\begin{array}{l}(32, \\
254)\end{array}$ & $\begin{array}{l}\text { integrin } \\
\text { recycling } \\
(254)\end{array}$ \\
\hline AKT1 & $\beta 3$ & phosphorylates T753 & EA & & $(227)$ & $\begin{array}{l}\text { Ser/Thr } \\
\text { kinase }\end{array}$ \\
\hline $\begin{array}{l}\text { Annexin } \\
\text { A5 }\end{array}$ & $\beta 5$ & FQSERSRARYEMAS & $\mathrm{O}$ & & $(70)$ & \\
\hline AP2M1 & $\alpha 4$ & QYKSILQE & XRAY & 5FPI & $(141)$ & $\begin{array}{l}\text { integrin } \\
\text { endocytosis } \\
(141)\end{array}$ \\
\hline AUP1 & $\alpha \mathrm{IIb}$ & KVGFFKR & $\begin{array}{l}\mathrm{Y} 2 \mathrm{H} \\
\mathrm{PD}\end{array}$ & & $(218)$ & $\begin{array}{l}\text { recruits SYK } \\
\text { to } \alpha I l b \text { tail } \\
(219)\end{array}$ \\
\hline BIN1 & $\alpha 3$ & KCGFFKR & $\mathrm{Y} 2 \mathrm{H}$ & & $(466)$ & \\
\hline CD98hc & $\begin{array}{l}\beta 1 \mathrm{~A}, \beta 3 \\
\text { not } \beta 1 \mathrm{D} \\
\text { or } \beta 7\end{array}$ & $\beta 1 \mathrm{~A}:$ NPKYEGK, $\beta 3:$ TNITYRGT & $\mathrm{AC}$ & & $\begin{array}{l}(346, \\
494)\end{array}$ & $\begin{array}{l}\text { promotes } \\
\text { integrin } \\
\text { signaling } \\
(346)\end{array}$ \\
\hline CENP-R & $\beta 3$ & $\beta 3$ : S752P weakens the binding & Y2H, IP & & $(395)$ & \\
\hline CIB1 & $\begin{array}{l}\alpha \mathrm{Ilb}, \\
\alpha \mathrm{V}, \alpha 5, \\
\alpha 2, \alpha 3, \\
\alpha 4, \alpha \mathrm{M}, \\
\alpha \mathrm{L}, \alpha 11\end{array}$ & aIlb: LVLAMWKVGFFKRNR & $\begin{array}{l}\text { Y2H, } \\
\text { IP, ITC, } \\
\text { O }\end{array}$ & & $\begin{array}{l}(37, \\
145, \\
303, \\
400, \\
475, \\
487)\end{array}$ & $\begin{array}{l}\text { inhibits } \\
\alpha \operatorname{Ilb} \beta 3 \\
\text { activation } \\
(490)\end{array}$ \\
\hline Csk & $\beta 3$ & & IP & & $(314)$ & Tyr kinase \\
\hline $\begin{array}{l}\text { Cytohesin- } \\
1\end{array}$ & $\beta 2$ & $\beta 2:$ WKALIHLSDLREYRRFE & $\begin{array}{l}\text { Y2H, } \\
\text { IP, PD, } \\
\mathrm{O}\end{array}$ & & $\begin{array}{l}(29, \\
154, \\
232, \\
363)\end{array}$ & $\begin{array}{l}\text { an Arf-GEF, } \\
\text { restrains } \\
\alpha \mathrm{M} \beta 2 \\
\text { activation } \\
(29), \\
\text { promotes } \\
\alpha 4 \beta 1 \text { and } \\
\alpha 5 \beta 1 \text { integrin } \\
\text { activation } \\
(28)\end{array}$ \\
\hline
\end{tabular}




\begin{tabular}{|c|c|c|c|c|c|c|}
\hline Dab1 & $\beta 1 \mathrm{~A}, \beta 3$ & membrane-distal NxxY & PD & & $(65)$ & $\begin{array}{l}\text { adaptor } \\
\text { protein }\end{array}$ \\
\hline Dab2 & $\beta 3, \beta 5$ & membrane-distal NxxY & PD & & $(65)$ & $\begin{array}{l}\text { adaptor } \\
\text { protein }\end{array}$ \\
\hline Dok1 & $\begin{array}{l}\beta 3, \beta 1 \mathrm{~A}, \\
\beta 7, \beta 2\end{array}$ & $\begin{array}{l}\text { phosphorylated NPxY ( } \beta 3, \beta 1 \mathrm{~A}, \beta 7) \text {, phosphorylated } \\
\text { NxxY }(\beta 3) \text {, S756-phosphorylated } \beta 2\end{array}$ & NMR & & $\begin{array}{l}(14, \\
175 \\
320)\end{array}$ & $\begin{array}{l}\text { adaptor } \\
\text { protein }\end{array}$ \\
\hline EED & $\begin{array}{l}\beta 7, \alpha 4, \\
\alpha \mathrm{E}\end{array}$ & $\beta 7:$ RLSVEIYDR & Y2H, IP & & $(363)$ & $\begin{array}{l}\text { polycomb } \\
\text { protein }\end{array}$ \\
\hline EIF6 & $\beta 4$ & $\begin{array}{l}\text { 1st and 2nd FNIII domains and the connecting } \\
\text { sequence }\end{array}$ & $\begin{array}{l}\mathrm{Y} 2 \mathrm{H} \\
\mathrm{PD}\end{array}$ & & $(44)$ & $\begin{array}{l}\text { ribosome } \\
\text { binding }\end{array}$ \\
\hline EPS8 & $\begin{array}{l}\beta 1 \mathrm{~A}, \beta 3 \\
\beta 5\end{array}$ & membrane-proximal NPxY & PD & & $(65)$ & \\
\hline Erbin & $\beta 4$ & $\begin{array}{l}\text { 4th FNIII domain and C-terminal sequence (res. 1457- } \\
\text { 1752) }\end{array}$ & $\begin{array}{l}\mathrm{Y} 2 \mathrm{H} \\
\mathrm{PD}\end{array}$ & & $(133)$ & \\
\hline Ezrin & $\beta 4$ & & IP, PD & & $(451)$ & $\begin{array}{l}\text { promotes } \beta 4 \\
\text { expression } \\
(451)\end{array}$ \\
\hline FAK1 & $\beta 5, \beta 3$ & $\begin{array}{l}\text { 33: complete cytoplasmic tail } \\
\beta 5+\text { Y861-phosphorylated FAK1: } \\
\text { QSERSRARYEMASNPLYRKPISTHTVDFTFNKFN } \\
\text { KSYNGTVD } \\
\beta 5+Y 861 \text {-nonphosphorylated FAK1: complete } \\
\text { cytoplasmic tail }\end{array}$ & IP, PD & & $(120)$ & Tyr kinase \\
\hline FAK2 & $\beta 3$ & $\beta 3:$ LYKEATSTFTNITYRGT & $\mathrm{IP}, \mathrm{O}$ & & (333) & Tyr kinase \\
\hline FHL2 & $\begin{array}{l}\alpha 3, \alpha 7 \\
\beta 1, \beta 2 \\
\beta 3, \beta 6\end{array}$ & $\begin{array}{l}\alpha 7 \mathrm{~A}: \text { AVQPSAMEAGGP, } \alpha 7 \mathrm{~B}: \\
\text { GTIQRSNWGNSQWEGS, } \beta 1 \text { A: VVNPKYEGK, } \\
\alpha 3: \text { ARTRALYEAKRQ }\end{array}$ & Y2H, IP & & $\begin{array}{l}(382, \\
465)\end{array}$ & $\begin{array}{l}\text { adaptor } \\
\text { protein }\end{array}$ \\
\hline FHL3 & $\begin{array}{l}\alpha 3, \alpha 7 \\
\alpha \mathrm{V}, \beta 1\end{array}$ & $\begin{array}{l}\alpha 7 A: \text { GTVGWDSSSGRST; } \alpha 7 \mathrm{~B}: \\
\text { DAHPILAADWHPELGP }\end{array}$ & Y2H, IP & & $(382)$ & $\begin{array}{l}\text { adaptor } \\
\text { protein }\end{array}$ \\
\hline FilaminA & $\begin{array}{l}\beta 1 \mathrm{~A}, \\
\beta 1 \mathrm{D}, \beta 3, \\
\beta 7, \beta 2 \\
\beta 6, \alpha \mathrm{IIb}\end{array}$ & $\begin{array}{l}\beta 2: \text { LFKSATTTVMN } \\
\beta 3: \text { PLYKEATSTFT } \\
\beta 7: \text { LYKSAITTTI } \\
\alpha I I b: \text { WKVGFFKRNRP }\end{array}$ & $\begin{array}{l}\text { NMR, } \\
\text { XRAY, } \\
\text { PD, } \\
\text { Y2H, B }\end{array}$ & $\begin{array}{l}2 \mathrm{MT} \\
\mathrm{P} \\
2 \mathrm{BR} \\
\mathrm{Q} \\
2 \mathrm{JF} 1\end{array}$ & $\begin{array}{l}(137 \\
211 \\
223 \\
264 \\
428)\end{array}$ & $\begin{array}{l}\text { actin binding; } \\
\text { competes } \\
\text { with talin } \\
(223)\end{array}$ \\
\hline FilaminB & $\begin{array}{l}\beta 1 \mathrm{~A}, \\
\beta 1 \mathrm{D}, \beta 3 \\
\beta 6\end{array}$ & & $\begin{array}{l}\mathrm{Y} 2 \mathrm{H} \\
\mathrm{PD}\end{array}$ & & $(137)$ & actin binding \\
\hline FilaminC & $\beta 1 \mathrm{~A}$ & & $\begin{array}{l}\mathrm{Y} 2 \mathrm{H} \\
\mathrm{PD}\end{array}$ & & $(164)$ & actin binding \\
\hline FRMD5 & $\beta 5$ & & PD, IP & & $(196)$ & $\begin{array}{l}\text { interacts with } \\
\text { and inhibits } \\
\text { ROCK1 } \\
\text { (196) }\end{array}$ \\
\hline Fyn & $\beta 3$ & & IP & & $\begin{array}{l}(18,19 \\
314)\end{array}$ & Tyr kinase \\
\hline GIPC1 & $\begin{array}{l}\alpha 3, \alpha 6 \\
\alpha 5\end{array}$ & $\alpha 3:$ ERLTSDA, $\alpha 6:$ NRNESYS & $\begin{array}{l}\mathrm{Y} 2 \mathrm{H}, \\
\mathrm{PD}\end{array}$ & & $(434)$ & \\
\hline HAX1 & $\beta 6$ & KLLVSFHDRKEVAKFEAERSKAKWQTGT & Y2H, IP & & $(357)$ & $\begin{array}{l}\text { clathrin- } \\
\text { mediated } \\
\text { endocytosis } \\
\text { of } \alpha \mathrm{V} \beta 6 \\
(357)\end{array}$ \\
\hline Hck & $\begin{array}{l}\beta 1, \beta 2, \\
\beta 3\end{array}$ & & IP & & $(18)$ & Tyr kinase \\
\hline
\end{tabular}




\begin{tabular}{|c|c|c|c|c|c|c|}
\hline ICAP1 & $\beta 1$ & NxxY; KSAVTTVVNP & $\begin{array}{l}\text { PD, } \\
\text { Y2H, } \\
\text { IP, } \\
\text { XRAY }\end{array}$ & $\begin{array}{l}4 \mathrm{DX} \\
9\end{array}$ & $\begin{array}{l}(74, \\
267 \\
498)\end{array}$ & $\begin{array}{l}\text { negative } \\
\text { regulator of } \\
\beta 1 \text { integrin, } \\
\text { competes } \\
\text { with talin } \\
\text { (55) }\end{array}$ \\
\hline ICln & $\alpha \mathrm{Ilb}$ & KVGFFKR & $\begin{array}{l}\mathrm{IP}, \mathrm{PD}, \\
\mathrm{B}, \mathrm{O}\end{array}$ & & $(241)$ & \\
\hline ILK & $\beta 1, \beta 3$ & & $\mathrm{Y} 2 \mathrm{H}, \mathrm{IP}$ & & $(181)$ & $\begin{array}{l}\text { (pseudo-) } \\
\text { kinase, part } \\
\text { of ILK- } \\
\text { PINCH- } \\
\text { Parvin } \\
\text { complex, } \\
\text { triggers F- } \\
\text { actin } \\
\text { bundling } \\
(443,446)\end{array}$ \\
\hline Kindlin1 & $\begin{array}{l}\beta 1, \beta 3, \\
\beta 6\end{array}$ & $\beta 1:$ TTVVNPKY & $\mathrm{AC}, \mathrm{PD}$ & & $\begin{array}{l}(182, \\
231)\end{array}$ & $\begin{array}{l}\text { co-operates } \\
\text { with talin to } \\
\text { activate } \\
\text { integrin (444) }\end{array}$ \\
\hline Kindlin2 & $\begin{array}{l}\beta 1, \beta 3, \\
\beta 5, \beta 2\end{array}$ & $\begin{array}{l}\beta 1: \text { VTTVVNPKYEG } \\
\beta 3: \text { TSTFTNITYRG }\end{array}$ & $\begin{array}{l}\text { XRAY, } \\
\text { IP }\end{array}$ & $\begin{array}{l}5 \times Q \\
0 \\
5 X Q \\
1\end{array}$ & $\begin{array}{l}(196, \\
253)\end{array}$ & $\begin{array}{l}\text { co-operates } \\
\text { with talin to } \\
\text { activate } \\
\text { integrin (437) }\end{array}$ \\
\hline Kindlin3 & $\begin{array}{l}\beta 1, \beta 2 \\
\beta 3\end{array}$ & $\beta 1:$ TTVVNPKY, $\beta 2$ : membrane-distal NPKF & PD & & $\begin{array}{l}(294, \\
295)\end{array}$ & $\begin{array}{l}\text { co-operates } \\
\text { with talin to } \\
\text { activate } \\
\text { integrin }(248)\end{array}$ \\
\hline Lyn & $\begin{array}{l}\beta 1, \beta 2, \\
\beta 3\end{array}$ & & IP, PD & & $(18,19)$ & Tyr kinase \\
\hline MAPK1 & $\beta 6, \beta 3$ & $\beta 6:$ RSKAKWQTGTNPLYR, $\beta 3$ : EATSTFTN & $\begin{array}{l}\text { IP, } \\
\text { ELISA, } \\
\text { PD }\end{array}$ & & $(6,367)$ & $\begin{array}{l}\text { ERK2, } \\
\text { Ser/Thr } \\
\text { kinase }\end{array}$ \\
\hline MAPK3 & $\alpha \mathrm{V}, \beta 3$ & $\beta 3:$ EATSTFTN & IP, PD & & $\begin{array}{l}(260 \\
367)\end{array}$ & $\begin{array}{l}\text { ERK1, } \\
\text { Ser/Thr } \\
\text { kinase }\end{array}$ \\
\hline MDGI & $\begin{array}{l}\alpha 1, \alpha 2 \\
\alpha 10, \alpha 11\end{array}$ & $\begin{array}{l}\alpha 1: \text { WKIGFFKRPLKKKMEK } \\
\alpha 2: \text { WKLGFFKRKYEKMTKNPDEIDETTELSS } \\
\alpha 10: \text { WKLGFFAHKKIPEEEKREEKLEQ } \\
\alpha 11: \text { WKLGFFRSARRRREPGLDPTPKVLE }\end{array}$ & $\mathrm{Y} 2 \mathrm{H}, \mathrm{B}$ & & $(308)$ & $\begin{array}{l}\text { inhibits } \\
\text { integrin } \\
\text { activity (308) }\end{array}$ \\
\hline Melusin & $\beta 1$ & KLLMIIHDRREFAKFEKEKMNAKWDT & $\begin{array}{l}\mathrm{Y} 2 \mathrm{H}, \\
\mathrm{PD}\end{array}$ & & $(57)$ & \\
\hline Moesin & $\beta 1$ & & PD & & $(449)$ & $\begin{array}{l}\text { competes } \\
\text { with talin } \\
(449)\end{array}$ \\
\hline MYO7A & $\beta 5$ & & IP, PD & & $(268)$ & $\begin{array}{l}\text { promotes cell } \\
\text { migration } \\
(268)\end{array}$ \\
\hline MYO10 & $\begin{array}{l}\beta 1, \beta 3, \\
\beta 5\end{array}$ & $\beta 1:$ WDTGENPIY & $\begin{array}{l}\text { Y2H, } \\
\text { IP, PD }\end{array}$ & & $(496)$ & $\begin{array}{l}\text { integrin } \\
\text { relocalization } \\
\text { to filopodia, } \\
\text { actin binding } \\
\text { (496) }\end{array}$ \\
\hline $\begin{array}{l}\text { Neuropilin } \\
-1\end{array}$ & $\alpha 5$ & & IP & & $(445)$ & \\
\hline Nischarin & $\alpha 5$ & IYILYKLGFFKRSL & Y2H, B & & (9) & $\begin{array}{l}\text { inhibits PAK } \\
\text { kinase } \\
\text { activity (10) }\end{array}$ \\
\hline
\end{tabular}




\begin{tabular}{|c|c|c|c|c|c|c|}
\hline NRK2 & $\begin{array}{l}\beta 1 \mathrm{~A}, \\
\beta 1 \mathrm{D} \\
(\alpha 7 \beta 1 \\
\text { but not } \\
\alpha 5 \beta 1)\end{array}$ & LIWKLLMIIHDRREFAKFEKEKMNAKW & $\begin{array}{l}\text { Y2H, } \\
\text { PD, IP }\end{array}$ & & $\begin{array}{l}(255, \\
256)\end{array}$ & $\begin{array}{l}\text { nicotinamide } \\
\text { riboside } \\
\text { kinase } 2\end{array}$ \\
\hline Numb & $\beta 3, \beta 5$ & membrane-proximal NPxY & PD & & $(65)$ & $\begin{array}{l}\text { clathrin- } \\
\text { mediated } \\
\text { endocytosis } \\
\text { of integrin } \\
(311)\end{array}$ \\
\hline $\begin{array}{l}\text { p120RasG } \\
\text { AP }\end{array}$ & $\alpha 1, \alpha 2$ & $\alpha 2:$ WKLGFFKRKYEKM & $\begin{array}{l}\text { IP, PD, } \\
\mathrm{B}, \mathrm{O}\end{array}$ & & $(275)$ & $\begin{array}{l}\text { recycling of } \\
\text { endocytosed } \\
\text { integrins } \\
(275)\end{array}$ \\
\hline PAK4 & $\beta 5$ & SERSRARYE & $\begin{array}{l}\mathrm{Y} 2 \mathrm{H} \\
\mathrm{PD}\end{array}$ & & $(497)$ & $\begin{array}{l}\text { Ser/Thr } \\
\text { kinase }\end{array}$ \\
\hline Paxillin & $\begin{array}{l}\beta 1, \beta 3, \\
\alpha 4\end{array}$ & $\alpha 4:$ ENRRDSWSY, $\beta 3:$ LYKEATSTFTNITYRGT & $\begin{array}{l}\mathrm{AC}, \mathrm{IP} \\
\mathrm{O}\end{array}$ & & $\begin{array}{l}(180, \\
266 \\
333)\end{array}$ & $\begin{array}{l}\text { adaptor } \\
\text { protein }\end{array}$ \\
\hline PDK1 & $\beta 3$ & phosphorylates T753 & EA & & $(227)$ & $\begin{array}{l}\text { Ser/Thr } \\
\text { kinase }\end{array}$ \\
\hline PKC & $\beta 2$ & $\begin{array}{l}\mathrm{PKC} \delta \text {, PKC } \beta \mathrm{I} / \mathrm{II}, \mathrm{PKC} \alpha \text { and } \mathrm{PKC} \eta \text { phosphorylate } \beta 2 \text { : } \\
\text { S745 and } \beta 2 \text { : T758 } \\
\text { PKC } \alpha \text { phosphorylates T760 }\end{array}$ & EA & & $(128)$ & $\begin{array}{l}\text { Ser/Thr } \\
\text { kinase }\end{array}$ \\
\hline PKD1 & $\beta 3$ & EATSTFTNITYRGT & IP, PD & & $(467)$ & $\begin{array}{l}\text { Ser/Thr } \\
\text { kinase }\end{array}$ \\
\hline Plastin2 & $\beta 1, \beta 2$ & $\begin{array}{l}\text { ß1: DRREFAKFEKEKMNAKWDTG } \\
\beta 2 \text { : LSDLREYRRFEKEKLKSQWN }\end{array}$ & $\begin{array}{l}\text { IP, PD, } \\
\mathrm{O}\end{array}$ & & $(162)$ & $\begin{array}{l}\text { preferentially } \\
\text { binds clasped } \\
\alpha \mathrm{M} \beta 2 \text { and } \\
\text { stabilizes its } \\
\text { inactive state } \\
(441)\end{array}$ \\
\hline Plectin & $\beta 4$ & $\begin{array}{l}\text { FNIII } 1 \text { and 2, N-terminal part of connector between } \\
\text { FNIII } 2 \text { and } 3\end{array}$ & XRAY & $3 \mathrm{~F} 7 \mathrm{P}$ & $(332)$ & $\begin{array}{l}\text { hemidesmoso } \\
\text { me assembly } \\
(234)\end{array}$ \\
\hline PP1C & $\alpha \mathrm{IIb}$ & KVGF & $\mathrm{IP}, \mathrm{PD}$ & & $\begin{array}{l}(13, \\
447)\end{array}$ & $\begin{array}{l}\text { Ser/Thr } \\
\text { phosphatase }\end{array}$ \\
\hline PP2A & $\begin{array}{l}\beta 1 \mathrm{~A} \\
\beta 1 \mathrm{D} \\
\alpha \mathrm{Ilb}\end{array}$ & $\begin{array}{l}\text { dephosphorylates T788 and T789 in } \beta 1 \mathrm{~A} \\
\alpha \mathrm{IIb} \text { : KVGFFKR }\end{array}$ & $\begin{array}{l}\text { IP, PD, } \\
\text { EA }\end{array}$ & & $\begin{array}{l}(176 \\
225)\end{array}$ & $\begin{array}{l}\text { Ser/Thr } \\
\text { phosphatase }\end{array}$ \\
\hline PTPN1 & $\beta 3$ & & IP & & (17) & $\begin{array}{l}\text { Tyr } \\
\text { phosphatase }\end{array}$ \\
\hline Rab21 & $\begin{array}{l}\alpha 1, \alpha 2 \\
\alpha 5, \alpha 6 \\
\alpha 11, \alpha 16\end{array}$ & GFFKR & $\begin{array}{l}\mathrm{Y} 2 \mathrm{H} \\
\mathrm{IP}, \mathrm{O}\end{array}$ & & $\begin{array}{l}(275, \\
331)\end{array}$ & small GTPase \\
\hline Rab25 & $\beta 1$ & & IP, PD & & $(72)$ & small GTPase \\
\hline Rab34 & $\beta 3$ & & PD & & $(418)$ & small GTPase \\
\hline RACK1 & $\begin{array}{l}\beta 1, \beta 2, \\
\beta 5, \alpha 4 \\
\alpha \mathrm{V}\end{array}$ & $\beta 2:$ KALIHLSDLREYRRFEKEKL & Y2H, IP & & $\begin{array}{l}(258, \\
498)\end{array}$ & $\begin{array}{l}\text { adaptor } \\
\text { protein }\end{array}$ \\
\hline Radixin & $\beta 2$ & CWKALIHLSDLREYRRF & $\begin{array}{l}\text { PD, } \\
\text { ELISA }\end{array}$ & & $(433)$ & \\
\hline RanBP9 & $\beta 2, \beta 1$ & & $\begin{array}{l}\mathrm{Y} 2 \mathrm{H} \\
\mathrm{PD}\end{array}$ & & $(105)$ & $\begin{array}{l}\text { Ran GTPase } \\
\text { binding }\end{array}$ \\
\hline RNF181 & $\alpha \mathrm{IIb}$ & KVGFFKR & PD & & $(60)$ & $\begin{array}{l}\text { ubiquitin } \\
\text { ligase }\end{array}$ \\
\hline
\end{tabular}




\begin{tabular}{|c|c|c|c|c|c|c|}
\hline SHARPIN & $\begin{array}{l}\alpha 1, \alpha 2, \\
\alpha 5, \alpha \mathrm{L} \\
\alpha \mathrm{M}, \alpha \mathrm{D}\end{array}$ & GFFKR & PD, IP & & $\begin{array}{l}(143, \\
343, \\
359)\end{array}$ & $\begin{array}{l}\text { inhibitor of } \\
\text { integrin } \\
\text { activation } \\
(359)\end{array}$ \\
\hline SHC1 & $\begin{array}{l}\alpha \mathrm{V} \beta 3 \\
\beta 1, \beta 4\end{array}$ & $\begin{array}{l}\text { DTANNPL[pY]KEATSTFTNIT[pY]RGT; } \beta 4: \text { Y1440 } \\
\text { binds to She SH2, Y1526 binds to She PTB }\end{array}$ & $\begin{array}{l}\text { IP, } \\
\text { NMR, } \\
\text { O }\end{array}$ & 2L1C & $\begin{array}{l}(99, \\
108, \\
227, \\
243)\end{array}$ & $\begin{array}{l}\text { adaptor } \\
\text { outside-in } \\
\text { signaling (93) }\end{array}$ \\
\hline Shp2 & $\beta 4$ & Y1494 & IP & & $\begin{array}{l}(43, \\
478)\end{array}$ & $\begin{array}{l}\text { Tyr } \\
\text { phosphatase }\end{array}$ \\
\hline Skelemin & $\alpha \mathrm{Illb}, \beta 3$ & $\begin{array}{l}\alpha I I b: \text { VGFFKRNRPP } \\
\beta 3: \text { KLLITIHDR }\end{array}$ & $\begin{array}{l}\text { Y2H, } \\
\text { PD, } \\
\text { NMR, } \\
\text { B, O }\end{array}$ & & $\begin{array}{l}(109, \\
337, \\
360)\end{array}$ & \\
\hline SNX17 & $\begin{array}{l}\beta 1, \beta 3, \\
\beta 5, \beta 6, \\
\beta 2\end{array}$ & membrane-distal NxxY & PD, IP & & $\begin{array}{l}(50, \\
314, \\
411, \\
442)\end{array}$ & $\begin{array}{l}\text { integrin } \\
\text { recycling } \\
\text { from early } \\
\text { endosomes } \\
(411)\end{array}$ \\
\hline SNX31 & $\begin{array}{l}\beta 5, \beta 6, \\
\beta 1, \beta 2, \\
\beta 3\end{array}$ & membrane-distal NxxY & PD, IP & & $(442)$ & $\begin{array}{l}\text { integrin } \\
\text { recycling } \\
\text { from early } \\
\text { endosomes } \\
(442)\end{array}$ \\
\hline SRC & $\beta 3$ & C-terminal RGT & $\begin{array}{l}\text { XRAY, } \\
\text { IP, AC, } \\
\text { PD }\end{array}$ & $4 \mathrm{HXJ}$ & $\begin{array}{l}(18, \\
469)\end{array}$ & Tyr kinase \\
\hline SYK & $\beta 2$ & & IP & & $\begin{array}{l}(448, \\
476)\end{array}$ & Tyr kinase \\
\hline $\begin{array}{l}\text { Syntrophin } \\
1\end{array}$ & $\beta 5$ & KFNKSYNGTVD & $\mathrm{O}$ & & $(47)$ & \\
\hline \multirow{2}{*}{ Talin1 } & $\begin{array}{l}\text { IBS1: } \\
\beta 1 \mathrm{~A}, \\
\beta 1 \mathrm{D}, \beta 2, \\
\beta 3, \beta 5 \\
\beta 6, \beta 7\end{array}$ & $\begin{array}{l}\text { IBS1: } \\
\beta 1 \text { A: NAKWDTGENPIYKS } \\
\beta 1 D: \text { NAKWDTQENPIYKS } \\
\beta 3: \text { TIHDRKEFAKFEEERARAKWDTANNPLYKEA }\end{array}$ & $\begin{array}{l}\text { IBS1: } \\
\text { XRAY, } \\
\text { NMR, } \\
\text { PD, B }\end{array}$ & \multirow{2}{*}{$\begin{array}{l}\text { IBS1: } \\
\text { 1MK } \\
7\end{array}$} & $\begin{array}{l}\text { IBS } 1: \\
(15,65 \\
150 \\
237 \\
442)\end{array}$ & \multirow[t]{2}{*}{$\begin{array}{l}\text { integrin } \\
\text { activation } \\
(66), \text { adaptor } \\
\text { protein, actin } \\
\text { binding }\end{array}$} \\
\hline & $\begin{array}{l}\text { IBS2: } \\
\beta 1, \beta 2 \\
\beta 3, \beta 7\end{array}$ & $\begin{array}{l}\text { IBS2: } \\
\beta 1: \text { KLLMIIHDRREFAKFEKEKMNAK } \\
\beta 2: \text { KALTHLTDLREYRRFEKEKLKSQ } \\
\beta 3: \text { KLLITIHDRKEFAKFEEERARAK } \\
\beta 7: \text { RLSVEIYDRREYRRFEKEQQQLN }\end{array}$ & $\begin{array}{l}\text { IBS2: } \\
\text { PD, } \\
\text { ELISA, } \\
\text { B, O }\end{array}$ & & $\begin{array}{l}\text { IBS2: } \\
(159 \\
290 \\
370)\end{array}$ & \\
\hline Talin2 & $\begin{array}{l}\text { IBS1: } \\
\beta 1 \mathrm{~A} \\
\beta 1 \mathrm{D}, \beta 3\end{array}$ & $\begin{array}{l}\beta 1 \mathrm{~A}: \text { NAKWDTGENPIYKS } \\
\beta 1 D: \text { NAKWDTQENPIYKS } \\
\beta 3: \text { TIHDRKEFAKFEEERARAKWDTANNPLYKEA }\end{array}$ & $\begin{array}{l}\text { XRAY, } \\
\text { NMR, } \\
\text { ITC }\end{array}$ & $\begin{array}{l}3 \mathrm{G} 9 \\
\mathrm{~W}\end{array}$ & $(15,16)$ & $\begin{array}{l}\text { adaptor } \\
\text { protein, actin } \\
\text { binding }\end{array}$ \\
\hline Tensin-1 & $\beta 1 \mathrm{~A}, \beta 3$ & $\begin{array}{l}\beta 1 \mathrm{~A}: \text { KWDTGENPIYKS } \\
\beta 3: \text { KWDTANNPLYKE }\end{array}$ & B & & $(284)$ & actin binding \\
\hline Tensin-2 & $\begin{array}{l}\beta 3, \beta 5 \\
\beta 7, \beta 1 \mathrm{~A}\end{array}$ & & PD & & $(65)$ & actin binding \\
\hline Yes & $\begin{array}{l}\beta 3 \beta 1 . \\
\beta 2\end{array}$ & & IP, PD & & $\begin{array}{l}(18,19 \\
314)\end{array}$ & Tyr kinase \\
\hline$\alpha$-actinin & $\begin{array}{l}\beta 1, \beta 2, \\
\beta 3\end{array}$ & $\beta 2:$ RRFEKEKLKSQ & $\begin{array}{l}\mathrm{AC}, \mathrm{IP} \\
\mathrm{O}\end{array}$ & & $\begin{array}{l}(318, \\
381)\end{array}$ & $\begin{array}{l}\text { actin binding } \\
(318)\end{array}$ \\
\hline
\end{tabular}




\section{Chapter 5. Proliferation and YAP/TAZ signaling}

\section{Proliferation}

Integrins are usually considered to be cell-matrix receptors. This might be mistaken as a passive "gluing" to a substrate, offering a mere structural link to the cytoskeleton. Telling the story of the paxillin discovery, Christopher Turner described this as the "dogma of the time" for integrin-mediated adhesions (103). Fittingly, integrins possess no kinase domain, potentially enhancing this belief at the beginning of integrin adhesion research. Still today, integrins and integrin-mediated adhesions are sometimes just regarded as a structural link to actin und intermediate filaments. Nevertheless, there is no doubt about the signaling capacity of integrincontaining adhesive structures. Already early on, integrin-mediated adhesions were shown to have elevated levels of tyrosine phosphorylation in v-Src transformed cells (161). On the other hand, endothelial (286) and epithelial cells (147) undergo apoptosis after detachment from the ECM (i.e. anoikis). Both examples, anoikis and increased phosphorylation after v-Src mediated immortalization, highlight the link of integrin signaling to proliferation. Anoikis is regulated by a FAK-p53 signaling axis (259), while YAP/TAZ proteins regulate substrate stiffnessdependent proliferation (see below). Especially the YAP/TAZ pathway has attracted a lot of attention in the last years, as it forms a link between mechanical input and cell proliferation. However, it is interesting to note that the MRTF/SRF signaling pathway acts in parallel to and shares certain target genes with the YAP pathway (140). Additionally, the MRTF/SRF pathway targets also genes independently of YAP, which mediate a phenotype often associated with increased YAP/TAZ signaling $(140,188)$.

\section{YAP/TAZ}

As integrins are increasingly recognized as mechanosensors, we want to discuss the proliferative signaling of the integrin-dependent mechanotransducers YAP/TAZ in more detail. For many years, the co-transcriptional regulators YAP and TAZ were mainly associated with the Hippo signaling cascade controlling organ size in vivo and contact inhibition of proliferation in cell culture (322). More recently, YAP/TAZ also emerged as important mechanotransducers, sensing a variety of mechanical inputs and integrating them into output signals controlling proliferation and stem cell differentiation (116). Stiff substrates in 2D and 3D, large adhesive areas and increased blood pressure are examples of physical parameters that induce YAP/TAZ activity by increasing their nuclear localization (323). At the same time, active YAP/TAZ increases the expression not only of proteins driving proliferation, but also of focal adhesion and actin organization (305), establishing a positive feedback ensuring persistent YAP/TAZ 
activation. Mechanistically, YAP/TAZ proteins were found to be inactive as long as they reside in the cytoplasm. This is the case, when they are Ser/Thr phosphorylated, leading to the formation of a complex with phosphoSer-binding 14-3-3 adapters (116). YAP/TAZ proteins are phosphorylated by the large tumor suppressor gene 1 and 2 (LATS1/2) as part of the canonical Hippo signaling pathway. Dephosphorylated YAP/TAZ instead is enriched in the nucleus, where it binds transcription factors like TEAD1 (305). This YAP/TAZ activation is supported by a $\beta 1$ integrin-Src axis and potentially explains the proliferative effects of $\beta 1$ integrin expression (376). However, the precise mechanism of YAP/TAZ activation remains controversial, as some groups found also integrin-independent cell adhesion on poly-L-lysinecoated substrates to cause YAP/TAZ activation $(100,499)$. These reports argued that the actin network integrity is necessary for nuclear localization of YAP/TAZ. Recent publications might now be able to reconcile these different findings: Elosegui-Artola and colleagues (121) found that mechanical stretching of the nuclear membrane opens nuclear pore complexes (NPCs). An increased diameter of NPCs then allows an easier entry of YAP proteins into the nucleus. This size-dependent mechanism of YAP transport was supported by experiments showing that increasing the size of the YAP protein by attachment of one or two GFP reduces its nuclear localization. The importance of mechanical force on the nucleus for YAP/TAZ activation was also shown by Shiu, Aires and colleagues (399). Their work indicated that an actin cap, spanning over the nucleus and thereby flattening it, applied mechanical force on the nucleus, leading to YAP activation. This actin cap relied on $\beta 1$ integrin localization in the perinuclear region. Thus, it is evident that both $\beta 1$ integrin signaling as well as actin integrity are needed for force application on the nucleus, which then facilitates the nuclear localization of YAP/TAZ. Apart from this mechanism, also other signaling cascades for YAP/TAZ signaling have been introduced recently. Meng and colleagues (285) showed that focal adhesions on soft substrates activate RAP2, leading to a deactivation of RhoA and activation of LATS1/2. As a consequence, YAP becomes phosphorylated and remains in the cytoplasm. Thus, separate pathways might work together to balance activation and inactivation of YAP/TAZ. However, as mentioned in the beginning, it is important to better analyze these pathways in order to understand the potential crosstalk with the MRTF signaling cascade. Interestingly, both YAP/TAZ and MRTF cascades involve integrin-mediated structural linkage and signaling, rendering these pathways fascinating examples of cellular signaling, where mechanical and biochemical inputs are sensed and integrated by integrins and their downstream targets. 


\section{Chapter 6: The concept of mechanosensing: linking integrin-dependent cell adhesions to}

signaling

Cells are in an active crosstalk with their surrounding environment. Cells integrated in a functional tissue receive signals not only from their neighboring cells, but also sense the global forces and metabolic state of a tissue. Although chemical signaling has highly important roles in cellular homeostasis, also physical signals including mechanical cues are essential for proper tissue functions. How do cells sense mechanical signals?

Integrin-mediated adhesions have a central role in cellular mechanosensing: they are physical links between individual cells and their surrounding extracellular matrix (419). Cell-matrix adhesions can thus be considered mechanical connectors. On the intracellular side, they are linked to the actomyosin machinery via the cell cytoskeleton, and on the extracellular side they are coupled to extracellular scaffolds formed by proteins such as fibronectin and collagen, containing specific attachment sites for integrin receptors (177, 458, 493). Cell-matrix adhesions are considered mechanosensitive, as their size, composition and signaling capacity are known to be affected by mechanical load and substrate stiffness $(123,366)$.

Integrins are among the most studied mechanosensory receptors. In cellular mechanosensing, a mechanical signal is received by a mechanoreceptor, which is capable of translating the signal into a chemical cue (82). The chemical signal may then affect cellular processes such as gene expression. This process is called mechanotransduction (79).

What do we know about the details of integrin-mediated mechanosensing? First of all, integrins have several different conformations, as discussed in Chapter 2. The regulation of integrin conformation is the first level of mechanosensing in cell adhesion (Figure 8). Although integrin conformation can be modulated by chemical factors, the full activation of integrins requires mechanical signals (208). However, as indicated in Chapter 2, it is still under investigation whether mechanisms of integrin activation found in well-studied integrins can be easily transferred to others. Therefore, we should not expect the same scheme of conformational regulation to be applicable for all integrin family members.

The second level in integrin-mediated mechanosensing is the integrin-ligand binding (Figure 8). While protein-protein interactions typically have a decreased lifetime under mechanical 
load, the bond between fibronectin and integrins $\alpha 5 \beta 1$ and $\alpha \mathrm{V} \beta 3$ has been found to function as a catch-bond, becoming stronger when force is applied $(146,233,291)$.

The third level of integrin-mediated mechanosensing relates to the intracellular adapter proteins (Figure 3, Table 1). The cytoplasmic domain of integrin acts as a ligand for several adapter proteins, including talin $(177,228)$, kindlin (371), sharpin (359), tensin (156) and a-actinin (329). These adapter proteins link integrins to the cytoskeleton, but also mediate cellular signals. Importantly, the adapter proteins also act as mechanosensory elements (for review, see $(153,194,208))$. The best studied example is talin, which connects to integrin via its N-terminal head domain and to F-actin via the C-terminal rod domain $(169,228)$. This exposes talin to mechanical load, facilitating conformational changes. As a result, cryptic binding sites for other adhesion proteins become exposed and the particular adhesion site is reinforced (160). Proposed force-regulated talin binding partners include vinculin $(135,160,206,247,353,482)$, DLC1 $(178,491)$, RIAM $(170,244,477)$, and paxillin (384), but considering the size and complexity of talin, it is likely that additional force-regulated talin interactions exist $(169,301)$. As mentioned above, the situation for paxillin is unique, as it is recruited to the talin-rod by short helical elements positioned in its flexible $\mathrm{N}$-terminal domain, that show affinity towards FAK and parvin as well as the GIT/PIX/PAK regulatory complex. On the other hand, tensionmediated paxillin recruitment to adhesions (384) is regulated at the level of the proximal talinbinding NPXY motif of the integrin (335), as well as the integrin-tail recruited kindlin (149, 437). While the mechano-dependent recruitment of paxillin to integrin-adhesions is still not fully understood, it illustrates well how the connectivity of different intracellular adapter proteins allows different signaling outputs in responses to mechanical perturbations.

Although these signaling outputs appear to create an on/off signal, it is also important that this mechanosensing system can be used in different cellular contexts, exhibiting largely different force regimes. Our recent study focusing on the talin rod revealed the tailoring of talin properties to have significant effects on cellular mechanosensing (353). We found mechanically weakened talin to decrease cellular traction force. Even more interestingly, we noticed the recognition of extracellular matrix proteins to be altered in cells expressing mechanically weakened talin compared to cells expressing the wild-type protein. Therefore, it appears that mechanical signals are instrumental in controlling the environmental sensing via integrins. 
The mechanically induced conformational changes in intracellular proteins contributing to cellmatrix adhesion have been discussed in our previous review (208). We are only at the beginning to understand the mechanoregulation of adhesion signaling at the molecular level. However, the mechanisms are taking shape: i) opening of binding sites due to mechanical load (example: vinculin binding sites in talin rod $(115,160,206)$, ii) disappearance of binding sites due to mechanical load (DLC1 - talin, $(178,252)$ ), iii) phosphorylation of mechano-exposed regions within proteins (p130Cas) (385), iv) and proteolytic cleavage of partially unfolded proteins (4). Figure 8 aims to summarize these mechanisms. These regulatory events, combined with the high number of components involved, makes the creation of a comprehensive model of adhesion signaling challenging. In addition, new mechanisms still emerge as e.g. the competition between kank2 and actin for talin binding, leading to a modulated force transmission to integrins (420). The motor-clutch model emerged over the years as a promising framework to explore integrin dependent mechanotransduction $(73,123)$. In this model, molecular clutches link F-actin to the substrate and mechanically resist myosin-driven F-actin retrograde flow (73). This model might in future incorporate additional features to model real integrin-mediated adhesions more closely.

An increasing number of reports indicate that mechanotransduction on substrates of increasing stiffness follows a sigmoidal mechanosensitivity, creating a behavioral switch at a substrate stiffness around $5 \mathrm{kPa}$. Adhesion maturation, nuclear translocation of YAP or cell spreading are all suppressed on substrates below $5 \mathrm{kPa}$ and increase from there within a narrow stiffness range to reach plateau levels. Roca-Cusachs and coworkers successfully linked this behavior to the motor-clutch model (122). Importantly, this on/off mechanoswitch also implies that studies on glass substrates, with a stiffness in the MPa range, might miss important changes in mechanotransduction occurring around the physiologically relevant $5 \mathrm{kPa}$ stiffness range. According to the motor-clutch model, integrin-ligand affinity is a parameter that might shift the onset of mechanotransduction from $5 \mathrm{kPa}$ to softer or stiffer substrates. This also implies that seemingly redundant integrin-ligand interactions on glass substrates might cause specific, differential effects at physiological stiffness values. We recently showed $\alpha \mathrm{V} \beta 3$ integrin to have a higher affinity for vitronectin than for fibronectin, leading to differential mechanotransduction on the respective ligands (31). We envision that the increased interest in the mechanical regulation of integrins will lead to the discovery of further force- and stiffness-dependent integrin-ligand interactions. At the same time, it is clear that also integrin adapters are an intrinsic part of the mechanosensory machinery. Talin has several cryptic binding sites, that 
open under mechanical stretch, and is therefore not only of interest as integrin activator, but also as mechanosensor and mechanotransducer (see above) (170, 353, 354). Interestingly, the mechanotransduction by talin is isoform-specific, with talin 2 increasing the ability of cells to spread on 1-2 $\mathrm{kPa}$ substrates compared to talin1 (26). This difference can be attributed to the subdomains R1-R3 in the talin rod domain (indicated as yellow rectangles in Figure 6). Interestingly, several studies found these subdomains to be relevant for mechanosensing and force-dependent structural rearrangements. While talin is involved in integrin activation, it was demonstrated that also an integrin inhibitor, Thy-1, modulates mechanosensing (see Figure 3E) (136). Therefore, mechanosensing by integrins appears to rely on the proper balance between ligand binding and unbinding.

\section{Chapter 7: Role of integrins in viral and bacterial infections}

Integrins are best known as receptors contributing to cellular attachment. However, they also act as receptors for viruses and bacteria and are otherwise involved in pathogenic processes.

There are numerous known viruses exploiting integrins for their attachment to the cell, the virus entry into the cell as well as endosome escape (reviewed in (201)). Many viruses display an RGD sequence on their surface, which enables integrin binding. Adenoviruses utilize integrin $\alpha \mathrm{V}$ for virus internalization (464). Binding of the adenovirus to integrin appears to induce a conformational change of integrin into an extended conformation; simultaneously a conformational rearrangement is observed also in the virus capsid (261). Similarly, integrin $\alpha \mathrm{V} \beta 3$ has been identified as a cellular receptor mediating both the cell adhesion and entry of Kaposi's sarcoma-associated Herpes virus into target cells (152). Another interesting group of viruses utilizing RGD-dependent integrins are enteroviruses, being among the most common human pathogens. Within the group of enteroviruses, only a handful of virus strains appear to utilize integrins in cell recognition. One of them is the coxsackie virus A9 (CVA9), which shows preferential binding to $\alpha v \beta 6$ with a low nanomolar $K_{d}$ (393). However, in this case integrin activation might actually not promote virus internalization (393). Among other RGDpossessing viruses, HIV utilizes $\alpha 4 \beta 7$ via the RGD tripeptide in the V2 loop of gp120 to infect the cell (23), and Ebola virus appears to bind $\alpha 5 \beta 1$-integrin for cell entry.

Viruses do not always depend on RGD to utilize integrins in their propagation cycle. Rotavirus infection was blocked with peptides containing the $\alpha 4$ integrin ligand sequences Tyr-Gly-Leu and Ile-Asp-Ala. These peptides eliminated virus binding to $\alpha 4$ integrins and infectivity (171). 
Another non-RGD integrin-dependent virus is Ross River virus, which appears to utilize the collagen-binding integrin $\alpha 1 \beta 1$ (263). Some integrin-interacting enteroviruses do not contain RGD-like peptides, such as echovirus 1 (41). Interestingly, this virus appears to prefer binding to closed $\alpha 2 \beta 1$ integrin, and the inactivating integrin mutation E336A further enhanced this integrin binding (213). Echovirus 1 makes a large contact with the I-domain, with MIDAS site not being involved in binding (226). This virus does not depend on the integrin $\alpha$-subunit during the later events of virus entry - the virus can infect cells even if the $\alpha 2$-tail is swapped or deleted (40). Notably, the binding of echovirus 1 appears to induce cellular signaling via focal adhesion kinase (379).

The natural tendency of integrins to cluster as a response to extracellular signals is complementary to the repetitive structure found in many virus capsids. For example, enteroviruses are $\sim 30 \mathrm{~nm}$ in diameter, and contain 60 copies of VP1-VP4 capsid proteins. Therefore, the RGD sequences are displayed on the virus capsid almost perfectly in line with the density of clustered integrins observed in living cells: Changede et al. (75) reported $\sim 100 \mathrm{~nm}$ clusters containing $\sim 50$ activated $\beta 3$-integrins in the early adhesions under a wide variety of conditions on RGD surfaces. Thus, the regular and dense arrangement of integrin ligands on the virus particle offers a fascinating platform for the active integrin-mediated communication between cells and viruses.

The studies of Echovirus 1 have revealed, that virus binding can lead to integrin clustering without activation (213). Further, clustering of nonactivated integrins induces transient phosphorylation of FAK and paxillin in a PKC $\alpha$-dependent, but talin-independent manner (379). These findings suggest that virus-induced clustering of integrins can activate FAK without conformational integrin activation.

Coxsackievirus B3 is the most viral cause of myocarditis (for review, see (130)). Activation of Akt during coxsackievirus B3 infection has been shown to take place through a PI3K-dependent pathway (127). Inhibition of integrin-linked kinase (ILK) activity and expression significantly blocked coxsackievirus B3-triggered Akt phosphorylation on Ser473 without effect on Thr308 phosphorylation. As a consequence, ILK inhibition lead to a significant decline in coxsackievirus B3 RNA transcription, viral protein synthesis, and virus progeny release. 
Integrins are involved also in bacterial infections, and the following examples provide some insights into the mechanisms. A more extensive summary of bacterial species engaging integrins within the infection cycle can be found in a review by Hauck et al. (186).

Shigella bacteria cause shigellosis, a common intestinal infection leading to diarrhea and fever. Using $\mathrm{CHO}$ cells expressing integrin subunits, Watarai et al. showed that integrin $\alpha 5 \beta 1$ promotes the entry of the Shigella flexneri bacteria (455). They demonstrated IpaB, IpaC and IpaD proteins to bind to $\alpha 5 \beta 1$. Interestingly, Shigella appears to utilize also other components of the cell adhesion complex during its invasion, including vinculin (326) and ILK (224).

Staphylococcus aureus is a common cause for respiratory infections. Most clinical isolates of S. aureus express the fibronectin-binding proteins FnBP-A and FnBP-A (330). Binding of fibronectin by FnBPs is essential for the bacterial invasion, with fibronectin functioning as a bridging molecule linking FnBP to integrin $\alpha_{5} \beta_{1}$ (reviewed in (186)). This leads to the active intake of the bacteria, which does not require other bacterial factors, since even FnBP-coated polystyrene beads are internalized by cells (405).

Invasins are a class of bacterial proteins associated with the penetration of bacteria into cells. Isberg et al. showed that $\alpha 3 \beta 1, \alpha 4 \beta 1, \alpha 5 \beta 1$, and $\alpha 6 \beta 1$ all bind the Yersinia pseudotuberculosis invasin protein (210). The integrin-binding domain was mapped to a 192-aa C-terminal region of invasin that does not contain any RGD sequence (251). This integrin-binding domain was found sufficient to allow bacterial entry into mammalian cells (358). Later, beads coated with a larger invasin derivative comprising the C-terminal 497 amino acids were found to be internalized more efficiently than those bearing only 197 C-terminal residues. This seemed to be explained by the homomultimerization of the immobilized invasin fragment (106), suggesting a role of integrin clustering also in bacterial invasion.

The last example of integrin-bacteria-interaction demonstrates how bacterial cells can modulate tissue structure to support bacterial colonization. In some human tissues, the turnover rate of cells can be high, and e.g. the intestinal epithelium self-renewal is completed within 2-3 days (342). Slowing down this epithelial turnover can support bacterial colonization during an infection (212). Shigella flexneri can reinforce host cell adherence to the basal membrane via ILK (224). The interaction between ILK and the effector protein OspE increases cell surface levels of $\beta 1$ integrin and suppresses phosphorylation of focal adhesion kinase and paxillin. As 
a result, the reduced adhesion turnover and suppressed detachment of infected cells enables Shigella to colonize the epithelium. In a similar fashion, some bacteria such as $N$. gonorrhoeae can bind to human carcinoembryonic antigen-related cell adhesion molecules (CEACAMs), promoting enhanced host cell adhesion via integrin $\beta 1$ activation (297).

In summary, integrins are important mediators of viral and bacterial infections, and integrinmediated attachment, internalization as well as control over tissue integrity are central mechanisms in pathogenic processes. One may thus ask if integrins are employed more frequently as receptors for pathogens than other cell surface proteins. This is not straightforward to address: Although integrins are widely utilized by viruses and bacteria $(280,413)$, also numerous other known viral receptors are known (173). Potentially the intense study of integrins has lead to their frequent identification as virus receptors. In any case, it appears remarkable, that viruses, having a highly variable structure and shape, can exploit integrins in cell recognition. One such example is the utilization of integrins by adenoviruses and enteroviruses, two different classes of viruses with different evolutionary origin, showing both five integrin binding sites located in pentagonic assembly with spacing of $\sim 60 \AA$ (reviewed by Stewart \& Nemerow (413)). We therefore speculate that integrin clustering and integrin activation are suitable cellular mechanisms for the exploitation by infectious agents, and that the integrin-pathogen interactions thus offer potential targets for development of novel drugs.

\section{Chapter 8: Integrins and diseases}

Integrins are central for the integrity of the tissues, cellular adhesion and cell-matrix interactions, and it is therefore not surprising that several diseases are associated with defects in integrins. These integrin-related diseases are an active target for drug development, and a search with the term "integrin" revealed 151 studies in ClinicalTrials.gov, reflecting the importance of this research field. A significant portion of these studies ( $30 \%)$ are focusing on integrin-targeting drugs. The first integrin-targeting drug entering the market 1994 was Abciximab (ReoPro), a $47 \mathrm{kDa}$ Fab fragment against $\alpha \mathrm{IIb} \beta 3$ based on the monoclonal antibody developed by Coller et al. 1983 (91). This antibody also binds $\alpha v \beta 3$ (430) and $\alpha \mathrm{M} \beta 2$ (403); it is used to prevent blood clots during the opening of blood vessels in the heart. Eptifibatide (Integrilin) is a cyclic heptapeptide derived from a barbourin protein found in the venom of the southeastern pygmy rattlesnake. This peptide targets $\alpha \operatorname{Ilb} \beta 3$ and is used to reduce the risk of acute cardiac ischemic events (183, 435). It was launched in Europe 1999 and in USA 1998. 
Tirofiban (Aggrastat) is small molecule inhibitor for $\alpha \mathrm{IIb} \beta 3$. It was approved in USA 2000 and in Europe 1999 for the treatment of acute coronary syndrome. Natalizumab (Tysabri) is a humanized monoclonal antibody against $\alpha 4$-integrin used in the treatment of multiple sclerosis (339) and Crohn's disease (167). Natalizumab is thought to prevent immune cells from crossing blood vessel walls to reach the affected organs. The most recent integrin-targeted therapeutic antibody accepted for clinical use is Vedolizumab (Entyvio), which is a humanized monoclonal antibody specifically binding to the $\alpha 4 b 7$ integrin and blocking the interaction of $\alpha 4 b 7$ integrin with mucosal addressin cell adhesion molecule-1 (407). This leads to inhibition of the migration of memory T-lymphocytes across the endothelium into inflamed gastrointestinal tissue (372). Vedolizumab is approved for treating patients with ulcerative colitis or Crohn's disease.

The following paragraphs provide insights into the importance of integrins and integrin activation in various diseases.

\section{Integrins and skin diseases}

Integrins are important for the integrity of the skin. Experiments with genetically manipulated mice have shown that deletion of $\alpha 6$ or $\beta 4$ leads to disappearance of hemidesmosomes and the impairment of the epidermal adhesion to the underlying basement membrane $(114,155,307)$. Even modest mechanical stress causes peeling of the epidermis from its underlying tissue in these animals. In humans the equivalent disease is known as junctional epidermolysis bullosa, and the associated genetic factors are reviewed in (185).

Mice deficient in $\alpha 3$ integrin have only a mild skin phenotype: the epidermis of $\alpha 3$ integrindeficient mice has normal morphology and other characteristics $(111,192,235)$. Also the hemidesmosomes of $\alpha 3$ integrin-deficient mice appear intact, and most regions of the basal membrane are coherent. The only defects observed in these animals are microblisters in the limb skin regions due to ruptures within the basal membrane.

As $\beta 1$ integrin is essential for mouse development $(132,412)$, the role of $\beta 1$ in skin epithelium has been studied using conditional knockout technology $(56,352)$. Mice with keratinocytespecific knockouts for $\beta 1$ integrin exhibit severe skin blistering and hair defects (352). In detail, a massive failure of basement membrane organization was observed, hemidesmosomes were instable and hair follicle keratinocytes failed to remodel the basement membrane. Also, Brakebusch et al. generated mice with a keratinocyte-restricted deletion of the $\beta 1$ integrin using 
the cre-loxP method (56). This resulted in hair loss and hair follicle abnormalities, and the epidermis of the skin became hyperthickened. The loss of $\beta 1$ also caused a reduced $\alpha 6 \beta 4$ expression in basal keratinocytes and a decreased number of hemidesmosomes. Moreover, disruption of the basement membrane and blister formation were observed at the dermalepidermal junction.

Integrins have also been a target for drug development for skin diseases. Efalizumab (Raptiva) is a recombinant humanized monoclonal antibody designed to treat autoimmune diseases (462), that was originally authorized for the treatment of psoriasis by EMEA 2004 and FDA 2003 (168). Efalizumab binds to the integrin $\alpha \mathrm{L}$ subunit of the $\alpha \mathrm{L} \beta 2$ integrin specific for leukocytes (189). However, the marketing of efalizumab was suspended 2009 due to side effects such as Guillain-Barré and Miller-Fisher syndromes, encephalitis, encephalopathy, meningitis, sepsis and opportunistic infections.

While integrin activation by intracellular proteins is discussed elsewhere in this review, in the context of skin diseases, it is relevant to pinpoint the connection between kindlin and skin diseases. The best-known disease associated with kindlins is Kindler syndrome, involving the loss of kindlin-1 expression in epidermis (402). Gene knockout of Fermt1, the gene encoding kindlin-1, in mice caused skin atrophy and lethal intestinal epithelial dysfunction (444).

\section{Cancer}

Integrins have a central role in cancer, as reviewed previously $(107,374)$, and it is not possible to give a comprehensive overview within this article. Instead, we provide here selected examples of the importance of integrin activation in cancer.

Felding-Habermann et al. (134) demonstrated activated integrin av $\beta 3$ mutant D723R to support tumor cell arrest in the circulation through the interaction with platelets. This activated $\alpha v \beta 3$ was found to be expressed by metastatic human breast cancer cells, leading to metastatic lesions. Expression of the constitutively activated integrin mutant $\alpha v \beta 3-D 723 R$ promoted metastasis in a mouse model. These results support a model where breast cancer cells can exhibit a platelet-interactive and metastatic phenotype controlled by the activation of integrin $\alpha v \beta 3$. Beside this mechanism, $\alpha v \beta 3$ integrin might have additional and parallel modes of action supporting cancer progression and metastasis. Several studies indicated that $\alpha v \beta 3$ integrin and 
VEGF receptor signaling act synergistically to promote angiogenesis $(101,274)$, an important part of tumor progression. This link between $\alpha v \beta 3$ integrin and VEGF signalling motivated clinical trials with Cilengitide, a specific inhibitor of $\alpha v \beta 3$ and $\alpha v \beta 5$ integrin. Also glioblastoma express $\alpha v \beta 3$ integrin, in contrast to the healthy surrounding tissue, making $\alpha v \beta 3$ integrin inhibition a promising strategy for the treatment of this cancer. Accordingly, orphan designation for Cilengitide (EU/3/03/184) was granted by the European Commission on 14 January 2004 to Merck KGaA, Germany, for the treatment of glioma. Unfortunately, the drug was later removed from the market, as the phase 3 study showed no positive effect in glioblastoma treatment (415). A mechanism explaining the failure of Cilengitide in this clinical trial is still missing, although it was recognized later on that a low dosing of Cilengitide activates angiogenesis in mice rather than preventing it (362). This surprising effect might be explained by systemic effects, but the partial agonism of high-affinity integrin inhibitors like Cilengitide might still challenge this therapeutic strategy. In fact, we recently showed that the correlation of the different ectodomain conformations of integrins with immobilized ligand binding is still challenging. We revealed that $\alpha v \beta 3$ integrin binds vitronectin in the extended-closed conformation, but fibronectin only in the extended-open conformation (31). Thus, rational drug design could benefit from further research efforts focusing on integrin-ligand interactions that take into account the conformational flexibility of the integrin ectodomain. Furthermore, this research should be performed appreciating the non-redundancy of these interactions.

Chronic myeloproliferative neoplasia comprises several sub-entities, including polycythemia vera (PV), essential thrombocytosis (ET), primary myelofibrosis (PMF) and others (436). Chronic myeloproliferative neoplasia caused by the V617F mutant of Janus kinase 2 (JAK2) commonly displays abnormal integrin expression on platelets, erythrocytes and leukocytes (118). A recent study found JAK2-V617F to trigger constitutive activation of the integrin inside-out signaling molecule Rap1, resulting in translocation to the cell membrane (118). In transgenic mice expressing this JAK2-V617F protein in hematopoietic cells $\mathrm{JAK} 2^{+/ \mathrm{VF}}$ granulocytes showed increased binding of 9EG7 antibody, indicating the conformational activation of $\beta 1$ integrins. Moreover, increased expression of both $\beta 1$ and $\beta 2$ integrins was observed (118). The researchers also demonstrated the neutralizing anti- $\alpha 4$ (anti-VLA-4) and anti- $\beta_{2}$ integrin antibodies to suppress the pathologic thrombosis observed in JAK2 ${ }^{+/ \mathrm{VF}}$ mice. Additionally, the aberrant homing of $\mathrm{JAK} 2^{+/ \mathrm{VF}}$ leukocytes to the spleen was inhibited by the neutralizing anti- $\beta_{2}$ antibodies or alternatively pharmacologic inhibition of Rap1. These findings suggest that JAK2-V617F, a mutation commonly associated with myeloproliferative 
disorders (214), leads to integrin activation, promoting pathologic thrombosis and abnormal trafficking of leukocytes to the spleen (118).

Cancer-associated fibroblasts (CAFs) are the most abundant cells in a tumor (216). Attieh et al. found that CAFs isolated from the tumor of colon cancer patients secrete and assemble fibronectin more efficiently compared to noncancer-associated fibroblasts from the neighboring healthy tissue (25). Importantly, the amounts of secreted and assembled fibronectin correlated with the invasion index of the tumor. This study suggests mechanical signals to be important for CAF-mediated cancer cell invasion, as they induce fibronectin assembly. The authors propose that contractility of CAFs is necessary for downstream activation of the integrin- $\alpha v \beta 3$ and assembly of fibronectin puncta. $\alpha 5 \beta 1$ becomes critical only at later stages of fibronectin assembly. In summary, Attieh et al. (25) revealed that fibronectin-depositing CAFs enable cancer cells to invade the matrix and this process is independent of matrix metalloproteinases.

\section{LAD-III: Kindlin-3 is essential for proper integrin activation}

A rare autosomal leukocyte adhesion deficiency syndrome called LAD-III is characterized by severe bleeding and impaired adhesion of leukocytes to inflamed endothelia. A hallmark of this recessive disease is the impaired activation of $\beta 1, \beta 2$ and $\beta 3$ integrins on platelets and leukocytes (12).

Malinin et al. described a kindlin-3 point mutation causing serious bleeding, frequent infections and osteopetrosis at an early age (277). They found the symptoms to be caused by an integrin activation defect in hematopoietic cells, including platelets and leukocytes. The lymphocyte cell line established from the patient was phenotypically rescued by expression of wild-type kindlin-3, proving the association of the disease to the defective kindlin-3. The inactivation of kindlin-3 was caused by a point mutation creating a premature stop codon at the amino acid position 16. Importantly, all the clinical symptoms of the subjects were resolved by an allogenic bone marrow transplantation. Also another study by Svensson et al. (421) found a link between kindlin-3 and LAD-III, identifying two independent mutations causing decreased KINDLIN3 messenger RNA levels and loss of protein expression.

During the last years it has become evident that integrins are not only activated by talin, but also in coordination with kindlin binding to the second NxxY motif (335). As kindlin-3 is expressed exclusively in hematopoietic cells, it is clear now that LAD-III is caused by 
insufficient integrin activation due to the lacking kindlin contribution. Moser et al. 2008 (295) found kindlin-3 to interact with $\beta 1$ and $\beta 3$ integrin tails both in the presence and absence of talin-1, with the F3 subdomain of kindlin-3 being sufficient for this direct interaction. Later, Moser et al. 2009 (294) showed that kindlin-3 binds the cytoplasmic domain of $\beta 2$ integrin. They proposed kindlin- 3 to be essential for neutrophil adhesion and spreading on $\beta 2$ integrindependent ligands such as intercellular adhesion molecule-1 and the complement C3 activation product iC3b.

\section{Autosomal dominant polycystic kidney disease}

Integrins are highly important for the kidney development, and a comprehensive overview on the phenotypes associated with various integrin mutations in mouse models has been provided by Mathew et al. (282).

Autosomal dominant polycystic kidney disease (ADPKD) is a disorder involving the development of bilateral renal cysts, accumulation of extracellular matrix and tubulointerstitial fibrosis (440). The disease is caused by the mutation of the PKD1 or PKD2 genes encoding polycystin-1 (PC1) or polycystin-2 (PC2), respectively (184). Interestingly, the cystic epithelia express higher levels of integrins (246).

A recent study (246) showed that depletion of PC1 in immortalized renal collecting duct cells elevated the levels of integrin- $\beta 1$ and fibronectin and displayed increased integrin-mediated signaling in the presence of $\mathrm{Mn}^{2+}$ compared to wild-type cells. Conditional inactivation of integrin- $\beta 1$ in collecting ducts of mice resulted in a dramatic inhibition of $P k d l$-dependent cystogenesis with a concomitant suppression of fibrosis and preservation of normal renal function. These results suggest functional integrin- $\beta 1$ to be required for the early events leading to renal cystogenesis in ADPKD.

\section{Integrins and liver diseases}

Chronic injury in liver is characterized by intense production of collagens and other ECM components accompanied with their decelerated degradation, leading to net matrix accumulation (reviewed in (389)). Several clinical studies have found changes in integrin expression to be associated with chronic liver diseases. Nejjari et al. (306) studied patients with chronic hepatitis $\mathrm{C}$ and found an increase in $\beta 1$ labeling intensity in 83 out of 94 patients (88.2\%). Moreover, also expression of $\alpha 1, \alpha 5$ and $\alpha 6$ integrins were pronounced (306). Popov 
et al. (340) extracted total RNA from explant livers of patients undergoing liver transplantation. They found $\beta 6$ integrin mRNA transcript levels to be significantly elevated in patients with chronic hepatitis B and C, primary biliary cirrhosis, primary sclerosing cholangitis and alcoholinduced liver injury. This suggests integrin overexpression to be an etiology-independent factor associated with liver fibrosis.

\section{Atherosclerosis}

Atherosclerosis is a chronic inflammatory disease involving accumulation of lipids, cell debris and extracellular matrix proteins as well as monocyte-derived macrophages at the inflamed vascular wall. The M1/M2 macrophage ratio can be considered a determinant of plaque stability (reviewed in (325)). A study by Cho et al. revealed that M1 macrophages are exclusively found in plaques of symptomatic patients and elevated in unstable plaques (87). Aziz et al. (27) studied the role of $\alpha \mathrm{D} \beta 2$ integrin in atherosclerosis using both mouse and human samples. In this study the retention of macrophages was linked to a significant upregulation of integrin $\alpha \mathrm{D} \beta 2$ in $\mathrm{M} 1$ macrophages in vitro and in macrophages in atherosclerotic lesions. The findings suggest that $\alpha \mathrm{D} \beta 2$ contributes to the development of chronic inflammation via regulation of macrophage migration. Interestingly, our study focusing on human arterial plaques showed upregulation of integrin $\beta 2$, while all other studied integrins (ITGA1, ITGAV, ITGB3, ITGB5) were downregulated (315).

\section{Integrins and smoking}

Pulmonary emphysema, largely attributable to tobacco smoke exposure, is a worldwide challenge. Morris et al. observed elevated expression of matrix metalloproteinase 12 in mice lacking the integrin $\alpha v \beta 6$, that developed progressive spontaneous emphysema. The emphysema was prevented by transgenic $\beta 6$ integrin expression, which, however, was dependent on the ability of $\beta 6$ to bind and activate latent TGF- $\beta$. Importantly, the pathological characteristics of mice lacking $\alpha v \beta 6$ integrin resemble those observed in young cigarette smokers (310).

Overbeek et al. (319) studied the effect of cigarette smoke on neutrophil migration and $\beta_{2}$ integrin activation. CD11b-expressing neutrophils appeared in the lungs of mice after exposure to cigarette smoke for 5 days. To interpret this finding further, they exposed freshly isolated human neutrophils to cigarette smoke extract (CSE). CSE activated $\alpha \mathrm{M} \beta 2$ on the neutrophils, leading to firm adhesion to fibrinogen. In response to CSE the neutrophils transmigrated 
through endothelium via the activation of $\beta_{2}$-integrins, and the functional block of CD11b and CD18 decreased this transmigration.

Although e-cigarettes could be considered a "healthy" choice compared to traditional cigarettes, a recent study revealed that platelets from e-cigarette-exposed mice are hyperactive, show enhanced aggregation and granule secretion. Importantly, also these platelets showed increased activation of the $\alpha \operatorname{IIb} \beta 3$ integrin (351).

\section{Chapter 9: Integrins and TGF- $\beta$ activation at the onset of fibrosis}

In recent years cancer cells were demonstrated to cooperate extensively with cancer-associated fibroblasts, shaping the tumor microenvironment (67). These CAFs rearrange the tumorsurrounding ECM, increase its stiffness and promote cancer cell invasion $(25,67)$. This pathological ECM stiffening, called fibrosis, is not restricted to cancer, but rather constitutes a pathology of its own. In a simplified way fibrosis can be described as excessive scar formation. While scars preserve the mechanical integrity of the tissue, they fail to support normal organ function, such as gas exchange in the lung or the beating of the heart. Ultimately fibrosis can lead to organ failure and death and is estimated to contribute to more than $40 \%$ of deaths in the developed world (468). The onset of fibrosis is characterized by the transformation of cells into contractile myofibroblasts, mediated by signaling molecules of the transforming growth factor $\beta$ (TGF- $\beta$ ) family (190). Interestingly, cells do not secrete TGF- $\beta$ in a soluble form, but rather as a complex with the latency associated peptide (LAP). This LAP/TGF- $\beta$ complex is anchored to the ECM via latent TGF- $\beta$ binding protein (LTBP), preventing TGF- $\beta$ from binding to its receptor (190). The release of TGF- $\beta$ from this complex requires the binding of integrins to an RGD sequence in LAP and mechanical load on the ECM-(LAP/TGF- $\beta$ )-integrin axis (299). In fact, replacing the RGD sequence in LAP by RGE prevents integrin binding to LAP and in mice leads to a phenotype comparable to a complete TGF- $\beta$ knockout (481). This highlights the importance of integrin binding in the process of TGF- $\beta$ release and subsequent transformation of cells into myofibroblasts. For the same reasons, integrins are also interesting drug targets for the treatment of fibrosis, where complete TGF- $\beta$ inhibition causes too many adverse side effects (240). 
A survey of the literature suggests that the complete group of $\alpha \mathrm{V}$ integrins, including $\alpha \mathrm{V} \beta 1$, $\alpha \mathrm{V} \beta 3, \alpha \mathrm{V} \beta 5, \alpha \mathrm{V} \beta 6$ and $\alpha \mathrm{V} \beta 8$, binds to LAP. However, TGF- $\beta$ activation appears to be mediated only by $\alpha \mathrm{V} \beta 8$ integrin and especially $\alpha \mathrm{V} \beta 6$ integrin (368), while a recent work indicates that also $\alpha \mathrm{V} \beta 1$ integrin might be relevant (361). Whether these integrins compensate each other or whether they act in a tissue- and development-specific context remains to be explored. Dong and colleagues (113) published structural data for the binding of LAP/TGF- $\beta$ to $\alpha \mathrm{V} \beta 6$ integrin and revealed that the binding interface of $\alpha \mathrm{V} \beta 6$ integrin and LAP/TGF- $\beta$ is highly interdigitated and larger than other integrin-ligand interfaces. This spatial arrangement of integrin and ligand might help to ensure the proper alignment of the force with the TGF- $\beta$ activation axis, when mechanical load is applied to the complex. Additionally, the unusually large interface between $\alpha \mathrm{V} \beta 6$ integrin and LAP/TGF- $\beta$ might help to increase the mechanical stability of the integrin-ligand bond. It also explains why $\beta 3$ and $\beta 5$ integrins cannot be receptors for the LAP/TGF- $\beta$ complex. Thus, the binding of $\alpha \mathrm{V} \beta 6$ integrin and LAP/TGF- $\beta$ offers an example of a specific structural and molecular arrangement supporting biological functions with consequences for the complete organism.

The importance of integrins in fibrosis is not limited to TGF- $\beta$ activation. The interplay of physical and biochemical parameters in fibrosis increased the interest in this pathology as a promising in vivo example for the relevance of mechanobiology. Many healthy tissues have a stiffness (measured as Young's modulus $E$ ) below $5 \mathrm{kPa}$, but exceed this value during fibrosis. For example, lung tissue switches from $2 \mathrm{kPa}$ in a healthy state up to $17 \mathrm{kPa}$ in fibrosis, while liver stiffness increases from below $1 \mathrm{kPa}$ and less up to $12 \mathrm{kPa}$ and more (461).

As discussed in Chapter 6, mechanotransduction for many processes is regulated by an on/offmechanoswitch that is triggered around a substrate stiffness of $5 \mathrm{kPa}$. Therefore, fibrotic tissue stiffness above $5 \mathrm{kPa}$ might cause a constant on-switch of YAP/TAZ-dependent proliferation, contributing to the positive feedback loop of YAP activation and tissue stiffening (67).

\section{Chapter 10: Outlook and perspectives}

Starting with Abercrombie (1), the research of integrins and integrin-mediated adhesions has a history of 40 years. We are not aware of many other fields in biology that attracted so constant interest for such a long time. One reason might be the technical accessibility of integrin- 
mediated adhesions, making it an easy target for the newest microscopic techniques or proteomic studies. At the same time, integrins and cell matrix adhesions proved repetitively that they are not passive 'gluing' structures fixing cells to the ECM. Instead, they are tightly regulated, integrating many intracellular and extracellular signals to create very diverse cellular processes ranging from adhesion, migration or ECM organization to proliferation. The multitudes of direct integrin-adapter interactions in the cell (Table 1 and Figure 4) reflect these diverse integrin functions. This complexity of integrin-mediated signaling clearly justified the ongoing interest in cell adhesion over the years. Whether the current interest in integrins and cell adhesions will last for another 40 years is hard to predict. But we feel that there are plenty of open questions remaining:

\section{Integrin-mediated mechanosensing}

We highlighted already the relevance of mechanical integrin regulation (Chapter 6) additional to the established influence of biochemical signaling. This is currently a vibrating area of research, and we expect the mechanobiology of cell adhesions to also have a profound impact on the development of integrin-targeted therapies (Chapter 8). The increasing number of examples for mechanical regulation of integrin conformation and thereby integrin activation (Chapter 2) imply, that this mechanical regulation has to be considered in the development of drugs targeting cell adhesion. For example, a drug binding to only one integrin conformation or a drug leading to allosteric head-piece opening may be more difficult to use in the clinic than a drug that acts as an integrin antagonist, blocking the conformation of integrins in the extended closed conformation upon binding (5). Alternatively, mechanical forces on integrins can affect its conformation and by this the binding affinity of the drug. Such an example has been documented for imatinib (Gleevec), which suppressed c-Kit kinase activity when stimulated with a soluble Kit-ligand, but failed to inhibit c-Kit signaling mediated by a mechanically anchored Kit-ligand (424). We assume that mechanical forces on the ligand-bound c-Kit rendered the binding pocket unavailable for imatinib, in contrast to the situation with soluble Kit-ligand, where the competitive inhibitor imatinib could bind and inhibit, c-Kit. Other examples include bacterial adhesins, giving rise to fibronectin-binding peptides that recognize only relaxed fibronectin fibrils (20). Such a load-dependent binding might be disadvantageous in a therapy, limiting the activity of the drug or antibody. At the same time, it is clear that integrins are a promising and powerful drug target, with successful therapies for thrombosis and emerging therapies for multiple sclerosis and other immune system-related diseases existing (165). In fact, immune suppression by targeting $\alpha 4 \beta 1$ integrin (natalizumab) or $\alpha \mathrm{L} \beta 2$ 
integrin (efalizumab) is too effective, causing progressive multifocal leukoencephalopathy by activating the human polyomavirus JVC in some patients (276). However, conformationand/or mechanical load-specific therapies might one day offer a less toxic, but more selective approach in therapies targeting cell adhesion.

\section{Integrins and therapies}

The safety and ultimate clinical success of integrin-targeting drugs remains still to be evaluated (Chapter 8). Nevertheless, it is indisputable that the failure of the $\alpha \mathrm{V} \beta 3$ integrin inhibitor cilengitide in phase III trials (including Merck giving up on further trials for this inhibitor) dampened the enthusiasm about chemotherapies targeting integrins. Are there more general, fundamental problems with drugs targeting integrins? Above, we mentioned the influence of mechanical forces on the integrin-ligand binding pocket and potential consequences for drug development. However, we also tried to raise awareness for the specificity of integrins, their ligands, and their adapters throughout this review. In other words, we might just not know enough about integrins for a faithful generalization. Structure-function relationship (Chapter 2), RIAM-dependent vs. -independent integrin activation (Chapter 3), reticular adhesions vs. focal adhesions (Chapter 3), or the relevance of $\alpha \mathrm{V} \beta 1$ integrin in fibrosis (Chapter 9) are examples where established findings were recently challenged. Kindlin as integrin activator is much less understood than talin, kank is still emerging as potentially important adapter, and all of them have isoforms that withstood evolutionary selection while we tend to ignore them to keep things simple. But maybe we still have to add more trees before we can clearly see the forest. In the end, integrin targeting therapies are most successful in intensively studied systems such as platelets and integrins in the immune system.

\section{Cell adhesion and metabolism}

The research of metabolism seems to expand very rapidly at the moment, and it is linked to an increasing number of topics including integrin research and the interplay between cell metabolism and cell adhesion. This is strikingly demonstrated by the effects of diabetes on ECM and integrin organization (21). Interestingly, the metabolic sensors mTOR (355) and AMPK (156) are both shown to act via tensins on fibrillar adhesions and endocytosis, thus organizing the ECM (Chapter 3). This link between integrin-dependent ECM organization and metabolism might also explain the correlation of fibrosis (Chapter 9) and obesity. Moreover, there are first indications that integrin and integrin adapters are targets of metabolism- 
dependent posttranslational modifications like acetylation $(89,460)$, potentially establishing a close link between metabolism and cell adhesion.

\section{Funding sources and acknowledgements}

The authors are grateful to funding by the Swiss National Science Foundation, the Swiss Cancer League, the Swiss Society for Muscle Research to BWH, as well as grateful to the Academy of Finland which supported this research via grants 290506, 273192 and 136288 to VPH. We thank Dr. Steffi Goffart (University of Eastern Finland) for reading the manuscript and for her helpful comments. The authors also thank the current and previous group members, colleagues and coauthors for fruitful discussions and their significant contributions.

\section{References}

1. Abercrombie M. Fibroblasts. J Clin Pathol Suppl (R Coll Pathol) 12: 1-6, 1978.

2. Abercrombie M, Heaysman JE, Pegrum SM. The locomotion of fibroblasts in culture. IV. Electron microscopy of the leading lamella. Exp Cell Res 67: 359-67, 1971.

3. Adair BD, Xiong J-P, Maddock C, Goodman SL, Arnaout MA, Yeager M. Threedimensional EM structure of the ectodomain of integrin $\alpha \mathrm{V} \beta 3$ in a complex with fibronectin. $J$ Cell Biol 168: 1109-1118, 2005.

4. Adhikari AS, Chai J, Dunn AR. Mechanical load induces a 100-fold increase in the rate of collagen proteolysis by MMP-1. J Am Chem Soc 133: 1686-9, 2011.

5. Van Agthoven JF, Xiong J-P, Alonso JL, Rui X, Adair BD, Goodman SL, Arnaout MA. Structural basis for pure antagonism of integrin $\alpha \mathrm{V} \beta 3$ by a high-affinity form of fibronectin. Nat Struct Mol Biol 21: 383-8, 2014.

6. Ahmed N, Niu J, Dorahy DJ, Gu X, Andrews S, Meldrum CJ, Scott RJ, Baker MS, Macreadie IG, Agrez M V. Direct integrin $\alpha \mathrm{v} \beta 6$-ERK binding: implications for tumour growth. Oncogene 21: 1370-80, 2002.

7. Akiyama SK, Yamada KM. Synthetic peptides competitively inhibit both direct binding to fibroblasts and functional biological assays for the purified cell-binding domain of fibronectin. J Biol Chem 260: 10402-5, 1985.

8. Akiyama SK, Yamada SS, Yamada KM. Characterization of a 140-kD avian cell surface antigen as a fibronectin-binding molecule. J Cell Biol 102: 442-8, 1986.

9. Alahari SK, Nasrallah H. A membrane proximal region of the integrin $\alpha 5$ subunit is important for its interaction with nischarin. Biochem J 377: 449-57, 2004.

10. Alahari SK, Reddig PJ, Juliano RL. The integrin-binding protein Nischarin regulates cell migration by inhibiting PAK. EMBO J 23: 2777-2788, 2004.

11. Alanko J, Mai A, Jacquemet G, Schauer K, Kaukonen R, Saari M, Goud B, Ivaska J. 
Integrin endosomal signalling suppresses anoikis. Nat Cell Biol 17: 1412-21, 2015.

12. Alon R, Etzioni A. LAD-III, a novel group of leukocyte integrin activation deficiencies. Trends Immunol 24: 561-6, 2003.

13. Alrehani N, Pradhan S, Khatlani T, Kailasam L, Vijayan KV. Distinct roles for the $\alpha, \beta$ and $\gamma 1$ isoforms of protein phosphatase 1 in the outside-in $\alpha \operatorname{IIb} \beta 3$ integrin signalling-dependent functions. Thromb Haemost 109: 118-26, 2013.

14. Anthis NJ, Haling JR, Oxley CL, Memo M, Wegener KL, Lim CJ, Ginsberg MH, Campbell ID. B integrin tyrosine phosphorylation is a conserved mechanism for regulating talin-induced integrin activation. J Biol Chem 284: 36700-10, 2009.

15. Anthis NJ, Wegener KL, Critchley DR, Campbell ID. Structural diversity in integrin/talin interactions. Structure 18: 1654-66, 2010.

16. Anthis NJ, Wegener KL, Ye F, Kim C, Goult BT, Lowe ED, Vakonakis I, Bate N, Critchley DR, Ginsberg MH, Campbell ID. The structure of an integrin/talin complex reveals the basis of inside-out signal transduction. EMBO J 28: 3623-3632, 2009.

17. Arias-Salgado EG, Haj F, Dubois C, Moran B, Kasirer-Friede A, Furie BC, Furie B, Neel BG, Shattil SJ. PTP-1B is an essential positive regulator of platelet integrin signaling. $J$ Cell Biol 170: 837-45, 2005.

18. Arias-Salgado EG, Lizano S, Sarkar S, Brugge JS, Ginsberg MH, Shattil SJ. Src kinase activation by direct interaction with the integrin cytoplasmic domain. Proc Natl Acad Sci 100: 13298-13302, 2003

19. Arias-Salgado EG, Lizano S, Shattil SJ, Ginsberg MH. Specification of the direction of adhesive signaling by the integrin $\beta$ cytoplasmic domain. J Biol Chem 280: 29699-707, 2005.

20. Arnoldini S, Moscaroli A, Chabria M, Hilbert M, Hertig S, Schibli R, Béhé M, Vogel V. Novel peptide probes to assess the tensional state of fibronectin fibers in cancer. Nat Commun 8: 1793, 2017.

21. Arous C, Wehrle-Haller B. Role and impact of the extracellular matrix on integrin-mediated pancreatic $\beta$-cell functions. Biol cell 109: 223-237, 2017.

22. Arroyo AG, Sánchez-Mateos P, Campanero MR, Martín-Padura I, Dejana E, SánchezMadrid F. Regulation of the VLA integrin-ligand interactions through the $\beta 1$ subunit. $J$ Cell Biol 117: 659-70, 1992.

23. Arthos J, Cicala C, Martinelli E, Macleod K, Van Ryk D, Wei D, Xiao Z, Veenstra TD, Conrad TP, Lempicki RA, McLaughlin S, Pascuccio M, Gopaul R, McNally J, Cruz CC, Censoplano N, Chung E, Reitano KN, Kottilil S, Goode DJ, Fauci AS. HIV-1 envelope protein binds to and signals through integrin $\alpha 4 \beta 7$, the gut mucosal homing receptor for peripheral T cells. Nat Immunol 9: 301-9, 2008.

24. Atherton P, Stutchbury B, Wang D-Y, Jethwa D, Tsang R, Meiler-Rodriguez E, Wang P, Bate N, Zent R, Barsukov IL, Goult BT, Critchley DR, Ballestrem C. Vinculin controls talin engagement with the actomyosin machinery. Nat Commun 6: 10038, 2015.

25. Attieh Y, Clark AG, Grass C, Richon S, Pocard M, Mariani P, Elkhatib N, Betz T, Gurchenkov B, Vignjevic DM. Cancer-associated fibroblasts lead tumor invasion through 
integrin- $\beta 3$-dependent fibronectin assembly. J Cell Biol 216: 3509-3520, 2017.

26. Austen K, Ringer P, Mehlich A, Chrostek-Grashoff A, Kluger C, Klingner C, Sabass B, Zent R, Rief M, Grashoff C. Extracellular rigidity sensing by talin isoform-specific mechanical linkages. Nat Cell Biol 17: 1597-606, 2015.

27. Aziz MH, Cui K, Das M, Brown KE, Ardell CL, Febbraio M, Pluskota E, Han J, Wu H, Ballantyne CM, Smith JD, Cathcart MK, Yakubenko VP. The Upregulation of Integrin $\alpha \mathrm{D} \beta 2(\mathrm{CD} 11 \mathrm{~d} / \mathrm{CD} 18)$ on Inflammatory Macrophages Promotes Macrophage Retention in Vascular Lesions and Development of Atherosclerosis. J Immunol 198: 4855-4867, 2017.

28. El azreq M-A, Bourgoin SG. Cytohesin-1 regulates human blood neutrophil adhesion to endothelial cells through $\beta 2$ integrin activation. Mol Immunol 48: 1408-1416, 2011.

29. El Azreq M-A, Garceau V, Bourgoin SG. Cytohesin-1 regulates fMLF-mediated activation and functions of the $\beta_{2}$ integrin Mac-1 in human neutrophils. J Leukoc Biol 89: 823-836, 2011.

30. Bachir AI, Zareno J, Moissoglu K, Plow EF, Gratton E, Horwitz AR. Integrin-Associated Complexes Form Hierarchically with Variable Stoichiometry in Nascent Adhesions. Curr Biol 24: 1845-1853, 2014.

31. Bachmann M, Schäfer M, Mykuliak V, Ripamonti M, Heiser L, Weißenbruch K, Krübel S, Franz CM, Hytönen V, Wehrle-Haller B, Bastmeyer M. Ligand binding promiscuity of $\alpha \mathrm{V} \beta 3$ integrin is enlarged in response to mechanical force. bioRxiv ( November 20, 2018). doi: $10.1101 / 200493$.

32. Bai M, Pang X, Lou J, Zhou Q, Zhang K, Ma J, Li J, Sun F, Hsu VW. Mechanistic Insights into Regulated Cargo Binding by ACAP1 Protein. J Biol Chem 287: 28675-28685, 2012.

33. Bajic G, Yatime L, Sim RB, Vorup-Jensen T, Andersen GR. Structural insight on the recognition of surface-bound opsonins by the integrin I domain of complement receptor 3. Proc Natl Acad Sci U S A 110: 16426-31, 2013.

34. Balcioglu HE, van Hoorn H, Donato DM, Schmidt T, Danen EHJ. The integrin expression profile modulates orientation and dynamics of force transmission at cell-matrix adhesions. $J$ Cell Sci 128: 1316-26, 2015.

35. Ballestrem C, Hinz B, Imhof BA, Wehrle-Haller B. Marching at the front and dragging behind. J Cell Biol 155: 1319-1332, 2001.

36. Bandyopadhyay A, Rothschild G, Kim S, Calderwood DA, Raghavan S. Functional differences between kindlin-1 and kindlin-2 in keratinocytes. J Cell Sci 125: 2172-84, 2012.

37. Barry WT, Boudignon-Proudhon C, Shock DD, McFadden A, Weiss JM, Sondek J, Parise L V. Molecular basis of CIB binding to the integrin a IIb cytoplasmic domain. J Biol Chem 277: 28877-83, 2002.

38. Baschieri F, Dayot S, Elkhatib N, Ly N, Capmany A, Schauer K, Betz T, Vignjevic DM, Poincloux R, Montagnac G. Frustrated endocytosis controls contractility-independent mechanotransduction at clathrin-coated structures. Nat Commun 9: 3825, 2018.

39. Berditchevski F, Odintsova E, Sawada S, Gilbert E. Expression of the palmitoylationdeficient CD151 weakens the association of $\alpha 3 \beta 1$ integrin with the tetraspanin-enriched microdomains and affects integrin-dependent signaling. J Biol Chem 277: 36991-7000, 2002. 
40. Bergelson JM, Chan BM, Finberg RW, Hemler ME. The integrin VLA-2 binds echovirus 1 and extracellular matrix ligands by different mechanisms. J Clin Invest 92: 232-239, 1993.

41. Bergelson JM, Shepley MP, Chan BM, Hemler ME, Finberg RW. Identification of the integrin VLA-2 as a receptor for echovirus 1. Science 255: 1718-20, 1992.

42. Berman HM, Westbrook J, Feng Z, Gilliland G, Bhat TN, Weissig H, Shindyalov IN, Bourne PE. The Protein Data Bank. Nucleic Acids Res 28: 235-242, 2000.

43. Bertotti A, Comoglio PM, Trusolino L. B4 integrin activates a Shp2-Src signaling pathway that sustains HGF-induced anchorage-independent growth. J Cell Biol 175: 993-1003, 2006.

44. Biffo S, Sanvito F, Costa S, Preve L, Pignatelli R, Spinardi L, Marchisio PC. Isolation of a novel $\beta 4$ integrin-binding protein (p27(BBP)) highly expressed in epithelial cells. J Biol Chem 272: 30314-21, 1997.

45. Bledzka K, Bialkowska K, Nie H, Qin J, Byzova T, Wu C, Plow EF, Ma Y-Q. Tyrosine phosphorylation of integrin $\beta 3$ regulates kindlin-2 binding and integrin activation. $J$ Biol Chem 285: 30370-4, 2010.

46. Bledzka K, Liu J, Xu Z, Perera D, Yadav SP, Bialkowska K, Qin J, Ma Y-Q, Plow EF. Spatial Coordination of Kindlin-2 with Talin Head Domain in Interaction with Integrin $\beta$ Cytoplasmic Tails. J Biol Chem 287: 24585-24594, 2012.

47. Boisguerin P, Leben R, Ay B, Radziwill G, Moelling K, Dong L, Volkmer-Engert R. An improved method for the synthesis of cellulose membrane-bound peptides with free $\mathrm{C}$ termini is useful for PDZ domain binding studies. Chem Biol 11: 449-59, 2004.

48. Bonet R, Vakonakis I, Campbell ID. Characterization of 14-3-3- $\zeta$ Interactions with integrin tails. J Mol Biol 425: 3060-72, 2013.

49. Böttcher RT, Lange A, Fässler R. How ILK and kindlins cooperate to orchestrate integrin signaling. Curr Opin Cell Biol 21: 670-5, 2009.

50. Böttcher RT, Stremmel C, Meves A, Meyer H, Widmaier M, Tseng H-Y, Fässler R.

Sorting nexin 17 prevents lysosomal degradation of $\beta 1$ integrins by binding to the $\beta 1$-integrin tail. Nat Cell Biol 14: 584-592, 2012.

51. Böttcher RT, Veelders M, Rombaut P, Faix J, Theodosiou M, Stradal TE, Rottner K, Zent R, Herzog F, Fässler R. Kindlin-2 recruits paxillin and Arp2/3 to promote membrane protrusions during initial cell spreading. J Cell Biol 216: 3785-3798, 2017.

52. Bouchet BP, Gough RE, Ammon Y-C, van de W, Post H, Jacquemet G, Altelaar AFM, Heck AJR, Goult BT, Akhmanova A. Talin-KANK1 interaction controls the recruitment of cortical microtubule stabilizing complexes to focal adhesions. Elife 5: e18124, 2016.

53. Bouchet BP, Gough RE, Ammon Y-C, van de Willige D, Post H, Jacquemet G, Altelaar AM, Heck AJ, Goult BT, Akhmanova A. Talin-KANK1 interaction controls the recruitment of cortical microtubule stabilizing complexes to focal adhesions. Elife 5, 2016.

54. Bouvard D, Pouwels J, De Franceschi N, Ivaska J. Integrin inactivators: balancing cellular functions in vitro and in vivo. Nat Rev Mol Cell Biol 14: 430-442, 2013.

55. Bouvard D, Vignoud L, Dupé-Manet S, Abed N, Fournier H-N, Vincent-Monegat C, Retta SF, Fassler R, Block MR. Disruption of focal adhesions by integrin cytoplasmic 
domain-associated protein-1 a. J Biol Chem 278: 6567-74, 2003.

56. Brakebusch C, Grose R, Quondamatteo F, Ramirez A, Jorcano JL, Pirro A, Svensson M, Herken R, Sasaki T, Timpl R, Werner S, Fässler R. Skin and hair follicle integrity is crucially dependent on $\beta 1$ integrin expression on keratinocytes. EMBO J 19: 3990-4003, 2000.

57. Brancaccio M, Guazzone S, Menini N, Sibona E, Hirsch E, De Andrea M, Rocchi M, Altruda F, Tarone G, Silengo L. Melusin is a new muscle-specific interactor for $\beta(1)$ integrin cytoplasmic domain. [Online]. J Biol Chem 274: 29282-8, 1999. http://www.ncbi.nlm.nih.gov/pubmed/10506186 [11 Mar. 2019].

58. Bromberger T, Klapproth S, Rohwedder I, Zhu L, Mittmann L, Reichel CA, Sperandio M, Qin J, Moser M. Direct Rap1/Talin1 interaction regulates platelet and neutrophil integrin activity in mice. Blood 132: 2754-2762, 2018.

59. Brooks PC, Clark RA, Cheresh DA. Requirement of vascular integrin $\alpha$ v $\beta 3$ for angiogenesis. Science 264: 569-71, 1994.

60. Brophy TM, Raab M, Daxecker H, Culligan KG, Lehmann I, Chubb AJ, Treumann A, Moran N. RN181, a novel ubiquitin E3 ligase that interacts with the KVGFFKR motif of platelet integrin aIIbß3. Biochem Biophys Res Commun 369: 1088-1093, 2008.

61. Brunner M, Millon-Frémillon A, Chevalier G, Nakchbandi IA, Mosher D, Block MR, Albigès-Rizo C, Bouvard D. Osteoblast mineralization requires $\beta 1$ integrin/ICAP-1-dependent fibronectin deposition. J Cell Biol 194: 307-22, 2011.

62. Bunch TA. Integrin $\alpha \operatorname{IIb} \beta 3$ activation in Chinese hamster ovary cells and platelets increases clustering rather than affinity. J Biol Chem 285: 1841-9, 2010.

63. Byron A, Humphries JD, Bass MD, Knight D, Humphries MJ. Proteomic analysis of integrin adhesion complexes. Sci Signal 4: pt2, 2011.

64. Calderwood DA. The Rap1-RIAM pathway prefers $\beta 2$ integrins. Blood 126: 2658-9, 2015.

65. Calderwood DA, Fujioka Y, de Pereda JM, García-Alvarez B, Nakamoto T, Margolis B, McGlade CJ, Liddington RC, Ginsberg MH. Integrin $\beta$ cytoplasmic domain interactions with phosphotyrosine-binding domains: a structural prototype for diversity in integrin signaling. Proc Natl Acad Sci U S A 100: 2272-7, 2003.

66. Calderwood DA, Zent R, Grant R, Rees DJG, Hynes RO, Ginsberg MH. The Talin Head Domain Binds to Integrin $B$ Subunit Cytoplasmic Tails and Regulates Integrin Activation. $J$ Biol Chem 274: 28071-28074, 1999.

67. Calvo F, Ege N, Grande-Garcia A, Hooper S, Jenkins RP, Chaudhry SI, Harrington K, Williamson P, Moeendarbary E, Charras G, Sahai E. Mechanotransduction and YAPdependent matrix remodelling is required for the generation and maintenance of cancerassociated fibroblasts. Nat Cell Biol 15: 637-46, 2013.

68. Camp D, Haage A, Solianova V, Castle WM, Xu QA, Lostchuck E, Goult BT, Tanentzapf G. Direct binding of Talin to Rap1 is required for cell-ECM adhesion in Drosophila. J Cell Sci 131: jcs225144, 2018.

69. Cao L, Nicosia J, Larouche J, Zhang Y, Bachman H, Brown AC, Holmgren L, Barker TH. Detection of an Integrin-Binding Mechanoswitch within Fibronectin during Tissue 
Formation and Fibrosis. ACS Nano 11: 7110-7117, 2017.

70. Cardó-Vila M, Arap W, Pasqualini R. A v $\beta 5$ integrin-dependent programmed cell death triggered by a peptide mimic of annexin V. Mol Cell 11: 1151-62, 2003.

71. Carisey A, Ballestrem C. Vinculin, an adapter protein in control of cell adhesion signalling. Eur J Cell Biol 90: 157-63, 2011.

72. Caswell PT, Spence HJ, Parsons M, White DP, Clark K, Cheng KW, Mills GB, Humphries MJ, Messent AJ, Anderson KI, McCaffrey MW, Ozanne BW, Norman JC. Rab25 Associates with $\alpha 5 \beta 1$ Integrin to Promote Invasive Migration in 3D Microenvironments. Dev Cell 13: 496-510, 2007.

73. Chan CE, Odde DJ. Traction dynamics of filopodia on compliant substrates. Science 322: 1687-91, 2008.

74. Chang DD, Wong C, Smith H, Liu J. ICAP-1, a novel $\beta 1$ integrin cytoplasmic domainassociated protein, binds to a conserved and functionally important NPXY sequence motif of ß1 integrin. J Cell Biol 138: 1149-57, 1997.

75. Changede R, Xu X, Margadant F, Sheetz MP. Nascent Integrin Adhesions Form on All Matrix Rigidities after Integrin Activation. Dev Cell 35: 614-621, 2015.

76. Chao W-T, Ashcroft F, Daquinag AC, Vadakkan T, Wei Z, Zhang P, Dickinson ME, Kunz J. Type I Phosphatidylinositol Phosphate Kinase B Regulates Focal Adhesion Disassembly by Promoting 1 Integrin Endocytosis. Mol Cell Biol 30: 4463-4479, 2010.

77. Chao W-T, Kunz J. Focal adhesion disassembly requires clathrin-dependent endocytosis of integrins. FEBS Lett 583: 1337-1343, 2009.

78. Chatterjee D, D'Souza A, Zhang Y, Bin W, Tan S-M, Bhattacharjya S. Interaction

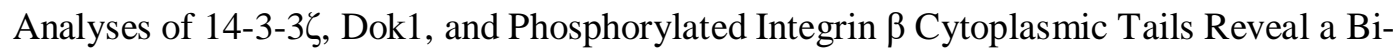
molecular Switch in Integrin Regulation. J Mol Biol 430: 4419-4430, 2018.

79. Chen CS. Mechanotransduction - a field pulling together? J Cell Sci 121: 3285-92, 2008.

80. Chen L, Hughes RA, Baines AJ, Conboy J, Mohandas N, An X. Protein 4.1R regulates cell adhesion, spreading, migration and motility of mouse keratinocytes by modulating surface expression of $\beta 1$ integrin. J Cell Sci 124: 2478-87, 2011.

81. Chen L, Wang T, Wang Y, Zhang J, Qi Y, Weng H, Kang Q, Guo X, Baines AJ, Mohandas N, An X. Protein 4.1G Regulates Cell Adhesion, Spreading, and Migration of Mouse Embryonic Fibroblasts through the $\beta 1$ Integrin Pathway. J Biol Chem 291: 2170-80, 2016.

82. Chen Y, Ju L, Rushdi M, Ge C, Zhu C. Receptor-mediated cell mechanosensing. Mol Biol Cell 28: 3134-3155, 2017.

83. Chen Y, Lee H, Tong H, Schwartz M, Zhu C. Force regulated conformational change of integrin aVß3. Matrix Biol 60-61: 70-85, 2017.

84. Chen Z, Oh D, Biswas KH, Yu C-H, Zaidel-Bar R, Groves JT. Spatially modulated ephrinA1:EphA2 signaling increases local contractility and global focal adhesion dynamics to promote cell motility. Proc Natl Acad Sci U S A 115: E5696-E5705, 2018. 
85. Cheresh DA, Berliner SA, Vicente V, Ruggeri ZM. Recognition of distinct adhesive sites on fibrinogen by related integrins on platelets and endothelial cells. Cell 58: 945-53, 1989.

86. Chiaretti S, de Curtis I. Role of Liprins in the Regulation of Tumor Cell Motility and Invasion. Curr Cancer Drug Targets 16: 238-248, 2016.

87. Cho KY, Miyoshi H, Kuroda S, Yasuda H, Kamiyama K, Nakagawara J, Takigami M, Kondo T, Atsumi T. The Phenotype of Infiltrating Macrophages Influences Arteriosclerotic Plaque Vulnerability in the Carotid Artery. J Stroke Cerebrovasc Dis 22: 910-918, 2013.

88. Choi CK, Vicente-Manzanares M, Zareno J, Whitmore LA, Mogilner A, Horwitz AR. Actin and $\alpha$-actinin orchestrate the assembly and maturation of nascent adhesions in a myosin II motor-independent manner. Nat Cell Biol 10: 1039-1050, 2008.

89. Choudhary C, Kumar C, Gnad F, Nielsen ML, Rehman M, Walther TC, Olsen J V, Mann M. Lysine acetylation targets protein complexes and co-regulates major cellular functions. Science 325: 834-40, 2009.

90. Cluzel C, Saltel F, Lussi J, Paulhe F, Imhof BA, Wehrle-Haller B. The mechanisms and dynamics of avß3 integrin clustering in living cells. J Cell Biol 171: 383-392, 2005.

91. Coller BS, Peerschke EI, Scudder LE, Sullivan CA. A murine monoclonal antibody that completely blocks the binding of fibrinogen to platelets produces a thrombasthenic-like state in normal platelets and binds to glycoproteins IIb and/or IIIa. [Online]. J Clin Invest 72: 325-38, 1983. http://www.ncbi.nlm.nih.gov/pubmed/6308050 [8 Mar. 2019].

92. Cormier A, Campbell MG, Ito S, Wu S, Lou J, Marks J, Baron JL, Nishimura SL, Cheng Y. Cryo-EM structure of the $\alpha \mathrm{v} \beta 8$ integrin reveals a mechanism for stabilizing integrin extension. Nat Struct Mol Biol 25: 698-704, 2018.

93. Cowan KJ, Law DA, Phillips DR. Identification of shc as the primary protein binding to the tyrosine-phosphorylated $\beta 3$ subunit of $\alpha \operatorname{IIb} \beta 3$ during outside-in integrin platelet signaling. $J$ Biol Chem 275: 36423-9, 2000.

94. Crest J, Diz-Muñoz A, Chen D-Y, Fletcher DA, Bilder D. Organ sculpting by patterned extracellular matrix stiffness. Elife 6, 2017.

95. Cukierman E, Pankov R, Stevens DR, Yamada KM. Taking Cell-Matrix Adhesions to the Third Dimension. Science (80- ) 294: 1708-1712, 2001.

96. Curtis AS. The mechanism of adhesion of cells to glass. A study by interference reflection microscopy. J Cell Biol 20: 199-215, 1964.

97. Damsky CH, Knudsen KA, Bradley D, Buck CA, Horwitz AF. Distribution of the cell substratum attachment (CSAT) antigen on myogenic and fibroblastic cells in culture. $J$ Cell Biol 100: 1528-39, 1985.

98. Danen EHJ, Sonneveld P, Brakebusch C, Fässler R, Sonnenberg A. The fibronectinbinding integrins $\alpha 5 \beta 1$ and $\alpha v \beta 3$ differentially modulate RhoA-GTP loading, organization of cell matrix adhesions, and fibronectin fibrillogenesis. J Cell Biol 159: 1071-1086, 2002.

99. Dans M, Gagnoux-Palacios L, Blaikie P, Klein S, Mariotti A, Giancotti FG. Tyrosine phosphorylation of the $\beta 4$ integrin cytoplasmic domain mediates Shc signaling to extracellular signal-regulated kinase and antagonizes formation of hemidesmosomes. J Biol Chem 276: 
1494-502, 2001.

100. Das A, Fischer RS, Pan D, Waterman CM. YAP Nuclear Localization in the Absence of Cell-Cell Contact Is Mediated by a Filamentous Actin-dependent, Myosin II- and PhosphoYAP-independent Pathway during Extracellular Matrix Mechanosensing. J Biol Chem 291: 6096-110, 2016.

101. De S, Razorenova O, McCabe NP, O'Toole T, Qin J, Byzova T V. VEGF-integrin interplay controls tumor growth and vascularization. Proc Natl Acad Sci 102: 7589-7594, 2005.

102. Deakin NO, Bass MD, Warwood S, Schoelermann J, Mostafavi-Pour Z, Knight D, Ballestrem C, Humphries MJ. An integrin- 4-14-3-3 -paxillin ternary complex mediates localised Cdc42 activity and accelerates cell migration. J Cell Sci 122: 1654-1664, 2009.

103. Deakin NO, Turner CE. Paxillin comes of age. J Cell Sci 121: 2435-44, 2008.

104. Debrand E, Conti FJ, Bate N, Spence L, Mazzeo D, Pritchard CA, Monkley SJ, Critchley DR. Mice carrying a complete deletion of the talin2 coding sequence are viable and fertile. Biochem Biophys Res Commun 426: 190-195, 2012.

105. Denti S, Sirri A, Cheli A, Rogge L, Innamorati G, Putignano S, Fabbri M, Pardi R, Bianchi E. RanBPM is a phosphoprotein that associates with the plasma membrane and interacts with the integrin LFA-1. J Biol Chem 279: 13027-34, 2004.

106. Dersch P, Isberg RR. A region of the Yersinia pseudotuberculosis invasin protein enhances integrin-mediated uptake into mammalian cells and promotes self-association. EMBO J 18: 1199-213, 1999.

107. Desgrosellier JS, Cheresh DA. Integrins in cancer: biological implications and therapeutic opportunities. Nat Rev Cancer 10: 9-22, 2010.

108. Deshmukh L, Gorbatyuk V, Vinogradova O. Integrin $\{\beta\} 3$ phosphorylation dictates its complex with the Shc phosphotyrosine-binding (PTB) domain. J Biol Chem 285: 34875-84, 2010.

109. Deshmukh L, Tyukhtenko S, Liu J, Fox JEB, Qin J, Vinogradova O. Structural Insight into the Interaction between Platelet Integrin $\alpha_{\text {IIb }} \beta_{3}$ and Cytoskeletal Protein Skelemin. J Biol Chem 282: 32349-32356, 2007.

110. van Dinther-Janssen AC, Horst E, Koopman G, Newmann W, Scheper RJ, Meijer CJ, Pals ST. The VLA-4/VCAM-1 pathway is involved in lymphocyte adhesion to endothelium in rheumatoid synovium. [Online]. J Immunol 147: 4207-10, 1991. http://www.ncbi.nlm.nih.gov/pubmed/1721640 [31 Oct. 2018].

111. DiPersio CM, Hodivala-Dilke KM, Jaenisch R, Kreidberg JA, Hynes RO. $\alpha 3 \beta 1$ Integrin is required for normal development of the epidermal basement membrane. J Cell Biol 137: 729_ 42, 1997.

112. DiPersio CM, van der Neut R, Georges-Labouesse E, Kreidberg JA, Sonnenberg A, Hynes RO. $\alpha 3 \beta 1$ and $\alpha 6 \beta 4$ integrin receptors for laminin- 5 are not essential for epidermal morphogenesis and homeostasis during skin development. J Cell Sci 113 ( Pt 1: 3051-62, 2000.

113. Dong X, Zhao B, Iacob RE, Zhu J, Koksal AC, Lu C, Engen JR, Springer TA. Force 
interacts with macromolecular structure in activation of TGF- $\beta$. Nature 542: 55-59, 2017.

114. Dowling J, Yu QC, Fuchs E. B4 integrin is required for hemidesmosome formation, cell adhesion and cell survival. J Cell Biol 134: 559-72, 1996.

115. Dumbauld DW, García AJ. A helping hand: How vinculin contributes to cell-matrix and cellcell force transfer. Cell Adh Migr 8: 550-7, 2014.

116. Dupont S. Role of YAP/TAZ in cell-matrix adhesion-mediated signalling and mechanotransduction. Exp Cell Res 343: 42-53, 2016.

117. Dustin ML, Springer TA. Lymphocyte function-associated antigen-1 (LFA-1) interaction with intercellular adhesion molecule-1 (ICAM-1) is one of at least three mechanisms for lymphocyte adhesion to cultured endothelial cells. J Cell Biol 107: 321-31, 1988.

118. Edelmann B, Gupta N, Schnoeder TM, Oelschlegel AM, Shahzad K, Goldschmidt J, Philipsen L, Weinert S, Ghosh A, Saalfeld FC, Nimmagadda SC, Müller P, BraunDullaeus R, Mohr J, Wolleschak D, Kliche S, Amthauer H, Heidel FH, Schraven B, Isermann B, Müller AJ, Fischer T. JAK2-V617F promotes venous thrombosis through $\beta 1 / \beta 2$ integrin activation. $J$ Clin Invest 128: 4359-4371, 2018.

119. Egervari K, Potter G, Guzman-Hernandez ML, Salmon P, Soto-Ribeiro M, Kastberger B, Balla T, Wehrle-Haller B, Kiss JZ. Astrocytes spatially restrict VEGF signaling by polarized secretion and incorporation of VEGF into the actively assembling extracellular matrix. Glia 64: 440-456, 2016.

120. Eliceiri BP, Puente XS, Hood JD, Stupack DG, Schlaepfer DD, Huang XZ, Sheppard D, Cheresh DA. Src-mediated coupling of focal adhesion kinase to integrin $\alpha(v) \beta 5$ in vascular endothelial growth factor signaling. J Cell Biol 157: 149-60, 2002.

121. Elosegui-Artola A, Andreu I, Beedle AEM, Lezamiz A, Uroz M, Kosmalska AJ, Oria R, Kechagia JZ, Rico-Lastres P, Le Roux A-L, Shanahan CM, Trepat X, Navajas D, GarciaManyes S, Roca-Cusachs P. Force Triggers YAP Nuclear Entry by Regulating Transport across Nuclear Pores. Cell 171: 1397-1410.e14, 2017.

122. Elosegui-Artola A, Oria R, Chen Y, Kosmalska A, Pérez-González C, Castro N, Zhu C, Trepat X, Roca-Cusachs P. Mechanical regulation of a molecular clutch defines force transmission and transduction in response to matrix rigidity. Nat Cell Biol 18: 540-8, 2016.

123. Elosegui-Artola A, Trepat X, Roca-Cusachs P. Control of Mechanotransduction by Molecular Clutch Dynamics. Trends Cell Biol 28: 356-367, 2018.

124. Emsley J, Knight CG, Farndale RW, Barnes MJ, Liddington RC. Structural basis of collagen recognition by integrin $\alpha 2 \beta 1$. Cell 101: 47-56, 2000.

125. Eng ET, Smagghe BJ, Walz T, Springer TA. Intact $\alpha_{\text {Iть }} \beta_{3}$ Integrin Is Extended after Activation as Measured by Solution X-ray Scattering and Electron Microscopy. J Biol Chem 286: 35218-35226, 2011.

126. Erez N, Truitt M, Olson P, Hanahan D, Hanahan D. Cancer-Associated Fibroblasts Are Activated in Incipient Neoplasia to Orchestrate Tumor-Promoting Inflammation in an NF-кBDependent Manner. Cancer Cell 17: 135-147, 2010.

127. Esfandiarei M, Luo H, Yanagawa B, Suarez A, Dabiri D, Zhang J, McManus BM. Protein 
kinase B/Akt regulates coxsackievirus B3 replication through a mechanism which is not caspase dependent. J Virol 78: 4289-98, 2004.

128. Fagerholm S, Morrice N, Gahmberg CG, Cohen P. Phosphorylation of the cytoplasmic domain of the integrin CD18 chain by protein kinase C isoforms in leukocytes. J Biol Chem 277: 1728-38, 2002.

129. Fagerholm SC, Hilden TJ, Nurmi SM, Gahmberg CG. Specific integrin $\alpha$ and $\beta$ chain phosphorylations regulate LFA-1 activation through affinity-dependent and -independent mechanisms. J Cell Biol 171: 705-715, 2005.

130. Fairweather D, Stafford KA, Sung YK. Update on coxsackievirus B3 myocarditis. Curr Opin Rheumatol 24: 401-7, 2012.

131. Fan Z, McArdle S, Marki A, Mikulski Z, Gutierrez E, Engelhardt B, Deutsch U, Ginsberg M, Groisman A, Ley K. Neutrophil recruitment limited by high-affinity bent $\beta 2$ integrin binding ligand in cis. Nat Commun 7: 12658, 2016.

132. Fässler R, Meyer M. Consequences of lack of $\beta 1$ integrin gene expression in mice. Genes Dev 9: 1896-908, 1995.

133. Favre B, Fontao L, Koster J, Shafaatian R, Jaunin F, Saurat JH, Sonnenberg A, Borradori $\mathbf{L}$. The hemidesmosomal protein bullous pemphigoid antigen 1 and the integrin $\beta 4$ subunit bind to ERBIN. Molecular cloning of multiple alternative splice variants of ERBIN and analysis of their tissue expression. J Biol Chem 276: 32427-36, 2001.

134. Felding-Habermann B, O'Toole TE, Smith JW, Fransvea E, Ruggeri ZM, Ginsberg MH, Hughes PE, Pampori N, Shattil SJ, Saven A, Mueller BM. Integrin activation controls metastasis in human breast cancer. Proc Natl Acad Sci U S A 98: 1853-8, 2001.

135. Fillingham I, Gingras AR, Papagrigoriou E, Patel B, Emsley J, Critchley DR, Roberts GCK, Barsukov IL. A vinculin binding domain from the talin rod unfolds to form a complex with the vinculin head. Structure 13: 65-74, 2005.

136. Fiore VF, Strane PW, Bryksin A V., White ES, Hagood JS, Barker TH. Conformational coupling of integrin and Thy-1 regulates Fyn priming and fibroblast mechanotransduction. $J$ Cell Biol 211: 173-190, 2015.

137. van der Flier A, Kuikman I, Kramer D, Geerts D, Kreft M, Takafuta T, Shapiro SS, Sonnenberg A. Different splice variants of filamin-B affect myogenesis, subcellular distribution, and determine binding to integrin $\beta$ subunits. J Cell Biol 156: 361-376, 2002.

138. Folkman J, Watson K, Ingber D, Hanahan D. Induction of angiogenesis during the transition from hyperplasia to neoplasia. Nature 339: 58-61, 1989.

139. Follain G, Osmani N, Azevedo AS, Allio G, Mercier L, Karreman MA, Solecki G, Garcia Leòn MJ, Lefebvre O, Fekonja N, Hille C, Chabannes V, Dollé G, Metivet T, Hovsepian F Der, Prudhomme C, Pichot A, Paul N, Carapito R, Bahram S, Ruthensteiner B, Kemmling A, Siemonsen S, Schneider T, Fiehler J, Glatzel M, Winkler F, Schwab Y, Pantel K, Harlepp S, Goetz JG. Hemodynamic Forces Tune the Arrest, Adhesion, and Extravasation of Circulating Tumor Cells. Dev Cell 45: 33-52.e12, 2018.

140. Foster CT, Gualdrini F, Treisman R. Mutual dependence of the MRTF-SRF and YAPTEAD pathways in cancer-associated fibroblasts is indirect and mediated by cytoskeletal 
dynamics. Genes Dev 31: 2361-2375, 2017.

141. De Franceschi N, Arjonen A, Elkhatib N, Denessiouk K, Wrobel AG, Wilson TA, Pouwels J, Montagnac G, Owen DJ, Ivaska J. Selective integrin endocytosis is driven by interactions between the integrin $\alpha$-chain and AP2. Nat Struct Mol Biol 23: 172-179, 2016.

142. De Franceschi N, Hamidi H, Alanko J, Sahgal P, Ivaska J. Integrin traffic - the update. $J$ Cell Sci 128: 839-52, 2015.

143. De Franceschi N, Peuhu E, Parsons M, Rissanen S, Vattulainen I, Salmi M, Ivaska J, Pouwels J. Mutually Exclusive Roles of SHARPIN in Integrin Inactivation and NF- $\kappa B$ Signaling. PLoS One 10: e0143423, 2015.

144. Franco SJ, Rodgers MA, Perrin BJ, Han J, Bennin DA, Critchley DR, Huttenlocher A. Calpain-mediated proteolysis of talin regulates adhesion dynamics [Online]. Nat Cell Biol 6: 977-983, 2004. http://www.nature.com/ncb/journal/v6/n10/suppinfo/ncb1175_S1.html.

145. Freeman TC, Black JL, Bray HG, Dagliyan O, Wu YI, Tripathy A, Dokholyan N V., Leisner TM, Parise L V. Identification of Novel Integrin Binding Partners for Calcium and Integrin Binding Protein 1 (CIB1): Structural and Thermodynamic Basis of CIB1 Promiscuity. Biochemistry 52: 7082-7090, 2013.

146. Friedland JC, Lee MH, Boettiger D. Mechanically activated integrin switch controls $\alpha 5 \beta 1$ function. Science 323: 642-4, 2009.

147. Frisch SM, Francis H. Disruption of epithelial cell-matrix interactions induces apoptosis. $J$ Cell Biol 124: 619-26, 1994.

148. Gahmberg CG, Fagerholm SC, Nurmi SM, Chavakis T, Marchesan S, Grönholm M. Regulation of integrin activity and signalling. Biochim Biophys Acta 1790: 431-44, 2009.

149. Gao J, Huang M, Lai J, Mao K, Sun P, Cao Z, Hu Y, Zhang Y, Schulte ML, Jin C, Wang J, White GC, Xu Z, Ma Y-Q. Kindlin supports platelet integrin $\alpha I I b \beta 3$ activation by interacting with paxillin. J Cell Sci 130: 3764-3775, 2017.

150. García-Alvarez B, de Pereda JM, Calderwood DA, Ulmer TS, Critchley DR, Campbell ID, Ginsberg MH, Liddington RC. Structural Determinants of Integrin Recognition by Talin. Mol Cell 11: 49-58, 2003.

151. Gardel ML, Schneider IC, Aratyn-Schaus Y, Waterman CM. Mechanical integration of actin and adhesion dynamics in cell migration. Annu Rev Cell Dev Biol 26: 315-33, 2010.

152. Garrigues HJ, Rubinchikova YE, Dipersio CM, Rose TM. Integrin $\alpha$ V $\beta 3$ Binds to the RGD motif of glycoprotein B of Kaposi's sarcoma-associated herpesvirus and functions as an RGDdependent entry receptor. J Virol 82: 1570-80, 2008.

153. Gauthier NC, Roca-Cusachs $\mathbf{P}$. Mechanosensing at integrin-mediated cell-matrix adhesions: from molecular to integrated mechanisms. Curr Opin Cell Biol 50: 20-26, 2018.

154. Geiger C, Nagel W, Boehm T, van Kooyk Y, Figdor CG, Kremmer E, Hogg N, Zeitlmann L, Dierks H, Weber KS, Kolanus W. Cytohesin-1 regulates $\beta-2$ integrin-mediated adhesion through both ARF-GEF function and interaction with LFA-1. EMBO J 19: 2525-36, 2000.

155. Georges-Labouesse E, Messaddeq N, Yehia G, Cadalbert L, Dierich A, Le Meur M. Absence of integrin $\alpha 6$ leads to epidermolysis bullosa and neonatal death in mice. Nat Genet 
13: 370-3, 1996.

156. Georgiadou M, Ivaska J. Tensins: Bridging AMP-Activated Protein Kinase with Integrin Activation. Trends Cell Biol 27: 703-711, 2017.

157. Georgiadou M, Lilja J, Jacquemet G, Guzmán C, Rafaeva M, Alibert C, Yan Y, Sahgal P, Lerche M, Manneville J-B, Mäkelä TP, Ivaska J. AMPK negatively regulates tensindependent integrin activity. J Cell Biol 216: 1107-1121, 2017.

158. Giancotti FG, Comoglio PM, Tarone G. A 135,000 molecular weight plasma membrane glycoprotein involved in fibronectin-mediated cell adhesion. Immunofluorescence localization in normal and RSV-transformed fibroblasts. Exp Cell Res 163: 47-62, 1986.

159. Gingras AR, Ziegler WH, Bobkov AA, Joyce MG, Fasci D, Himmel M, Rothemund S, Ritter A, Grossmann JG, Patel B, Bate N, Goult BT, Emsley J, Barsukov IL, Roberts GCK, Liddington RC, Ginsberg MH, Critchley DR. Structural Determinants of Integrin Binding to the Talin Rod. J Biol Chem 284: 8866-8876, 2009.

160. Gingras AR, Ziegler WH, Frank R, Barsukov IL, Roberts GCK, Critchley DR, Emsley J. Mapping and Consensus Sequence Identification for Multiple Vinculin Binding Sites within the Talin Rod. J Biol Chem 280: 37217-37224, 2005.

161. Glenney JR, Zokas L. Novel tyrosine kinase substrates from Rous sarcoma virus-transformed cells are present in the membrane skeleton. J Cell Biol 108: 2401-8, 1989.

162. Le Goff E, Vallentin A, Harmand P-O, Aldrian-Herrada G, Rebière B, Roy C, Benyamin Y, Lebart M-C. Characterization of L-plastin interaction with $\beta$ integrin and its regulation by micro-calpain. Cytoskeleton 67: 286-296, 2010.

163. Goksoy E, Ma Y-Q, Wang X, Kong X, Perera D, Plow EF, Qin J. Structural basis for the autoinhibition of talin in regulating integrin activation. Mol Cell 31: 124-133, 2008.

164. Gontier Y, Taivainen A, Fontao L, Sonnenberg A, van der Flier A, Carpen O, Faulkner G, Borradori L. The Z-disc proteins myotilin and FATZ-1 interact with each other and are connected to the sarcolemma via muscle-specific filamins. J Cell Sci 118: 3739-3749, 2005.

165. Goodman SL, Picard M. Integrins as therapeutic targets. Trends Pharmacol Sci 33: 405-12, 2012.

166. Goodwin K, Ellis SJ, Lostchuck E, Zulueta-Coarasa T, Fernandez-Gonzalez R, Tanentzapf G. Basal Cell-Extracellular Matrix Adhesion Regulates Force Transmission during Tissue Morphogenesis. Dev Cell 39: 611-625, 2016.

167. Gordon FH, Lai CW, Hamilton MI, Allison MC, Srivastava ED, Fouweather MG, Donoghue S, Greenlees C, Subhani J, Amlot PL, Pounder RE. A randomized placebocontrolled trial of a humanized monoclonal antibody to $\alpha 4$ integrin in active Crohn's disease. Gastroenterology 121: 268-74, 2001.

168. Gottlieb A, Krueger JG, Bright R, Ling M, Lebwohl M, Kang S, Feldman S, Spellman M, Wittkowski K, Ochs HD, Jardieu P, Bauer R, White M, Dedrick R, Garovoy M. Effects of administration of a single dose of a humanized monoclonal antibody to CD11a on the immunobiology and clinical activity of psoriasis. J Am Acad Dermatol 42: 428-35, 2000.

169. Goult BT, Yan J, Schwartz MA. Talin as a mechanosensitive signaling hub. J Cell Biol 217: 
3776-3784, 2018.

170. Goult BT, Zacharchenko T, Bate N, Tsang R, Hey F, Gingras AR, Elliott PR, Roberts GCK, Ballestrem C, Critchley DR, Barsukov IL. RIAM and Vinculin Binding to Talin Are Mutually Exclusive and Regulate Adhesion Assembly and Turnover. J Biol Chem 288: 82388249, 2013.

171. Graham KL, Fleming FE, Halasz P, Hewish MJ, Nagesha HS, Holmes IH, Takada Y, Coulson BS. Rotaviruses interact with $\alpha 4 \beta 7$ and $\alpha 4 \beta 1$ integrins by binding the same integrin domains as natural ligands. J Gen Virol 86: 3397-408, 2005.

172. Green HJ, Griffiths AG, Ylänne J, Brown NH. Novel functions for integrin-associated proteins revealed by analysis of myofibril attachment in Drosophila. Elife 7, 2018.

173. Grove J, Marsh M. The cell biology of receptor-mediated virus entry. J Cell Biol 195: 10711082, 2011.

174. Gudzenko T, Franz CM. Studying early stages of fibronectin fibrillogenesis in living cells by atomic force microscopy. Mol Biol Cell 26: 3190-204, 2015.

175. Gupta S, Chit JC-Y, Feng C, Bhunia A, Tan S-M, Bhattacharjya S. An Alternative Phosphorylation Switch in Integrin $\beta 2$ (CD18) Tail for Dok1 Binding. Sci Rep 5: 11630, 2015.

176. Gushiken FC, Patel V, Liu Y, Pradhan S, Bergeron AL, Peng Y, Vijayan KV. Protein Phosphatase 2A Negatively Regulates Integrin $\alpha_{\text {IIb }} \beta_{3}$ Signaling. J Biol Chem 283: $12862-$ $12869,2008$.

177. Haining AWM, Lieberthal TJ, del Río Hernández A. Talin: a mechanosensitive molecule in health and disease. FASEB J 30: 2073-2085, 2016.

178. Haining AWM, Rahikainen R, Cortes E, Lachowski D, Rice A, von Essen M, Hytönen VP, Del Río Hernández A. Mechanotransduction in talin through the interaction of the R8 domain with DLC1. PLoS Biol 16: e2005599, 2018.

179. Han J, Lim CJ, Watanabe N, Soriani A, Ratnikov B, Calderwood DA, PuzonMcLaughlin W, Lafuente EM, Boussiotis VA, Shattil SJ, Ginsberg MH. Reconstructing and Deconstructing Agonist-Induced Activation of Integrin $\alpha$ IIbß3. Curr Biol 16: 1796-1806, 2006.

180. Han J, Liu S, Rose DM, Schlaepfer DD, McDonald H, Ginsberg MH. Phosphorylation of the integrin $\alpha 4$ cytoplasmic domain regulates paxillin binding. J Biol Chem 276: 40903-9, 2001 .

181. Hannigan GE, Leung-Hagesteijn C, Fitz-Gibbon L, Coppolino MG, Radeva G, Filmus J, Bell JC, Dedhar S. Regulation of cell adhesion and anchorage-dependent growth by a new $\beta$ 1-integrin-linked protein kinase. Nature 379: 91-6, 1996.

182. Harburger DS, Bouaouina M, Calderwood DA. Kindlin-1 and -2 Directly Bind the Cterminal Region of B Integrin Cytoplasmic Tails and Exert Integrin-specific Activation Effects. J Biol Chem 284: 11485-11497, 2009.

183. Harrington RA, Kleiman NS, Kottke-Marchant K, Lincoff AM, Tcheng JE, Sigmon KN, Joseph D, Rios G, Trainor K, Rose D. Immediate and reversible platelet inhibition after intravenous administration of a peptide glycoprotein IIb/IIIa inhibitor during percutaneous 
coronary intervention. Am J Cardiol 76: 1222-7, 1995.

184. Harris PC, Torres VE. Polycystic kidney disease. Annu Rev Med 60: 321-37, 2009.

185. Has C, Nyström A, Saeidian AH, Bruckner-Tuderman L, Uitto J. Epidermolysis bullosa: Molecular pathology of connective tissue components in the cutaneous basement membrane zone. Matrix Biol 71-72: 313-329, 2018.

186. Hauck CR, Borisova M, Muenzner P. Exploitation of integrin function by pathogenic microbes. Curr Opin Cell Biol 24: 637-44, 2012.

187. Helsten TL, Bunch TA, Kato H, Yamanouchi J, Choi SH, Jannuzi AL, Féral CC, Ginsberg MH, Brower DL, Shattil SJ. Differences in regulation of Drosophila and vertebrate integrin affinity by talin. Mol Biol Cell 19: 3589-98, 2008.

188. Hermann M-R, Jakobson M, Colo GP, Rognoni E, Jakobson M, Kupatt C, Posern G, Fässler R. Integrins synergise to induce expression of the MRTF-A-SRF target gene ISG15 for promoting cancer cell invasion. J Cell Sci 129: 1391-1403, 2016.

189. Hildreth JE, August JT. The human lymphocyte function-associated (HLFA) antigen and a related macrophage differentiation antigen (HMac-1): functional effects of subunit-specific monoclonal antibodies. J Immunol 134: 3272-80, 1985.

190. Hinz B. The extracellular matrix and transforming growth factor- $\beta 1$ : Tale of a strained relationship. Matrix Biol 47: 54-65, 2015.

191. Hirata E, Girotti MR, Viros A, Hooper S, Spencer-Dene B, Matsuda M, Larkin J, Marais R, Sahai E. Intravital Imaging Reveals How BRAF Inhibition Generates Drug-Tolerant Microenvironments with High Integrin 31/FAK Signaling. Cancer Cell 27: 574-588, 2015.

192. Hodivala-Dilke KM, DiPersio CM, Kreidberg JA, Hynes RO. Novel roles for $\alpha 3 \beta 1$ integrin as a regulator of cytoskeletal assembly and as a trans-dominant inhibitor of integrin receptor function in mouse keratinocytes. J Cell Biol 142: 1357-69, 1998.

193. Hofer U, Syfrig J, Chiquet-Ehrismann R. Identification and characterization of a dimeric chicken fibronectin receptor. Subunit-specific monoclonal antibodies to the putative chicken $\alpha$ $5 \beta 1$ integrin. $J$ Biol Chem 265: 14561-5, 1990.

194. Horton ER, Byron A, Askari JA, Ng DHJ, Millon-Frémillon A, Robertson J, Koper EJ, Paul NR, Warwood S, Knight D, Humphries JD, Humphries MJ. Definition of a consensus integrin adhesome and its dynamics during adhesion complex assembly and disassembly. Nat Cell Biol 17: 1577-1587, 2015.

195. Hoshino D, Branch KM, Weaver AM. Signaling inputs to invadopodia and podosomes. $J$ Cell Sci 126: 2979-89, 2013.

196. Hu J, Niu M, Li X, Lu D, Cui J, Xu W, Li G, Zhan J, Zhang H. FERM domain-containing protein FRMD5 regulates cell motility via binding to integrin $\beta 5$ subunit and ROCK1. FEBS Lett 588: 4348-4356, 2014.

197. Huang DL, Bax NA, Buckley CD, Weis WI, Dunn AR. Vinculin forms a directionally asymmetric catch bond with F-actin. Science 357: 703-706, 2017.

198. Huang XZ, Wu JF, Cass D, Erle DJ, Corry D, Young SG, Farese R V, Sheppard D. Inactivation of the integrin $\beta 6$ subunit gene reveals a role of epithelial integrins in regulating 
inflammation in the lung and skin. J Cell Biol 133: 921-8, 1996.

199. Humphries JD, Chastney MR, Askari JA, Humphries MJ. Signal transduction via integrin adhesion complexes. Curr Opin Cell Biol 56: 14-21, 2019.

200. Humphries JD, Wang P, Streuli C, Geiger B, Humphries MJ, Ballestrem C. Vinculin controls focal adhesion formation by direct interactions with talin and actin. J Cell Biol 179: 1043-57, 2007.

201. Hussein HAM, Walker LR, Abdel-Raouf UM, Desouky SA, Montasser AKM, Akula SM. Beyond RGD: virus interactions with integrins. Arch Virol 160: 2669-81, 2015.

202. Hynes RO. Alteration of cell-surface proteins by viral transformation and by proteolysis. Proc Natl Acad Sci U S A 70: 3170-4, 1973.

203. Hynes RO. Integrins: Bidirectional, Allosteric Signaling Machines. Cell 110: 673-687, 2002.

204. Hynes RO, Destree AT. Relationships between fibronectin (LETS protein) and actin. Cell 15: 875-86, 1978.

205. Hytönen VP, Smith ML, Vogel V. Translating mechanical force into discrete biochemical signal changes: Multimodularity imposes unique properties to mechanotransductive proteins. In: Cellular mechanotransduction : diverse perspectives from molecules to tissues, edited by Mofrad MRK, Kamm RD, Vogel V. Cambridge University Press, 2010, p. 286-338.

206. Hytönen VP, Vogel V. How Force Might Activate Talin's Vinculin Binding Sites: SMD Reveals a Structural Mechanism. PLoS Comput Biol 4: e24, 2008.

207. Hytönen VP, Wehrle-Haller B. Protein conformation as a regulator of cell-matrix adhesion. Phys Chem Chem Phys 16: 6342-57, 2014.

208. Hytönen VP, Wehrle-Haller B. Mechanosensing in cell-matrix adhesions - Converting tension into chemical signals. Exp Cell Res 343: 35-41, 2016.

209. Ingber DE, Folkman J. Mechanochemical switching between growth and differentiation during fibroblast growth factor-stimulated angiogenesis in vitro: role of extracellular matrix. $J$ Cell Biol 109: 317-30, 1989.

210. Isberg RR, Leong JM. Multiple $\beta 1$ chain integrins are receptors for invasin, a protein that promotes bacterial penetration into mammalian cells. Cell 60: 861-71, 1990.

211. Ithychanda SS, Hsu D, Li H, Yan L, Liu D, Das M, Plow EF, Qin J, Qin J. Identification and Characterization of Multiple Similar Ligand-binding Repeats in Filamin. J Biol Chem 284: 35113-35121, 2009.

212. Iwai H, Kim M, Yoshikawa Y, Ashida H, Ogawa M, Fujita Y, Muller D, Kirikae T, Jackson PK, Kotani S, Sasakawa C. A bacterial effector targets Mad2L2, an APC inhibitor, to modulate host cell cycling. Cell 130: 611-23, 2007.

213. Jokinen J, White DJ, Salmela M, Huhtala M, Käpylä J, Sipilä K, Puranen JS, Nissinen L, Kankaanpää P, Marjomäki V, Hyypiä T, Johnson MS, Heino J. Molecular mechanism of $\alpha 2 \beta 1$ integrin interaction with human echovirus 1. EMBO J 29: 196-208, 2010.

214. Jones A V, Kreil S, Zoi K, Waghorn K, Curtis C, Zhang L, Score J, Seear R, Chase AJ, Grand FH, White H, Zoi C, Loukopoulos D, Terpos E, Vervessou E-C, Schultheis B, 
Emig M, Ernst T, Lengfelder E, Hehlmann R, Hochhaus A, Oscier D, Silver RT, Reiter A, Cross NCP. Widespread occurrence of the JAK2 V617F mutation in chronic myeloproliferative disorders. Blood 106: 2162-8, 2005.

215. Kadler KE, Hill A, Canty-Laird EG. Collagen fibrillogenesis: fibronectin, integrins, and minor collagens as organizers and nucleators. Curr Opin Cell Biol 20: 495-501, 2008.

216. Kalluri R, Zeisberg M. Fibroblasts in cancer. Nat Rev Cancer 6: 392-401, 2006.

217. Kanchanawong P, Shtengel G, Pasapera AM, Ramko EB, Davidson MW, Hess HF, Waterman CM. Nanoscale architecture of integrin-based cell adhesions. Nature 468: 580$584,2010$.

218. Kato A, Kawamata N, Tamayose K, Egashira M, Miura R, Fujimura T, Murayama K, Oshimi K. Ancient Ubiquitous Protein 1 Binds to the Conserved Membrane-proximal Sequence of the Cytoplasmic Tail of the Integrin $\alpha$ Subunits That Plays a Crucial Role in the Inside-out Signaling of $\alpha_{\text {Iाb }} \beta_{3}$. J Biol Chem 277: 28934-28941, 2002.

219. Kato A, Oshimi K. Ancient ubiquitous protein 1 and Syk link cytoplasmic tails of the integrin $\boldsymbol{\alpha}_{\text {IIb }} \boldsymbol{\beta}_{3 .}$. Platelets 20: 105-110, 2009.

220. Kendall T, Mukai L, Jannuzi AL, Bunch TA. Identification of integrin $\beta$ subunit mutations that alter affinity for extracellular matrix ligand. J Biol Chem 286: 30981-93, 2011.

221. Kerstein PC, Patel KM, Gomez TM. Calpain-Mediated Proteolysis of Talin and FAK Regulates Adhesion Dynamics Necessary for Axon Guidance. J Neurosci 37: 1568-1580, 2017.

222. Khoshnoodi J, Pedchenko V, Hudson BG. Mammalian collagen IV. Microsc Res Tech 71: 357-70, 2008.

223. Kiema T, Lad Y, Jiang P, Oxley CL, Baldassarre M, Wegener KL, Campbell ID, Ylänne J, Calderwood DA. The molecular basis of filamin binding to integrins and competition with talin. Mol Cell 21: 337-47, 2006.

224. Kim M, Ogawa M, Fujita Y, Yoshikawa Y, Nagai T, Koyama T, Nagai S, Lange A,

Fässler R, Sasakawa C. Bacteria hijack integrin-linked kinase to stabilize focal adhesions and block cell detachment. Nature 459: 578-82, 2009.

225. Kim S-M, Kwon MS, Park CS, Choi K-R, Chun J-S, Ahn J, Song WK. Modulation of Thr Phosphorylation of Integrin $\beta 1$ during Muscle Differentiation. J Biol Chem 279: 7082-7090, 2004.

226. King SL, Kamata T, Cunningham JA, Emsley J, Liddington RC, Takada Y, Bergelson JM. Echovirus 1 interaction with the human very late antigen-2 (integrin $\alpha 2 \beta 1$ ) I domain. Identification of two independent virus contact sites distinct from the metal ion-dependent adhesion site. J Biol Chem 272: 28518-22, 1997.

227. Kirk RI, Sanderson MR, Lerea KM. Threonine phosphorylation of the $\beta 3$ integrin cytoplasmic tail, at a site recognized by PDK1 and Akt/PKB in vitro, regulates Shc binding. $J$ Biol Chem 275: 30901-6, 2000.

228. Klapholz B, Brown NH. Talin - the master of integrin adhesions. J Cell Sci 130: 2435-2446, 2017. 
229. Klapholz B, Herbert SL, Wellmann J, Johnson R, Parsons M, Brown NH. Alternative Mechanisms for Talin to Mediate Integrin Function. Curr Biol 25: 847-857, 2015.

230. Klapproth S, Sperandio M, Pinheiro EM, Prünster M, Soehnlein O, Gertler FB, Fässler R, Moser M. Loss of the Rap1 effector RIAM results in leukocyte adhesion deficiency due to impaired $\beta 2$ integrin function in mice. Blood 126: 2704-12, 2015.

231. Kloeker S, Major MB, Calderwood DA, Ginsberg MH, Jones DA, Beckerle MC. The Kindler Syndrome Protein Is Regulated by Transforming Growth Factor- $\beta$ and Involved in Integrin-mediated Adhesion. J Biol Chem 279: 6824-6833, 2004.

232. Kolanus W, Nagel W, Schiller B, Zeitlmann L, Godar S, Stockinger H, Seed B. A L $\beta 2$ integrin/LFA-1 binding to ICAM-1 induced by cytohesin-1, a cytoplasmic regulatory molecule. Cell 86: 233-42, 1996.

233. Kong F, García AJ, Mould AP, Humphries MJ, Zhu C. Demonstration of catch bonds between an integrin and its ligand. J Cell Biol 185: 1275-84, 2009.

234. Koster J, van Wilpe S, Kuikman I, Litjens SHM, Sonnenberg A. Role of Binding of Plectin to the Integrin $\beta 4$ Subunit in the Assembly of Hemidesmosomes. Mol Biol Cell 15: 1211-1223, 2004.

235. Kreidberg JA, Donovan MJ, Goldstein SL, Rennke H, Shepherd K, Jones RC, Jaenisch R. A $3 \beta 1$ integrin has a crucial role in kidney and lung organogenesis. Development 122: 3537-47, 1996.

236. Kubow KE, Vukmirovic R, Zhe L, Klotzsch E, Smith ML, Gourdon D, Luna S, Vogel V. Mechanical forces regulate the interactions of fibronectin and collagen I in extracellular matrix. Nat Commun 6: 8026, 2015.

237. Kukkurainen S, Määttä JA, Saeger J, Valjakka J, Vogel V, Hytönen VP. The talinintegrin interface under mechanical stress. Mol BioSyst 10: 3217-3228, 2014.

238. Kumar A, Anderson KL, Swift MF, Hanein D, Volkmann N, Schwartz MA. Local Tension on Talin in Focal Adhesions Correlates with F-Actin Alignment at the Nanometer Scale. Biophys J 115: 1569-1579, 2018.

239. Kuo J-C, Han X, Hsiao C-T, Yates III JR, Waterman CM. Analysis of the myosin-IIresponsive focal adhesion proteome reveals a role for $\beta$-Pix in negative regulation of focal adhesion maturation. Nat Cell Biol 13: 383-393, 2011.

240. Lampi MC, Reinhart-King CA. Targeting extracellular matrix stiffness to attenuate disease: From molecular mechanisms to clinical trials. Sci Transl Med 10: eaao0475, 2018.

241. Larkin D, Murphy D, Reilly DF, Cahill M, Sattler E, Harriott P, Cahill DJ, Moran N. ICln, a novel integrin $\alpha \operatorname{Ilb} \beta 3$-associated protein, functionally regulates platelet activation. $J$ Biol Chem 279: 27286-93, 2004.

242. Lau TL, Partridge AW, Ginsberg MH, Ulmer TS. Structure of the integrin $\beta 3$ transmembrane segment in phospholipid bicelles and detergent micelles. Biochemistry 47: 4008-4016, 2008.

243. Lee D-Y, Li Y-SJ, Chang S-F, Zhou J, Ho H-M, Chiu J-J, Chien S. Oscillatory flowinduced proliferation of osteoblast-like cells is mediated by $\alpha v \beta 3$ and $\beta 1$ integrins through 
synergistic interactions of focal adhesion kinase and Shc with phosphatidylinositol 3-kinase and the Akt/mTOR/p70S6K pathway. J Biol Chem 285: 30-42, 2010.

244. Lee H-S, Lim CJ, Puzon-McLaughlin W, Shattil SJ, Ginsberg MH. RIAM activates integrins by linking talin to ras GTPase membrane-targeting sequences. J Biol Chem 284: 5119-27, 2009.

245. Lee JO, Bankston LA, Arnaout MA, Liddington RC. Two conformations of the integrin Adomain (I-domain): a pathway for activation? Structure 3: 1333-40, 1995.

246. Lee K, Boctor S, Barisoni LMC, Gusella GL. Inactivation of integrin- $\beta 1$ prevents the development of polycystic kidney disease after the loss of polycystin-1. J Am Soc Nephrol 26: 888-95, 2015.

247. Lee SE, Kamm RD, Mofrad MRK. Force-induced activation of Talin and its possible role in focal adhesion mechanotransduction. J Biomech 40: 2096-2106, 2007.

248. Lefort CT, Rossaint J, Moser M, Petrich BG, Zarbock A, Monkley SJ, Critchley DR, Ginsberg MH, Fässler R, Ley K. Distinct roles for talin-1 and kindlin-3 in LFA-1 extension and affinity regulation. Blood 119: 4275-4282, 2012.

249. Lemke SB, Weidemann T, Cost A-L, Grashoff C, Schnorrer F. A small proportion of Talin molecules transmit forces to achieve muscle attachment in vivo. bioRxiv (October 17, 2018). doi: $10.1101 / 446336$.

250. Lenter M, Vestweber $\mathbf{D}$. The integrin chains $\beta 1$ and $\alpha 6$ associate with the chaperone calnexin prior to integrin assembly. J Biol Chem 269: 12263-8, 1994.

251. Leong JM, Fournier RS, Isberg RR. Identification of the integrin binding domain of the Yersinia pseudotuberculosis invasin protein. EMBO J 9: 1979-89, 1990.

252. Li G, Du X, Vass WC, Papageorge AG, Lowy DR, Qian X. Full activity of the deleted in liver cancer 1 (DLC1) tumor suppressor depends on an LD-like motif that binds talin and focal adhesion kinase (FAK). Proc Natl Acad Sci 108: 17129-17134, 2011.

253. Li H, Deng Y, Sun K, Yang H, Liu J, Wang M, Zhang Z, Lin J, Wu C, Wei Z, Yu C. Structural basis of kindlin-mediated integrin recognition and activation. Proc Natl Acad Sci U S A 114: 9349-9354, 2017.

254. Li J, Ballif BA, Powelka AM, Dai J, Gygi SP, Hsu VW. Phosphorylation of ACAP1 by Akt Regulates the Stimulation-Dependent Recycling of Integrin $\beta 1$ to Control Cell Migration. Dev Cell 9: 663-673, 2005.

255. Li J, Mayne R, Wu C. A novel muscle-specific $\beta 1$ integrin binding protein (MIBP) that modulates myogenic differentiation. J Cell Biol 147: 1391-8, 1999.

256. Li J, Rao H, Burkin D, Kaufman SJ, Wu C. The muscle integrin binding protein (MIBP) interacts with $\alpha 7 \beta 1$ integrin and regulates cell adhesion and laminin matrix deposition. Dev Biol 261: 209-19, 2003.

257. Li J, Su Y, Xia W, Qin Y, Humphries MJ, Vestweber D, Cabañas C, Lu C, Springer TA. Conformational equilibria and intrinsic affinities define integrin activation. EMBO J 36: 629645, 2017.

258. Liliental J, Chang DD. Rack1, a receptor for activated protein kinase C, interacts with integrin 
$\beta$ subunit. J Biol Chem 273: 2379-83, 1998.

259. Lim S-T, Chen XL, Lim Y, Hanson DA, Vo T-T, Howerton K, Larocque N, Fisher SJ, Schlaepfer DD, Ilic D. Nuclear FAK Promotes Cell Proliferation and Survival through FERMEnhanced p53 Degradation. Mol Cell 29: 9-22, 2008.

260. Lin H-Y, Su Y-F, Hsieh M-T, Lin S, Meng R, London D, Lin C, Tang H-Y, Hwang J, Davis FB, Mousa SA, Davis PJ. Nuclear monomeric integrin $\alpha$ v in cancer cells is a coactivator regulated by thyroid hormone. FASEB J 27: 3209-16, 2013.

261. Lindert S, Silvestry M, Mullen T-M, Nemerow GR, Stewart PL. Cryo-electron microscopy structure of an adenovirus-integrin complex indicates conformational changes in both penton base and integrin. $J$ Virol 83: 11491-501, 2009.

262. Ling K, Doughman RL, Firestone AJ, Bunce MW, Anderson RA. Type I gamma phosphatidylinositol phosphate kinase targets and regulates focal adhesions. Nature 420: 8993, 2002.

263. La Linn M, Eble JA, Lübken C, Slade RW, Heino J, Davies J, Suhrbier A. An arthritogenic $\alpha$ virus uses the $\alpha 1 \beta 1$ integrin collagen receptor. Virology 336: 229-39, 2005.

264. Liu J, Das M, Yang J, Ithychanda SS, Yakubenko VP, Plow EF, Qin J. Structural mechanism of integrin inactivation by filamin. Nat Struct Mol Biol 22: 383-9, 2015.

265. Liu J, Wang Y, Goh WI, Goh H, Baird MA, Ruehland S, Teo S, Bate N, Critchley DR, Davidson MW, Kanchanawong P. Talin determines the nanoscale architecture of focal adhesions. Proc Natl Acad Sci U S A 112: E4864-73, 2015.

266. Liu S, Ginsberg MH. Paxillin binding to a conserved sequence motif in the $\alpha 4$ integrin cytoplasmic domain. J Biol Chem 275: 22736-42, 2000.

267. Liu W, Draheim KM, Zhang R, Calderwood DA, Boggon TJ. Mechanism for KRIT1 release of ICAP1-mediated suppression of integrin activation. Mol Cell 49: 719-29, 2013.

268. Liu Y, Guan L, Zhan J, Lu D, Wan J, Zhang H. FERM domain-containing unconventional myosin VIIA interacts with integrin $\beta 5$ subunit and regulates $\alpha v \beta 5$-mediated cell adhesion and migration. FEBS Lett 588: 2859-2866, 2014.

269. Lobert VH, Brech A, Pedersen NM, Wesche J, Oppelt A, Malerød L, Stenmark H. Ubiquitination of $\alpha 5 \beta 1$ integrin controls fibroblast migration through lysosomal degradation of fibronectin-integrin complexes. Dev Cell 19: 148-59, 2010.

270. Lock JG, Jones MC, Askari JA, Gong X, Oddone A, Olofsson H, Göransson S, Lakadamyali M, Humphries MJ, Strömblad S. Reticular adhesions are a distinct class of cell-matrix adhesions that mediate attachment during mitosis. Nat Cell Biol 20: 1290-1302, 2018.

271. Lu Z, Mathew S, Chen J, Hadziselimovic A, Palamuttam R, Hudson BG, Fässler R, Pozzi A, Sanders CR, Zent R. Implications of the differing roles of the $\beta 1$ and $\beta 3$ transmembrane and cytoplasmic domains for integrin function. Elife 5: e18633, 2016.

272. Luo B-H, Springer TA, Takagi J. Stabilizing the open conformation of the integrin headpiece with a glycan wedge increases affinity for ligand. Proc Natl Acad Sci U S A 100: 2403-8, 2003. 
273. Macpherson I, Montagnier L. Agar suspension culture for the selective assay of cells transformed by polyoma virus. Virology 23: 291-4, 1964.

274. Mahabeleshwar GH, Feng W, Reddy K, Plow EF, Byzova T V. Mechanisms of integrinvascular endothelial growth factor receptor cross-activation in angiogenesis. Circ Res 101: 570-80, 2007.

275. Mai A, Veltel S, Pellinen T, Padzik A, Coffey E, Marjomäki V, Ivaska J. Competitive binding of Rab21 and p120RasGAP to integrins regulates receptor traffic and migration. $J$ Cell Biol 194: 291-306, 2011.

276. Major EO. Progressive multifocal leukoencephalopathy in patients on immunomodulatory therapies. Аnпu Rev Med 61: 35-47, 2010.

277. Malinin NL, Zhang L, Choi J, Ciocea A, Razorenova O, Ma Y-Q, Podrez EA, Tosi M, Lennon DP, Caplan AI, Shurin SB, Plow EF, Byzova T V. A point mutation in KINDLIN3 ablates activation of three integrin subfamilies in humans. Nat Med 15: 313-8, 2009.

278. Mana G, Clapero F, Panieri E, Panero V, Böttcher RT, Tseng H-Y, Saltarin F, Astanina E, Wolanska KI, Morgan MR, Humphries MJ, Santoro MM, Serini G, Valdembri D. PPFIA1 drives active $\alpha 5 \beta 1$ integrin recycling and controls fibronectin fibrillogenesis and vascular morphogenesis. Nat Commun 7: 13546, 2016.

279. Marjoram RJ, Li Z, He L, Tollefsen DM, Kunicki TJ, Dickeson SK, Santoro SA, Zutter MM. $\alpha 2 \beta 1$ Integrin, GPVI Receptor, and Common FcR $\gamma$ Chain on Mouse Platelets Mediate Distinct Responses to Collagen in Models of Thrombosis. PLoS One 9: e114035, 2014.

280. Marsh M, Helenius A. Virus Entry: Open Sesame. Cell 124: 729-740, 2006.

281. Marshall BT, Long M, Piper JW, Yago T, McEver RP, Zhu C. Direct observation of catch bonds involving cell-adhesion molecules. Nature 423: 190-3, 2003.

282. Mathew S, Chen X, Pozzi A, Zent R. Integrins in renal development. Pediatr Nephrol 27: 891-900, 2012.

283. McCarty JH, Cook AA, Hynes RO. An interaction between $\{\alpha\} \vee\{\beta\} 8$ integrin and Band 4.1B via a highly conserved region of the Band 4.1 C-terminal domain. Proc Natl Acad Sci US A 102: 13479-83, 2005.

284. McCleverty CJ, Lin DC, Liddington RC. Structure of the PTB domain of tensin1 and a model for its recruitment to fibrillar adhesions. Protein Sci 16: 1223-9, 2007.

285. Meng Z, Qiu Y, Lin KC, Kumar A, Placone JK, Fang C, Wang K-C, Lu S, Pan M, Hong AW, Moroishi T, Luo M, Plouffe SW, Diao Y, Ye Z, Park HW, Wang X, Yu F-X, Chien S, Wang C-Y, Ren B, Engler AJ, Guan K-L. RAP2 mediates mechanoresponses of the Hippo pathway. Nature 560: 655-660, 2018.

286. Meredith JE, Fazeli B, Schwartz MA. The extracellular matrix as a cell survival factor. Mol Biol Cell 4: 953-61, 1993.

287. Midwood KS, Williams LV, Schwarzbauer JE. Tissue repair and the dynamics of the extracellular matrix. Int J Biochem Cell Biol 36: 1031-7, 2004.

288. Miller CG, Pozzi A, Zent R, Schwarzbauer JE. Effects of high glucose on integrin activity and fibronectin matrix assembly by mesangial cells. Mol Biol Cell 25: 2342-50, 2014. 
289. Miyazaki N, Iwasaki K, Takagi J. A systematic survey of conformational states in $\beta 1$ and $\beta 4$ integrins using negative-stain electron microscopy. J Cell Sci 131: jcs216754, 2018.

290. Moes M, Rodius S, Coleman SJ, Monkley SJ, Goormaghtigh E, Tremuth L, Kox C, van der Holst PPG, Critchley DR, Kieffer N. The Integrin Binding Site 2 (IBS2) in the Talin Rod Domain Is Essential for Linking Integrin ß Subunits to the Cytoskeleton. J Biol Chem 282: 17280-17288, 2007.

291. Moore SW, Roca-Cusachs P, Sheetz MP. Stretchy proteins on stretchy substrates: the important elements of integrin-mediated rigidity sensing. Dev Cell 19: 194-206, 2010.

292. Moore TI, Aaron J, Chew T-L, Springer TA. Measuring Integrin Conformational Change on the Cell Surface with Super-Resolution Microscopy. Cell Rep 22: 1903-1912, 2018.

293. Moreno-Layseca P, Icha J, Hamidi H, Ivaska J. Integrin trafficking in cells and tissues. Nat Cell Biol 21: 122-132, 2019.

294. Moser M, Bauer M, Schmid S, Ruppert R, Schmidt S, Sixt M, Wang H-V, Sperandio M, Fassler R. Kindlin-3 is required for $[\beta] 2$ integrin-mediated leukocyte adhesion to endothelial cells. Nat Med 15: 300-305, 2009.

295. Moser M, Nieswandt B, Ussar S, Pozgajova M, Fässler R. Kindlin-3 is essential for integrin activation and platelet aggregation. Nat Med 14: 325-330, 2008.

296. Mosesson MW, Umfleet RA. The cold-insoluble globulin of human plasma. I. Purification, primary characterization, and relationship to fibrinogen and other cold-insoluble fraction components. J Biol Chem 245: 5728-36, 1970.

297. Muenzner P, Rohde M, Kneitz S, Hauck CR. CEACAM engagement by human pathogens enhances cell adhesion and counteracts bacteria-induced detachment of epithelial cells. J Cell Biol 170: 825-36, 2005.

298. Müller B, Zerwes HG, Tangemann K, Peter J, Engel J. Two-step binding mechanism of fibrinogen to $\alpha$ IIb $\beta 3$ integrin reconstituted into planar lipid bilayers. J Biol Chem 268: 68008, 1993.

299. Munger JS, Huang X, Kawakatsu H, Griffiths MJ, Dalton SL, Wu J, Pittet JF, Kaminski N, Garat C, Matthay MA, Rifkin DB, Sheppard D. The integrin $\alpha \vee \beta 6$ binds and activates latent TGF $\beta$ 1: a mechanism for regulating pulmonary inflammation and fibrosis. Cell 96: 319-28, 1999.

300. Murphy DA, Courtneidge SA. The "ins" and "outs" of podosomes and invadopodia: characteristics, formation and function. Nat Rev Mol Cell Biol 12: 413-426, 2011.

301. Mykuliak V V, Haining AWM, von Essen M, Del Río Hernández A, Hytönen VP. Mechanical unfolding reveals stable 3-helix intermediates in talin and $\alpha$-catenin. PLoS Comput Biol 14: e1006126, 2018.

302. Nagae M, Re S, Mihara E, Nogi T, Sugita Y, Takagi J. Crystal structure of $\alpha 5 \beta 1$ integrin ectodomain: atomic details of the fibronectin receptor. J Cell Biol 197: 131-40, 2012.

303. Naik UP, Patel PM, Parise L V. Identification of a novel calcium-binding protein that interacts with the integrin $\alpha$ IIb cytoplasmic domain. J Biol Chem 272: 4651-4, 1997.

304. Nandrot EF, Kim Y, Brodie SE, Huang X, Sheppard D, Finnemann SC. Loss of 
synchronized retinal phagocytosis and age-related blindness in mice lacking $\alpha v \beta 5$ integrin. $J$ Exp Med 200: 1539-45, 2004.

305. Nardone G, Oliver-De La Cruz J, Vrbsky J, Martini C, Pribyl J, Skládal P, Pešl M, Caluori G, Pagliari S, Martino F, Maceckova Z, Hajduch M, Sanz-Garcia A, Pugno NM, Stokin GB, Forte G. YAP regulates cell mechanics by controlling focal adhesion assembly. Nat Commun 8: 15321, 2017.

306. Nejjari M, Couvelard A, Mosnier JF, Moreau A, Feldmann G, Degott C, Marcellin P, Scoazec JY. Integrin up-regulation in chronic liver disease: relationship with inflammation and fibrosis in chronic hepatitis C. J Pathol 195: 473-81, 2001.

307. van der Neut R, Krimpenfort P, Calafat J, Niessen CM, Sonnenberg A. Epithelial detachment due to absence of hemidesmosomes in integrin $\beta 4$ null mice. Nat Genet 13: 366-9, 1996.

308. Nevo J, Mai A, Tuomi S, Pellinen T, Pentikäinen OT, Heikkilä P, Lundin J, Joensuu H, Bono P, Ivaska J. Mammary-derived growth inhibitor (MDGI) interacts with integrin $\alpha$ subunits and suppresses integrin activity and invasion. Oncogene 29: 6452-63, 2010.

309. Nieswandt B. Glycoprotein VI but not $\alpha 2 \beta 1$ integrin is essential for platelet interaction with collagen. EMBO J 20: 2120-2130, 2001.

310. Niewoehner DE, Kleinerman J, Rice DB. Pathologic changes in the peripheral airways of young cigarette smokers. N Engl J Med 291: 755-8, 1974.

311. Nishimura T, Kaibuchi K. Numb Controls Integrin Endocytosis for Directional Cell Migration with aPKC and PAR-3. Dev Cell 13: 15-28, 2007.

312. Nissinen L, Koivunen J, Käpylä J, Salmela M, Nieminen J, Jokinen J, Sipilä K, Pihlavisto M, Pentikäinen OT, Marjamäki A, Heino J. Novel $\alpha 2 \beta 1$ integrin inhibitors reveal that integrin binding to collagen under shear stress conditions does not require receptor preactivation. J Biol Chem 287: 44694-702, 2012.

313. Nordenfelt P, Elliott HL, Springer TA. Coordinated integrin activation by actin-dependent force during T-cell migration. 7: 13119, 2016.

314. Obergfell A, Eto K, Mocsai A, Buensuceso C, Moores SL, Brugge JS, Lowell CA, Shattil SJ. Coordinate interactions of Csk, Src, and Syk kinases with $\alpha \mathrm{IIb} \beta 3$ initiate integrin signaling to the cytoskeleton. J Cell Biol 157: 265-275, 2002.

315. Oksala N, Pärssinen J, Seppälä I, Klopp N, Illig T, Laaksonen R, Levula M, Raitoharju E, Kholova I, Sioris T, Kähönen M, Lehtimäki T, Hytönen VP. Kindlin 3 (FERMT3) is associated with unstable atherosclerotic plaques, anti-inflammatory type II macrophages and upregulation of $\beta-2$ integrins in all major arterial beds. Atherosclerosis 242: 145-54, 2015.

316. Orimo A, Weinberg RA. Stromal Fibroblasts in Cancer: A Novel Tumor-Promoting Cell Type. Cell Cycle 5: 1597-1601, 2006.

317. Ortiz Franyuti D, Mitsi M, Vogel V. Mechanical Stretching of Fibronectin Fibers Upregulates Binding of Interleukin-7. Nano Lett 18: 15-25, 2018.

318. Otey CA, Pavalko FM, Burridge K. An interaction between $\alpha$-actinin and the $\beta 1$ integrin subunit in vitro. J Cell Biol 111: 721-9, 1990. 
319. Overbeek SA, Braber S, Henricks PAJ, Kleinjan M, Kamp VM, Georgiou NA, Garssen J, Kraneveld AD, Folkerts G. Cigarette smoke induces $\beta 2$-integrin-dependent neutrophil migration across human endothelium. Respir Res 12: 75, 2011.

320. Oxley CL, Anthis NJ, Lowe ED, Vakonakis I, Campbell ID, Wegener L. An Integrin Phosphorylation Switch. J Biol Chem 283: 5420-5426, 2008.

321. Ozawa A, Sato Y, Imabayashi T, Uemura T, Takagi J, Sekiguchi K. Molecular Basis of the Ligand Binding Specificity of avß8 Integrin. J Biol Chem 291: 11551-65, 2016.

322. Pan D. The Hippo Signaling Pathway in Development and Cancer. Dev Cell 19: 491-505, 2010.

323. Panciera T, Azzolin L, Cordenonsi M, Piccolo S. Mechanobiology of YAP and TAZ in physiology and disease. Nat Rev Mol Cell Biol 18: 758-770, 2017.

324. Pankov R, Cukierman E, Katz BZ, Matsumoto K, Lin DC, Lin S, Hahn C, Yamada KM. Integrin dynamics and matrix assembly: tensin-dependent translocation of $\alpha(5) \beta(1)$ integrins promotes early fibronectin fibrillogenesis. J Cell Biol 148: 1075-90, 2000.

325. De Paoli F, Staels B, Chinetti-Gbaguidi G. Macrophage phenotypes and their modulation in atherosclerosis. Circ J 78: 1775-81, 2014.

326. Park H, Valencia-Gallardo C, Sharff A, Tran Van Nhieu G, Izard T. Novel vinculin binding site of the IpaA invasin of Shigella. J Biol Chem 286: 23214-21, 2011.

327. Parsons JT, Horwitz AR, Schwartz MA. Cell adhesion: integrating cytoskeletal dynamics and cellular tension. Nat Rev Mol Cell Biol 11: 633-643, 2010.

328. Paul NR, Jacquemet G, Caswell PT. Endocytic Trafficking of Integrins in Cell Migration. Curr Biol 25: R1092-105, 2015.

329. Pavalko FM, Otey CA, Simon KO, Burridge K. A-actinin: a direct link between actin and integrins. Biochem Soc Trans 19: 1065-9, 1991.

330. Peacock SJ, Day NP, Thomas MG, Berendt AR, Foster TJ. Clinical isolates of Staphylococcus aureus exhibit diversity in fnb genes and adhesion to human fibronectin. $J$ Infect 41: 23-31, 2000.

331. Pellinen T, Arjonen A, Vuoriluoto K, Kallio K, Fransen JAM, Ivaska J. Small GTPase Rab21 regulates cell adhesion and controls endosomal traffic of $\beta 1$-integrins. J Cell Biol 173: 767-780, 2006.

332. de Pereda JM, Lillo MP, Sonnenberg A. Structural basis of the interaction between integrin $\alpha 6 \beta 4$ and plectin at the hemidesmosomes. EMBO J 28: 1180-90, 2009.

333. Pfaff M, Jurdic P. Podosomes in osteoclast-like cells: structural analysis and cooperative roles of paxillin, proline-rich tyrosine kinase 2 (Pyk2) and integrin $\alpha \mathrm{V} \beta 3$. J Cell Sci 114: 2775-86, 2001 .

334. Pierschbacher MD, Ruoslahti E. Cell attachment activity of fibronectin can be duplicated by small synthetic fragments of the molecule. Nature 309: 30-3, [date unknown].

335. Pinon P, Pärssinen J, Vazquez P, Bachmann M, Rahikainen R, Jacquier M-C, Azizi L, Määttä JA, Bastmeyer M, Hytönen VP, Wehrle-Haller B. Talin-bound NPLY motif recruits 
integrin-signaling adapters to regulate cell spreading and mechanosensing. J Cell Biol 205: 265-281, 2014.

336. Plançon S, Morel-Kopp MC, Schaffner-Reckinger E, Chen P, Kieffer N. Green fluorescent protein (GFP) tagged to the cytoplasmic tail of $\alpha$ IIb or $\beta 3$ allows the expression of a fully functional integrin $\alpha \operatorname{Ilb}(\beta 3)$ : effect of $\beta 3 \mathrm{GFP}$ on $\alpha \operatorname{IIb}(\beta 3)$ ligand binding. Biochem J 357: 529 36, 2001.

337. Podolnikova NP, O'Toole TE, Haas TA, Lam SC-T, Fox JEB, Ugarova TP. Adhesioninduced unclasping of cytoplasmic tails of integrin $\alpha(\mathrm{IIb}) \beta 3$. Biochemistry 48: 617-29, 2009.

338. Poincloux R, Lizarraga F, Chavrier P. Matrix invasion by tumour cells: a focus on MT1MMP trafficking to invadopodia. J Cell Sci 122: 3015-3024, 2009.

339. Polman CH, O’Connor PW, Havrdova E, Hutchinson M, Kappos L, Miller DH, Phillips JT, Lublin FD, Giovannoni G, Wajgt A, Toal M, Lynn F, Panzara MA, Sandrock AW, AFFIRM Investigators. A randomized, placebo-controlled trial of natalizumab for relapsing multiple sclerosis. N Engl J Med 354: 899-910, 2006.

340. Popov Y, Patsenker E, Stickel F, Zaks J, Bhaskar KR, Niedobitek G, Kolb A, Friess H, Schuppan D. Integrin $\alpha v \beta 6$ is a marker of the progression of biliary and portal liver fibrosis and a novel target for antifibrotic therapies. J Hepatol 48: 453-64, 2008.

341. Pöschl E, Schlötzer-Schrehardt U, Brachvogel B, Saito K, Ninomiya Y, Mayer U. Collagen IV is essential for basement membrane stability but dispensable for initiation of its assembly during early development. Development 131: 1619-28, 2004.

342. Potten CS. Stem cells in gastrointestinal epithelium: numbers, characteristics and death. Philos Trans R Soc B Biol Sci 353: 821-830, 1998.

343. Pouwels J, De Franceschi N, Rantakari P, Auvinen K, Karikoski M, Mattila E, Potter C, Sundberg JP, Hogg N, Gahmberg CG, Salmi M, Ivaska J. SHARPIN regulates uropod detachment in migrating lymphocytes. Cell Rep 5: 619-28, 2013.

344. Pouwels J, Nevo J, Pellinen T, Ylanne J, Ivaska J. Negative regulators of integrin activity. J Cell Sci 125: 3271-3280, 2012.

345. Praekelt U, Kopp PM, Rehm K, Linder S, Bate N, Patel B, Debrand E, Manso AM, Ross RS, Conti F, Zhang M-Z, Harris RC, Zent R, Critchley DR, Monkley SJ. New isoformspecific monoclonal antibodies reveal different sub-cellular localisations for talin1 and talin2. Eur J Cell Biol 91: 180-91, 2012.

346. Prager GW, Féral CC, Kim C, Han J, Ginsberg MH. CD98hc (SLC3A2) interaction with the integrin $\beta$ subunit cytoplasmic domain mediates adhesive signaling. J Biol Chem 282: 24477-84, 2007.

347. Puklin-Faucher E, Gao M, Schulten K, Vogel V. How the headpiece hinge angle is opened: new insights into the dynamics of integrin activation. J Cell Biol 175: 349-360, 2006.

348. Pulido D, Hussain S-A, Hohenester E. Crystal Structure of the Heterotrimeric IntegrinBinding Region of Laminin-111. Structure 25: 530-535, 2017.

349. Pytela R, Pierschbacher MD, Ruoslahti E. Identification and isolation of a $140 \mathrm{kd}$ cell surface glycoprotein with properties expected of a fibronectin receptor. Cell 40: 191-198, 
1985.

350. Pytela R, Pierschbacher MD, Ruoslahti E. A 125/115-kDa cell surface receptor specific for vitronectin interacts with the arginine-glycine-aspartic acid adhesion sequence derived from fibronectin. Proc Natl Acad Sci U S A 82: 5766-70, 1985.

351. Qasim H, Karim ZA, Silva-Espinoza JC, Khasawneh FT, Rivera JO, Ellis CC, Bauer SL, Almeida IC, Alshbool FZ. Short-Term E-Cigarette Exposure Increases the Risk of Thrombogenesis and Enhances Platelet Function in Mice. J Am Heart Assoc 7, 2018.

352. Raghavan S, Bauer C, Mundschau G, Li Q, Fuchs E. Conditional ablation of $\beta 1$ integrin in skin. Severe defects in epidermal proliferation, basement membrane formation, and hair follicle invagination. J Cell Biol 150: 1149-60, 2000.

353. Rahikainen R, von Essen M, Schaefer M, Qi L, Azizi L, Kelly C, Ihalainen TO, WehrleHaller B, Bastmeyer M, Huang C, Hytönen VP. Mechanical stability of talin rod controls cell migration and substrate sensing. Sci Rep 7: 3571, 2017.

354. Rahikainen R, Öhman T, Turkki P, Varjosalo M, Hytönen VP. Talin-mediated force transmission and talin rod domain unfolding independently regulate adhesion signaling. J Cell Sci 132: jcs226514, 2019.

355. Rainero E, Howe JD, Caswell PT, Jamieson NB, Anderson K, Critchley DR, Machesky L, Norman JC. Ligand-Occupied Integrin Internalization Links Nutrient Signaling to Invasive Migration. Cell Rep 10: 398-413, 2015.

356. Rakshit S, Zhang Y, Manibog K, Shafraz O, Sivasankar S. Ideal, catch, and slip bonds in cadherin adhesion. Proc Natl Acad Sci U S A 109: 18815-20, 2012.

357. Ramsay AG, Keppler MD, Jazayeri M, Thomas GJ, Parsons M, Violette S, Weinreb P, Hart IR, Marshall JF. HS1-Associated Protein X-1 Regulates Carcinoma Cell Migration and Invasion via Clathrin-Mediated Endocytosis of Integrin $\alpha_{v} \beta_{6}$. Cancer Res 67: 5275-5284, 2007.

358. Rankin S, Isberg RR, Leong JM. The integrin-binding domain of invasin is sufficient to allow bacterial entry into mammalian cells. Infect Immun 60: 3909-12, 1992.

359. Rantala JK, Pouwels J, Pellinen T, Veltel S, Laasola P, Mattila E, Potter CS, Duffy T, Sundberg JP, Kallioniemi O, Askari JA, Humphries MJ, Parsons M, Salmi M, Ivaska J. SHARPIN is an endogenous inhibitor of $\beta 1$-integrin activation. Nat Cell Biol 13: 1315-1324, 2011.

360. Reddy KB, Gascard P, Price MG, Negrescu E V, Fox JE. Identification of an interaction between the $\mathrm{m}$-band protein skelemin and $\beta$-integrin subunits. Colocalization of a skeleminlike protein with $\beta 1$ - and $\beta 3$-integrins in non-muscle cells. J Biol Chem 273: 35039-47, 1998.

361. Reed NI, Jo H, Chen C, Tsujino K, Arnold TD, DeGrado WF, Sheppard D. The av $\beta 1$ integrin plays a critical in vivo role in tissue fibrosis. Sci Transl Med 7: 288ra79, 2015.

362. Reynolds AR, Hart IR, Watson AR, Welti JC, Silva RG, Robinson SD, Da Violante G, Gourlaouen M, Salih M, Jones MC, Jones DT, Saunders G, Kostourou V, Perron-Sierra F, Norman JC, Tucker GC, Hodivala-Dilke KM. Stimulation of tumor growth and angiogenesis by low concentrations of RGD-mimetic integrin inhibitors. Nat Med 15: 392-400, 2009. 
363. Rietzler M, Bittner M, Kolanus W, Schuster A, Holzmann B. The human WD repeat protein WAIT-1 specifically interacts with the cytoplasmic tails of $\beta 7$-integrins. J Biol Chem 273: 27459-66, 1998.

364. Ringer P, Colo G, Fässler R, Grashoff C. Sensing the mechano-chemical properties of the extracellular matrix. Matrix Biol 64: 6-16, 2017.

365. del Rio A, Perez-Jimenez R, Liu R, Roca-Cusachs P, Fernandez JM, Sheetz MP. Stretching Single Talin Rod Molecules Activates Vinculin Binding. Science (80- ) 323: 638 LP-641, 2009.

366. Riveline D, Zamir E, Balaban NQ, Schwarz US, Ishizaki T, Narumiya S, Kam Z, Geiger B, Bershadsky AD. Focal contacts as mechanosensors: externally applied local mechanical force induces growth of focal contacts by an mDia1-dependent and ROCK-independent mechanism. J Cell Biol 153: 1175-86, 2001.

367. Roberts MS, Woods AJ, Shaw PE, Norman JC. ERK1 associates with $\alpha(v) \beta 3$ integrin and regulates cell spreading on vitronectin. J Biol Chem 278: 1975-85, 2003.

368. Robertson IB, Rifkin DB. Regulation of the Bioavailability of TGF- $\beta$ and TGF- $\beta$-Related Proteins. Cold Spring Harb Perspect Biol 8: a021907, 2016.

369. Roca-Cusachs P, Gauthier NC, del Rio A, Sheetz MP. Clustering of $\alpha(5) \beta(1)$ integrins determines adhesion strength whereas $\alpha(v) \beta(3)$ and talin enable mechanotransduction. Proc Natl Acad Sci U S A 106: 16245-16250, 2009.

370. Rodius S, Chaloin O, Moes M, Schaffner-Reckinger E, Landrieu I, Lippens G, Lin M, Zhang J, Kieffer $\mathbf{N}$. The Talin Rod IBS2 a-Helix Interacts with the B3 Integrin Cytoplasmic Tail Membrane-proximal Helix by Establishing Charge Complementary Salt Bridges. J Biol Chem 283: 24212-24223, 2008.

371. Rognoni E, Ruppert R, Fässler R. The kindlin family: functions, signaling properties and implications for human disease. J Cell Sci 129: 17-27, 2016.

372. Rosario M, Dirks NL, Milch C, Parikh A, Bargfrede M, Wyant T, Fedyk E, Fox I. A Review of the Clinical Pharmacokinetics, Pharmacodynamics, and Immunogenicity of Vedolizumab. Clin Pharmacokinet 56: 1287-1301, 2017.

373. Rossier O, Octeau V, Sibarita J-B, Leduc C, Tessier B, Nair D, Gatterdam V, Destaing O, Albigès-Rizo C, Tampé R, Cognet L, Choquet D, Lounis B, Giannone G. Integrins $\beta 1$ and $\beta 3$ exhibit distinct dynamic nanoscale organizations inside focal adhesions. Nat Cell Biol 14: 1057-1067, 2012.

374. Ruoslahti E. Fibronectin and its integrin receptors in cancer. Adv Cancer Res 76: 1-20, 1999.

375. Ruoslahti E, Vaheri A, Kuusela P, Linder E. Fibroblast surface antigen: a new serum protein. Biochim Biophys Acta 322: 352-8, 1973.

376. Sabra H, Brunner M, Mandati V, Wehrle-Haller B, Lallemand D, Ribba A-S, Chevalier G, Guardiola P, Block MR, Bouvard D. $\beta 1$ integrin-dependent Rac/group I PAK signaling mediates YAP activation of Yes-associated protein 1 (YAP1) via NF2/merlin. J Biol Chem 292: 19179-19197, 2017.

377. Sachs N, Claessen N, Aten J, Kreft M, Teske GJD, Koeman A, Zuurbier CJ, Janssen H, 
Sonnenberg A. Blood pressure influences end-stage renal disease of Cd151 knockout mice. $J$ Clin Invest 122: 348-58, 2012.

378. Sachs N, Kreft M, van den Bergh Weerman MA, Beynon AJ, Peters TA, Weening JJ, Sonnenberg A. Kidney failure in mice lacking the tetraspanin CD151. J Cell Biol 175: 33-9, 2006.

379. Salmela M, Jokinen J, Tiitta S, Rappu P, Cheng RH, Heino J. Integrin $\alpha 2 \beta 1$ in nonactivated conformation can induce focal adhesion kinase signaling. Sci Rep 7: 3414, 2017.

380. Saltel F, Mortier E, Hytönen VP, Jacquier M-C, Zimmermann P, Vogel V, Liu W, Wehrle-Haller B. New PI(4,5)P2- and membrane proximal integrin-binding motifs in the talin head control B3-integrin clustering. J Cell Biol 187: 715-731, 2009.

381. Sampath R, Gallagher PJ, Pavalko FM. Cytoskeletal interactions with the leukocyte integrin $\beta 2$ cytoplasmic tail. Activation-dependent regulation of associations with talin and $\alpha$-actinin. $J$ Biol Chem 273: 33588-94, 1998.

382. Samson T, Smyth N, Janetzky S, Wendler O, Müller JM, Schüle R, von der Mark H, von der Mark K, Wixler V. The LIM-only Proteins FHL2 and FHL3 Interact with $\alpha$ - and $\beta$ Subunits of the Muscle $\alpha_{7} \beta_{1}$ Integrin Receptor. J Biol Chem 279: 28641-28652, 2004.

383. Sanchez-Madrid F, Simon P, Thompson S, Springer TA. Mapping of antigenic and functional epitopes on the $\alpha$ - and $\beta$-subunits of two related mouse glycoproteins involved in cell interactions, LFA-1 and Mac-1. J Exp Med 158: 586-602, 1983.

384. Sawada Y, Sheetz MP. Force transduction by Triton cytoskeletons. J Cell Biol 156: 609-615, 2002.

385. Sawada Y, Tamada M, Dubin-Thaler BJ, Cherniavskaya O, Sakai R, Tanaka S, Sheetz MP. Force sensing by mechanical extension of the Src family kinase substrate p130Cas. Cell 127: 1015-26, 2006.

386. Saxena M, Changede R, Hone J, Wolfenson H, Sheetz MP. Force-Induced Calpain Cleavage of Talin Is Critical for Growth, Adhesion Development, and Rigidity Sensing. Nano Lett 17: 7242-7251, 2017.

387. Schiller HB, Friedel CC, Boulegue C, Fässler R. Quantitative proteomics of the integrin adhesome show a myosin II-dependent recruitment of LIM domain proteins. EMBO Rep 12: 259-66, 2011.

388. Schiller HB, Hermann M-R, Polleux J, Vignaud T, Zanivan S, Friedel CC, Sun Z, Raducanu A, Gottschalk K-E, Théry M, Mann M, Fässler R. $\beta 1$ - and $\alpha$ v-class integrins cooperate to regulate myosin II during rigidity sensing of fibronectin-based microenvironments. Nat Cell Biol 15: 625-636, 2013.

389. Schuppan D, Ruehl M, Somasundaram R, Hahn EG. Matrix as a modulator of hepatic fibrogenesis. Semin Liver Dis 21: 351-72, 2001.

390. Schwarzbauer JE, DeSimone DW. Fibronectins, Their Fibrillogenesis, and In Vivo Functions. Cold Spring Harb Perspect Biol 3: a005041-a005041, 2011.

391. Sen M, Yuki K, Springer TA. An internal ligand-bound, metastable state of a leukocyte integrin, $\alpha$ Xß2. J Cell Biol 203: 629-42, 2013. 
392. Senetar MA, Moncman CL, McCann RO. Talin2 is induced during striated muscle differentiation and is targeted to stable adhesion complexes in mature muscle. Cell Motil Cytoskeleton 64: 157-173, 2007.

393. Shakeel S, Seitsonen JJT, Kajander T, Laurinmäki P, Hyypiä T, Susi P, Butcher SJ. Structural and functional analysis of coxsackievirus A9 integrin $\alpha v \beta 6$ binding and uncoating. $J$ Virol 87: 3943-51, 2013.

394. Shattil SJ, Hoxie JA, Cunningham M, Brass LF. Changes in the platelet membrane glycoprotein IIb.IIIa complex during platelet activation. J Biol Chem 260: 11107-14, 1985.

395. Shattil SJ, O'Toole T, Eigenthaler M, Thon V, Williams M, Babior BM, Ginsberg MH. B 3-endonexin, a novel polypeptide that interacts specifically with the cytoplasmic tail of the integrin $\beta 3$ subunit. J Cell Biol 131: 807-16, 1995.

396. Sheppard D. In vivo functions of integrins: lessons from null mutations in mice. Matrix Biol 19: 203-9, 2000.

397. Shi F, Sottile J. Caveolin-1-dependent 1 integrin endocytosis is a critical regulator of fibronectin turnover. J Cell Sci 121: 2360-2371, 2008.

398. Shi F, Sottile J. MT1-MMP regulates the turnover and endocytosis of extracellular matrix fibronectin. J Cell Sci 124: 4039-4050, 2011.

399. Shiu J-Y, Aires L, Lin Z, Vogel V. Nanopillar force measurements reveal actin-cap-mediated YAP mechanotransduction. Nat Cell Biol 20: 262-271, 2018.

400. Shock DD, Naik UP, Brittain JE, Alahari SK, Sondek J, Parise L V. Calcium-dependent properties of CIB binding to the integrin $\alpha$ IIb cytoplasmic domain and translocation to the platelet cytoskeleton. Biochem J 342 Pt 3: 729-35, 1999.

401. Shyer AE, Rodrigues AR, Schroeder GG, Kassianidou E, Kumar S, Harland RM. Emergent cellular self-organization and mechanosensation initiate follicle pattern in the avian skin. Science (80- ) 357: 811-815, 2017.

402. Siegel DH, Ashton GHS, Penagos HG, Lee J V., Feiler HS, Wilhelmsen KC, South AP, Smith FJD, Prescott AR, Wessagowit V, Oyama N, Akiyama M, Al Aboud D, Al Aboud K, Al Githami A, Al Hawsawi K, Al Ismaily A, Al-Suwaid R, Atherton DJ, Caputo R, Fine J-D, Frieden IJ, Fuchs E, Haber RM, Harada T, Kitajima Y, Mallory SB, Ogawa H, Sahin S, Shimizu H, Suga Y, Tadini G, Tsuchiya K, Wiebe CB, Wojnarowska F, Zaghloul AB, Hamada T, Mallipeddi R, Eady RAJ, McLean WHI, McGrath JA, Epstein EH. Loss of Kindlin-1, a Human Homolog of the Caenorhabditis elegans Actin-Extracellular-Matrix Linker Protein UNC-112, Causes Kindler Syndrome. Am J Hum Genet 73: 174-187, 2003.

403. Simon DI, Xu H, Ortlepp S, Rogers C, Rao NK. 7E3 monoclonal antibody directed against the platelet glycoprotein IIb/IIIa cross-reacts with the leukocyte integrin Mac-1 and blocks adhesion to fibrinogen and ICAM-1. Arterioscler Thromb Vasc Biol 17: 528-35, 1997.

404. Simpson MA, Bradley WD, Harburger D, Parsons M, Calderwood DA, Koleske AJ. Direct Interactions with the Integrin $\beta 1$ Cytoplasmic Tail Activate the Abl2/Arg Kinase. J Biol Chem 290: 8360-8372, 2015.

405. Sinha B, Francois P, Que YA, Hussain M, Heilmann C, Moreillon P, Lew D, Krause KH, Peters G, Herrmann M. Heterologously expressed Staphylococcus aureus fibronectin-binding 
proteins are sufficient for invasion of host cells. Infect Immun 68: 6871-8, 2000.

406. Smith CW, Marlin SD, Rothlein R, Toman C, Anderson DC. Cooperative interactions of LFA-1 and Mac-1 with intercellular adhesion molecule-1 in facilitating adherence and transendothelial migration of human neutrophils in vitro. J Clin Invest 83: 2008-17, 1989.

407. Soler D, Chapman T, Yang L-L, Wyant T, Egan R, Fedyk ER. The Binding Specificity and Selective Antagonism of Vedolizumab, an Anti- 47 Integrin Therapeutic Antibody in Development for Inflammatory Bowel Diseases. J Pharmacol Exp Ther 330: 864-875, 2009.

408. Song G, Yang Y, Liu J-H, Casasnovas JM, Shimaoka M, Springer TA, Wang J-H. An atomic resolution view of ICAM recognition in a complex between the binding domains of ICAM-3 and integrin $\alpha$ L $\beta 2$. Proc Natl Acad Sci U S A 102: 3366-71, 2005.

409. Springer TA, Zhu J, Xiao T. Structural basis for distinctive recognition of fibrinogen gammaC peptide by the platelet integrin $\alpha$ IIbß3. J Cell Biol 182: 791-800, 2008.

410. Stehbens SJ, Paszek M, Pemble H, Ettinger A, Gierke S, Wittmann T. CLASPs link focaladhesion-associated microtubule capture to localized exocytosis and adhesion site turnover. Nat Cell Biol 16: 561-73, 2014.

411. Steinberg F, Heesom KJ, Bass MD, Cullen PJ. SNX17 protects integrins from degradation by sorting between lysosomal and recycling pathways. J Cell Biol 197: 219-30, 2012.

412. Stephens LE, Sutherland AE, Klimanskaya I V, Andrieux A, Meneses J, Pedersen RA, Damsky CH. Deletion of $\beta 1$ integrins in mice results in inner cell mass failure and periimplantation lethality. Genes Dev 9: 1883-95, 1995.

413. Stewart PL, Nemerow GR. Cell integrins: commonly used receptors for diverse viral pathogens. Trends Microbiol 15: 500-507, 2007.

414. Stritt S, Wolf K, Lorenz V, Vogtle T, Gupta S, Bosl MR, Nieswandt B. Rap1-GTPinteracting adaptor molecule (RIAM) is dispensable for platelet integrin activation and function in mice. Blood 125: 219-222, 2015.

415. Stupp R, Hegi ME, Gorlia T, Erridge SC, Perry J, Hong Y-K, Aldape KD, Lhermitte B, Pietsch T, Grujicic D, Steinbach JP, Wick W, Tarnawski R, Nam D-H, Hau P, Weyerbrock A, Taphoorn MJB, Shen C-C, Rao N, Thurzo L, Herrlinger U, Gupta T, Kortmann R-D, Adamska K, McBain C, Brandes AA, Tonn JC, Schnell O, Wiegel T, Kim C-Y, Nabors LB, Reardon DA, van den Bent MJ, Hicking C, Markivskyy A, Picard M, Weller M, European Organisation for Research and Treatment of Cancer (EORTC), Canadian Brain Tumor Consortium, CENTRIC study team. Cilengitide combined with standard treatment for patients with newly diagnosed glioblastoma with methylated MGMT promoter (CENTRIC EORTC 26071-22072 study): a multicentre, randomised, open-label, phase 3 trial. Lancet Oncol 15: 1100-8, 2014.

416. Su W, Wynne J, Pinheiro EM, Strazza M, Mor A, Montenont E, Berger J, Paul DS, Bergmeier W, Gertler FB, Philips MR. Rap1 and its effector RIAM are required for lymphocyte trafficking. Blood 126: 2695-703, 2015.

417. Su Y, Xia W, Li J, Walz T, Humphries MJ, Vestweber D, Cabañas C, Lu C, Springer TA. Relating conformation to function in integrin a5ß1. Proc Natl Acad Sci 113: E3872-E3881, 2016. 
418. Sun L, Xu X, Chen Y, Zhou Y, Tan R, Qiu H, Jin L, Zhang W, Fan R, Hong W, Wang T. Rab34 regulates adhesion, migration, and invasion of breast cancer cells. Oncogene 37: 3698$3714,2018$.

419. Sun Z, Guo SS, Fässler R. Integrin-mediated mechanotransduction. J Cell Biol 215: 445-456, 2016.

420. Sun Z, Tseng H-Y, Tan S, Senger F, Kurzawa L, Dedden D, Mizuno N, Wasik AA, Thery M, Dunn AR, Fassler R. Kank2 activates talin, reduces force transduction across integrins and induces central adhesion formation. Nat Cell Biol 18: 941-953, 2016.

421. Svensson L, Howarth K, McDowall A, Patzak I, Evans R, Ussar S, Moser M, Metin A, Fried M, Tomlinson I, Hogg N. Leukocyte adhesion deficiency-III is caused by mutations in KINDLIN3 affecting integrin activation. Nat Med 15: 306-12, 2009.

422. Swaminathan V, Kalappurakkal JM, Mehta SB, Nordenfelt P, Moore TI, Koga N, Baker DA, Oldenbourg R, Tani T, Mayor S, Springer TA, Waterman CM. Actin retrograde flow actively aligns and orients ligand-engaged integrins in focal adhesions. Proc Natl Acad Sci U S A 114: 10648-10653, 2017.

423. Syfrig J, Mann K, Paulsson M. An abundant chick gizzard integrin is the avian $\alpha 1 \beta 1$ integrin heterodimer and functions as a divalent cation-dependent collagen IV receptor. Exp Cell Res 194: 165-73, 1991.

424. Tabone-Eglinger S, Calderin-Sollet Z, Pinon P, Aebischer N, Wehrle-Haller M, Jacquier M-C, Boettiger D, Wehrle-Haller B. Niche anchorage and signaling through membranebound Kit-ligand/c-kit receptor are kinase independent and imatinib insensitive. FASEB J 28: 4441-56, 2014.

425. Tadokoro S, Shattil SJ, Eto K, Tai V, Liddington RC, Pereda JM de, Ginsberg MH, Calderwood DA. Talin Binding to Integrin $\beta$ Tails: A Final Common Step in Integrin Activation. Science (80- ) 302: 103-106, 2003.

426. Taichman DB, Cybulsky MI, Djaffar I, Longenecker BM, Teixidó J, Rice GE, Aruffo A, Bevilacqua MP. Tumor cell surface $\alpha 4 \beta 1$ integrin mediates adhesion to vascular endothelium: demonstration of an interaction with the N-terminal domains of INCAM110/VCAM-1. Cell Regul 2: 347-55, 1991.

427. Takagi J, Petre BM, Walz T, Springer TA. Global Conformational Rearrangements in Integrin Extracellular Domains in Outside-In and Inside-Out Signaling. Cell 110: 599-611, 2002.

428. Takala H, Nurminen E, Nurmi SM, Aatonen M, Strandin T, Takatalo M, Kiema T, Gahmberg CG, Ylänne J, Fagerholm SC. B2 integrin phosphorylation on Thr758 acts as a molecular switch to regulate 14-3-3 and filamin binding. Blood 112: 1853-62, 2008.

429. Takizawa M, Arimori T, Taniguchi Y, Kitago Y, Yamashita E, Takagi J, Sekiguchi K. Mechanistic basis for the recognition of laminin-511 by $\alpha 6 \beta 1$ integrin. Sci Adv 3: e1701497, 2017.

430. Tam SH, Sassoli PM, Jordan RE, Nakada MT. Abciximab (ReoPro, chimeric 7E3 Fab) demonstrates equivalent affinity and functional blockade of glycoprotein IIb/IIIa and $\alpha(\mathrm{v}) \beta 3$ integrins. Circulation 98: 1085-91, 1998. 
431. Tanentzapf G, Devenport D, Godt D, Brown NH. Integrin-dependent anchoring of a stemcell niche. Nat Cell Biol 9: 1413-8, 2007.

432. Tang K, Cai Y, Joshi S, Tovar E, Tucker SC, Maddipati KR, Crissman JD, Repaskey WT, Honn K V. Convergence of eicosanoid and integrin biology: 12-lipoxygenase seeks a partner. Mol Cancer 14: 111, 2015.

433. Tang P, Cao C, Xu M, Zhang L. Cytoskeletal protein radixin activates integrin $\alpha_{\mathrm{M}} \beta_{2}$ by binding to its cytoplasmic tail. FEBS Lett 581: 1103-1108, 2007.

434. Tani TT, Mercurio AM. PDZ Interaction Sites in Integrin $\alpha$ Subunits. J Biol Chem 276: 36535-36542, 2001.

435. Tcheng JE, Harrington RA, Kottke-Marchant K, Kleiman NS, Ellis SG, Kereiakes DJ, Mick MJ, Navetta FI, Smith JE, Worley SJ. Multicenter, randomized, double-blind, placebo-controlled trial of the platelet integrin glycoprotein IIb/IIIa blocker Integrelin in elective coronary intervention. IMPACT Investigators. Circulation 91: 2151-7, 1995.

436. Tefferi A, Vardiman JW. Classification and diagnosis of myeloproliferative neoplasms: the 2008 World Health Organization criteria and point-of-care diagnostic algorithms. Leukemia 22: $14-22,2008$.

437. Theodosiou M, Widmaier M, Böttcher T. R, Rognoni E, Veelders M, Bharadwaj M, Lambacher A, Austen K, Müller J. D, Zent R, Fässler R. Kindlin-2 cooperates with talin to activate integrins and induces cell spreading by directly binding paxillin. Elife 5: e10130, 2016.

438. Thompson AJ, Pillai EK, Dimov IB, Foster SK, Holt CE, Franze K. Rapid changes in tissue mechanics regulate cell behaviour in the developing embryonic brain. Elife 8, 2019.

439. Tomiyama Y, Brojer E, Ruggeri ZM, Shattil SJ, Smiltneck J, Gorski J, Kumar A, Kieber-Emmons T, Kunicki TJ. A molecular model of RGD ligands. Antibody D gene segments that direct specificity for the integrin $\alpha$ IIb $\beta$ 3. J Biol Chem 267: 18085-92, 1992.

440. Torres VE, Harris PC, Pirson Y. Autosomal dominant polycystic kidney disease. Lancet 369: 1287-1301, 2007.

441. Tseng H-Y, Samarelli A V, Kammerer P, Scholze S, Ziegler T, Immler R, Zent R, Sperandio M, Sanders CR, Fässler R, Böttcher RT. LCP1 preferentially binds clasped aMß2 integrin and attenuates leukocyte adhesion under flow. J Cell Sci 131: jcs218214, 2018.

442. Tseng H-Y, Thorausch N, Ziegler T, Meves A, Fässler R, Böttcher RT. Sorting Nexin 31 Binds Multiple $\beta$ Integrin Cytoplasmic Domains and Regulates $\beta 1$ Integrin Surface Levels and Stability. J Mol Biol 426: 3180-3194, 2014.

443. Tu Y, Huang Y, Zhang Y, Hua Y, Wu C. A new focal adhesion protein that interacts with integrin-linked kinase and regulates cell adhesion and spreading. J Cell Biol 153: 585-98, 2001.

444. Ussar S, Moser M, Widmaier M, Rognoni E, Harrer C, Genzel-Boroviczeny O, Fässler R. Loss of Kindlin-1 Causes Skin Atrophy and Lethal Neonatal Intestinal Epithelial Dysfunction. PLoS Genet 4: e1000289, 2008.

445. Valdembri D, Caswell PT, Anderson KI, Schwarz JP, König I, Astanina E, Caccavari F, Norman JC, Humphries MJ, Bussolino F, Serini G. Neuropilin-1/GIPC1 Signaling 
Regulates $\alpha 5 \beta 1$ Integrin Traffic and Function in Endothelial Cells. PLoS Biol 7: e1000025, 2009.

446. Vaynberg J, Fukuda K, Lu F, Bialkowska K, Chen Y, Plow EF, Qin J. Non-catalytic signaling by pseudokinase ILK for regulating cell adhesion. Nat Commun 9: 4465, 2018.

447. Vijayan KV, Liu Y, Li T-T, Bray PF. Protein phosphatase 1 associates with the integrin $\alpha \mathrm{IIb}$ subunit and regulates signaling. J Biol Chem 279: 33039-42, 2004.

448. Vines CM, Potter JW, Xu Y, Geahlen RL, Costello PS, Tybulewicz VL, Lowell CA, Chang PW, Gresham HD, Willman CL. Inhibition of $\beta 2$ integrin receptor and Syk kinase signaling in monocytes by the Src family kinase Fgr. Immunity 15: 507-19, 2001.

449. Vitorino P, Yeung S, Crow A, Bakke J, Smyczek T, West K, McNamara E, EasthamAnderson J, Gould S, Harris SF, Ndubaku C, Ye W. MAP4K4 regulates integrin-FERM binding to control endothelial cell motility. Nature 519: 425-430, 2015.

450. Vogel V. Mechanotransduction involving multimodular proteins: converting force into biochemical signals. Annu Rev Biophys Biomol Struct 35: 459-488, 2006.

451. Wan X, Kim SY, Guenther LM, Mendoza A, Briggs J, Yeung C, Currier D, Zhang H, Mackall C, Li W-J, Tuan RS, Deyrup AT, Khanna C, Helman L. B4 integrin promotes osteosarcoma metastasis and interacts with ezrin. Oncogene 28: 3401-3411, 2009.

452. Wang S, Wu C, Zhang Y, Zhong Q, Sun H, Cao W, Ge G, Li G, Zhang XF, Chen J. Integrin $\alpha 4 \beta 7$ switches its ligand specificity via distinct conformer-specific activation. $J$ Cell Biol 217: 2799-2812, 2018.

453. Warren MS, Bradley WD, Gourley SL, Lin Y-C, Simpson MA, Reichardt LF, Greer CA, Taylor JR, Koleske AJ. Integrin $\beta 1$ Signals through Arg to Regulate Postnatal Dendritic Arborization, Synapse Density, and Behavior. J Neurosci 32: 2824-2834, 2012.

454. Watanabe N, Bodin L, Pandey M, Krause M, Coughlin S, Boussiotis VA, Ginsberg MH, Shattil SJ. Mechanisms and consequences of agonist-induced talin recruitment to platelet

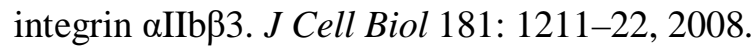

455. Watarai M, Funato S, Sasakawa C. Interaction of Ipa proteins of Shigella flexneri with $\alpha 5 \beta 1$ integrin promotes entry of the bacteria into mammalian cells. J Exp Med 183: 991-9, 1996.

456. Wazawa T, Arai Y, Kawahara Y, Takauchi H, Washio T, Nagai T. Highly biocompatible super-resolution fluorescence imaging using the fast photoswitching fluorescent protein Kohinoor and SPoD-ExPAN with Lp-regularized image reconstruction. Reprod Syst Sex Disord 67: 89-98, 2018.

457. Wegener KL, Partridge AW, Han J, Pickford AR, Liddington RC, Ginsberg MH, Campbell ID. Structural Basis of Integrin Activation by Talin. Cell 128: 171-182, 2007.

458. Wehrle-Haller B. Structure and function of focal adhesions. Curr Opin Cell Biol 24: 116-24, 2012.

459. Wehrle-Haller B. Assembly and disassembly of cell matrix adhesions. Curr Opin Cell Biol 24: 569-81, 2012.

460. Weinert BT, Narita T, Satpathy S, Srinivasan B, Hansen BK, Schölz C, Hamilton WB, Zucconi BE, Wang WW, Liu WR, Brickman JM, Kesicki EA, Lai A, Bromberg KD, Cole 
PA, Choudhary C. Time-Resolved Analysis Reveals Rapid Dynamics and Broad Scope of the CBP/p300 Acetylome. Cell 174: 231-244.e12, 2018.

461. Wells RG. Tissue mechanics and fibrosis. Biochim Biophys Acta - Mol Basis Dis 1832: 884890, 2013.

462. Werther WA, Gonzalez TN, O'Connor SJ, McCabe S, Chan B, Hotaling T, Champe M, Fox JA, Jardieu PM, Berman PW, Presta LG. Humanization of an anti-lymphocyte function-associated antigen (LFA)-1 monoclonal antibody and reengineering of the humanized antibody for binding to rhesus LFA-1. J Immunol 157: 4986-95, 1996.

463. Whittaker CA, Hynes RO. Distribution and Evolution of von Willebrand/Integrin A Domains: Widely Dispersed Domains with Roles in Cell Adhesion and Elsewhere. Mol Biol Cell 13: 3369-3387, 2002.

464. Wickham TJ, Mathias P, Cheresh DA, Nemerow GR. Integrins $\alpha \vee \beta 3$ and $\alpha \vee \beta 5$ promote adenovirus internalization but not virus attachment. Cell 73: 309-19, 1993.

465. Wixler V, Geerts D, Laplantine E, Westhoff D, Smyth N, Aumailley M, Sonnenberg A, Paulsson M. The LIM-only Protein DRAL/FHL2 Binds to the Cytoplasmic Domain of Several $\alpha$ and $\beta$ Integrin Chains and Is Recruited to Adhesion Complexes. J Biol Chem 275: 33669 33678, 2000.

466. Wixler V, Laplantine E, Geerts D, Sonnenberg A, Petersohn D, Eckes B, Paulsson M, Aumailley M. Identification of novel interaction partners for the conserved membrane proximal region of $\alpha$-integrin cytoplasmic domains. FEBS Lett 445: 351-5, 1999.

467. Woods AJ, White DP, Caswell PT, Norman JC. PKD1/PKC $\mu$ promotes $\alpha v \beta 3$ integrin recycling and delivery to nascent focal adhesions. EMBO J 23: 2531-2543, 2004.

468. Wynn T. Cellular and molecular mechanisms of fibrosis. J Pathol 214: 199-210, 2008.

469. Xiao R, Xi X-D, Chen Z, Chen S-J, Meng G. Structural framework of c-Src activation by integrin $\beta 3$. Blood 121: 700-6, 2013.

470. Xiao T, Takagi J, Coller BS, Wang J-H, Springer TA. Structural basis for allostery in integrins and binding to fibrinogen-mimetic therapeutics. Nature 432: 59-67, 2004.

471. Xiong J-P, Stehle T, Diefenbach B, Zhang R, Dunker R, Scott DL, Joachimiak A, Goodman SL, Arnaout MA. Crystal Structure of the Extracellular Segment of Integrin $\alpha$ V $\beta 3$. Science (80- ) 294: 339-345, 2001.

472. Xiong J-P, Stehle T, Zhang R, Joachimiak A, Frech M, Goodman SL, Arnaout MA. Crystal Structure of the Extracellular Segment of Integrin $\alpha \mathrm{V} \beta 3$ in Complex with an Arg-GlyAsp Ligand. Science (80- ) 296: 151-155, 2002.

473. Yamada KM, Pankov R, Cukierman E. Dimensions and dynamics in integrin function. Brazilian J Med Biol Res = Rev Bras Pesqui medicas e Biol 36: 959-66, 2003.

474. Yamada KM, Weston JA. Isolation of a major cell surface glycoprotein from fibroblasts. Proc Natl Acad Sci U S A 71: 3492-6, 1974.

475. Yamodo IH, Blystone SD. Calcium Integrin Binding Protein Associates with Integrins $\alpha$ V $\beta 3$ and $\alpha$ IIb $\beta 3$ Independent of $\beta 3$ Activation Motifs. CellBio 1: 30-37, 2012. 
476. Yan SR, Huang M, Berton G. Signaling by adhesion in human neutrophils: activation of the p72syk tyrosine kinase and formation of protein complexes containing p72syk and Src family kinases in neutrophils spreading over fibrinogen. J Immunol 158: 1902-10, 1997.

477. Yang J, Zhu L, Zhang H, Hirbawi J, Fukuda K, Dwivedi P, Liu J, Byzova T, Plow EF, Wu J, Qin J. Conformational activation of talin by RIAM triggers integrin-mediated cell adhesion. Nat Commun 5: 5880, 2014.

478. Yang X, Dutta U, Shaw LM. SHP2 Mediates the Localized Activation of Fyn Downstream of the $\alpha 6 \beta 4$ Integrin To Promote Carcinoma Invasion. Mol Cell Biol 30: 5306-5317, 2010.

479. Yang X, Kovalenko O V., Tang W, Claas C, Stipp CS, Hemler ME. Palmitoylation supports assembly and function of integrin-tetraspanin complexes. J Cell Biol 167: 1231-1240, 2004.

480. Yang X, Kovalenko O V, Tang W, Claas C, Stipp CS, Hemler ME. Palmitoylation supports assembly and function of integrin-tetraspanin complexes. J Cell Biol 167: 1231-40, 2004.

481. Yang Z, Mu Z, Dabovic B, Jurukovski V, Yu D, Sung J, Xiong X, Munger JS. Absence of integrin-mediated TGF $\beta 1$ activation in vivo recapitulates the phenotype of TGF $\beta 1$-null mice. $J$ Cell Biol 176: 787-93, 2007.

482. Yao M, Goult BT, Chen H, Cong P, Sheetz MP, Yan J. Mechanical activation of vinculin binding to talin locks talin in an unfolded conformation [Online]. Sci Rep 4: 4610, 2014. http://dx.doi.org/10.1038/srep04610.

483. Yazlovitskaya EM, Viquez OM, Tu T, De Arcangelis A, Georges-Labouesse E, Sonnenberg A, Pozzi A, Zent R. The laminin binding $\alpha 3$ and $\alpha 6$ integrins cooperate to promote epithelial cell adhesion and growth. Matrix Biol 77: 101-116, 2019.

484. Ye F, Hu G, Taylor D, Ratnikov B, Bobkov AA, McLean MA, Sligar SG, Taylor KA, Ginsberg MH. Recreation of the terminal events in physiological integrin activation. $J$ Cell Biol 188: 157-173, 2010.

485. Ye F, Kim C, Ginsberg MH. Reconstruction of integrin activation. Blood 119: 26-33, 2012.

486. Ye F, Petrich BG, Anekal P, Lefort CT, Kasirer-Friede A, Shattil SJ, Ruppert R, Moser M, Fässler R, Ginsberg MH. The Mechanism of Kindlin-Mediated Activation of Integrin aIIbß3. Curr Biol 23: 2288-2295, 2013.

487. Yoshida K, Park A-M, Ozaki S, Munakata H. Interaction of calcium- and integrin-binding protein 1 with integrin $\alpha 11$ and its possible involvement in pulmonary fibrosis. Adv Biol Chem 04: 59-66, 2014.

488. Yu C, Rafiq NBM, Cao F, Zhou Y, Krishnasamy A, Biswas KH, Ravasio A, Chen Z, Wang Y-H, Kawauchi K, Jones GE, Sheetz MP. Integrin- $\beta 3$ clusters recruit clathrinmediated endocytic machinery in the absence of traction force. Nat Commun 6: 8672, 2015.

489. Yu Y, Zhu J, Mi L-Z, Walz T, Sun H, Chen J, Springer TA. Structural specializations of $\alpha(4) \beta(7)$, an integrin that mediates rolling adhesion. J Cell Biol 196: 131-46, 2012.

490. Yuan W, Leisner TM, McFadden AW, Wang Z, Larson MK, Clark S, BoudignonProudhon C, Lam SC-T, Parise L V. CIB1 is an endogenous inhibitor of agonist-induced integrin $\alpha$ IIb $\beta 3$ activation. J Cell Biol 172: 169-175, 2006. 
491. Zacharchenko T, Qian X, Goult BT, Jethwa D, Almeida TB, Ballestrem C, Critchley DR, Lowy DR, Barsukov IL. LD Motif Recognition by Talin: Structure of the Talin-DLC1 Complex. Structure 24: 1130-41, 2016.

492. Zaidel-Bar R, Itzkovitz S, Ma'ayan A, Iyengar R, Geiger B. Functional atlas of the integrin adhesome. Nat Cell Biol 9: 858-867, 2007.

493. Zamir E, Geiger B. Molecular complexity and dynamics of cell-matrix adhesions. J Cell Sci 114: 3583-90, 2001.

494. Zent R, Fenczik CA, Calderwood DA, Liu S, Dellos M, Ginsberg MH. Class- and splice variant-specific association of CD98 with integrin $\beta$ cytoplasmic domains. J Biol Chem 275: 5059-64, 2000.

495. Zhang C, Liu J, Jiang X, Haydar N, Zhang C, Shan H, Zhu J. Modulation of integrin activation and signaling by $\alpha 1 / \alpha 1$ '-helix unbending at the junction. J Cell Sci 126: 5735-47, 2013.

496. Z Zhang H, Berg JS, Li Z, Wang Y, Lång P, Sousa AD, Bhaskar A, Cheney RE, Strömblad S. Myosin-X provides a motor-based link between integrins and the cytoskeleton. Nat Cell Biol 6: 523-531, 2004.

497. Zhang H, Li Z, Viklund E-K, Strömblad S. P21-activated kinase 4 interacts with integrin $\alpha$ V $\beta 5$ and regulates $\alpha$ v $\beta$ 5-mediated cell migration. J Cell Biol 158: 1287-97, 2002.

498. Zhang XA, Hemler ME. Interaction of the integrin $\beta 1$ cytoplasmic domain with ICAP-1 protein. J Biol Chem 274: 11-9, 1999.

499. Zhao B, Li L, Wang L, Wang C-Y, Yu J, Guan K-L. Cell detachment activates the Hippo pathway via cytoskeleton reorganization to induce anoikis. Genes Dev 26: 54-68, 2012.

500. Zhu J, Luo B-H, Xiao T, Zhang C, Nishida N, Springer TA. Structure of a complete integrin ectodomain in a physiologic resting state and activation and deactivation by applied forces. Mol Cell 32: 849-61, 2008.

501. Zhu J, Zhu J, Springer TA. Complete integrin headpiece opening in eight steps. J Cell Biol 201: 1053-1068, 2013.

502. Zhu L, Yang J, Bromberger T, Holly A, Lu F, Liu H, Sun K, Klapproth S, Hirbawi J, Byzova T V., Plow EF, Moser M, Qin J. Structure of Rap1b bound to talin reveals a pathway for triggering integrin activation. Nat Commun 8: 1744, 2017.

503. Zuidema A, Wang W, Kreft M, Te Molder L, Hoekman L, Bleijerveld OB, Nahidiazar L, Janssen H, Sonnenberg A. Mechanisms of integrin $\alpha \mathrm{V} \beta 5$ clustering in flat clathrin lattices. $J$ Cell Sci 131: jcs221317, 2018. 

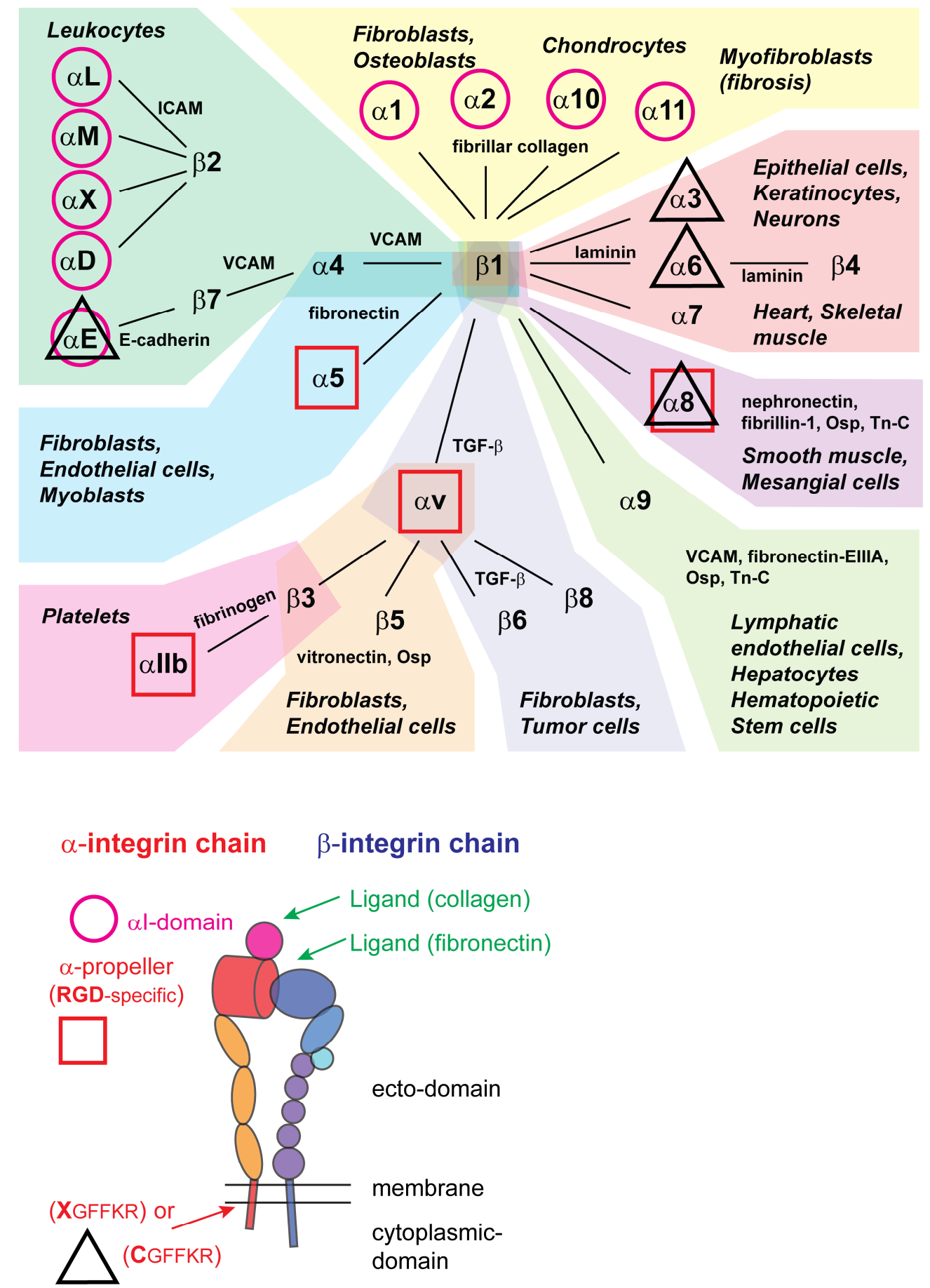

Figure 1 Integrins, their ligands, and cellular distribution.

As reviewed by Hynes (129), integrins are organized in 24 different heterodimers, indicated by the link between $\alpha$ - and $\beta$-subunits in the figure. Integrins can be classified by structural features, their ligands, and their tissue and cellular expression. Based on these criteria, we grouped integrins into nine classes indicated by the color of their background. Typical cell types expressing the respective integrins are mentioned, as well as common ligands for these integrins. We also highlighted integrins with an $\alpha$-I domain (I; purple circle), those binding to RGD ligands (RGD; red square), and those with changes to the conserved GFFKR sequence in the membrane-proximal part of the $\alpha$-subunit (black triangle indicates integrins with sequence 
deviating from CGFFKR). The integrin cartoon in the lower part of the figure gives an overview about the integrin structure and is reused in the following figures. It also indicates the location of the $\alpha \mathrm{I}$ domain and the GFFKR sequence in the respective integrins. Please note that $\alpha \mathrm{I}$ domain integrins bind ligands (e.g. collagen) via this I-domain, while other integrins bind ligands (e.g. fibronectin) in binding pockets formed by both $\alpha$ - and $\beta$-subunits. 


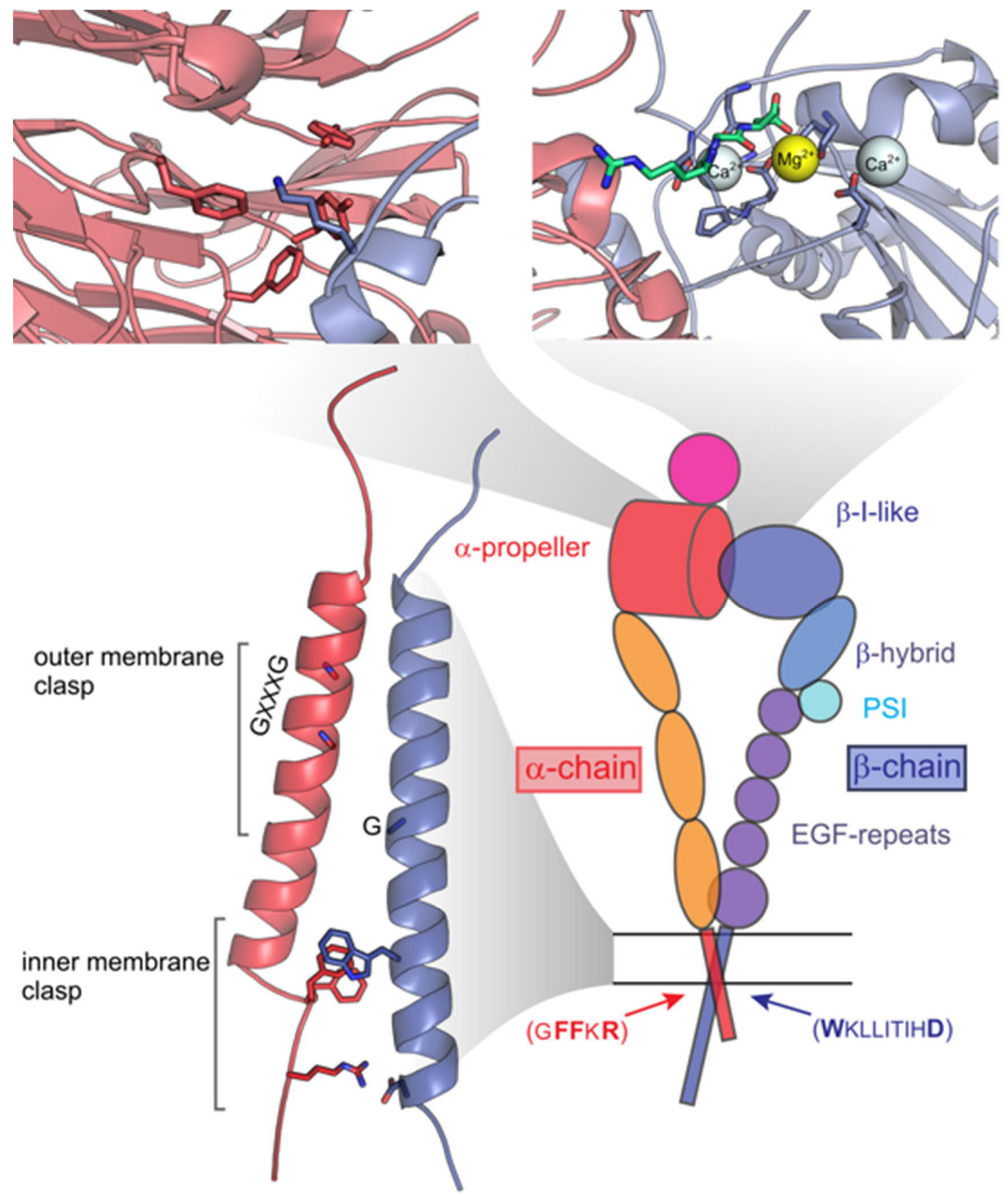

Figure 2 Structural features of the integrin heterodimer.

Integrins consist of $\alpha$ - and $\beta$-subunits, and both of the subunits are membrane anchored. The integrin subunits non-covalently associate in the extracellular domain via the insertion of a conserved lysine residue of the $\beta$-I-like domain (blue) into the aromatic core of the $\alpha$-propeller domain (red) (upper left; respective amino acids are indicated by their structure instead of a cartoon-like representation), locking the subunits tightly together. There are also more dynamic interactions occurring at the transmembrane (outer and inner membrane clasp) and juxtamembrane regions (inner membrane clasp) of the two subunits. Conserved glycine-motifs in both transmembrane domains allow the tight association of the transmembrane domains at the outer membrane clasp, while the inner membrane clasp is stabilized by aromatic interactions. Sequence on the lower right: $\alpha$-subunit: FF; $\beta$-subunit: $\mathbf{W}$ ) and a juxtamembrane salt bridge. Sequence on the lower right: $\alpha$-subunit: $\mathbf{R}$; $\beta$-subunit: $\mathbf{D}$ ); both interactions are highlighted on the lower left by showing the respective structures of the amino acids. 
The cytoplasmic domains contain binding epitopes for a number of proteins, discussed further in Figure 4. The interface between the $\alpha$-propeller in the integrin head piece and metal ions bound to the $\beta$-I-like domain, form a binding site for integrin ligands such as RGD (upper right zoom-in; metal ions from left to right: SyMBS (synergistic metal binding site), MIDAS (metal ion-dependent adhesion site), ADMIDAS (adjacent MIDAS)). In contrast, integrins with an $\alpha-$ I domain in the $\alpha$-subunit bind their ligand only with the $\alpha$-I domain (purple circle in the central cartoon; see also Figure 1). Ligand affinity is modulated by the coordination of the metal ions that includes amino acids of the integrin as well as the ligand (see Fig. 3C for details). Structural information regarding the lysine insertion into the $\alpha$-propeller is from PDB 3VI4 ( $\alpha 5 \beta 1$ integrin (302)), for the MIDAS site from PDB 3ZE2 ( $\alpha \operatorname{IIb} \beta 3$ integrin (501)), and for the transmembrane domains from PDB $2 \mathrm{~K} 9 \mathrm{~J}$ ( $\alpha \operatorname{IIb} \beta 3$ integrin (242)). 
A closed integrin head-piece

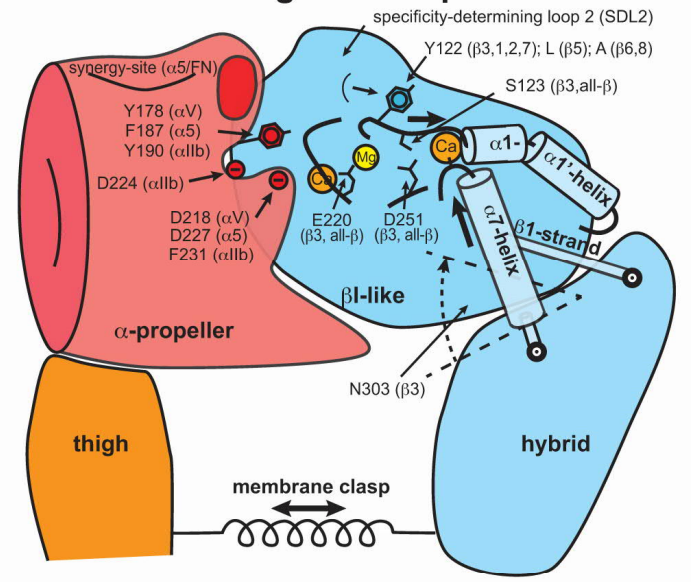

C changes in MIDAS coordination

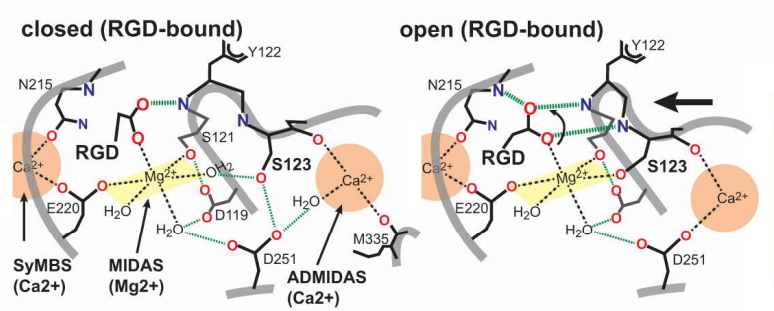

E integrin conformational continuum

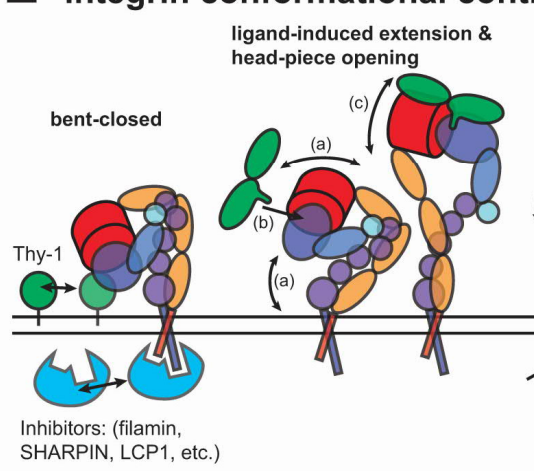

B open integrin head-piece (RGD-bound)

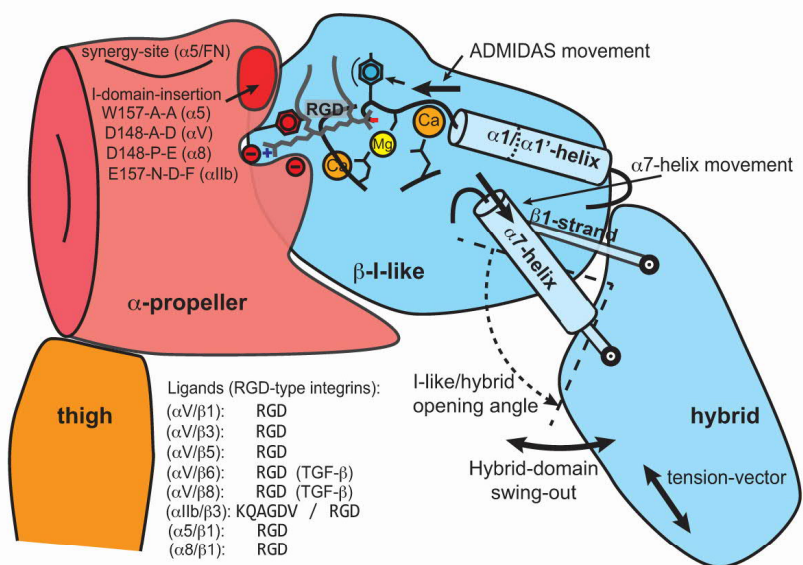

D I-domain containing (closed \& open)

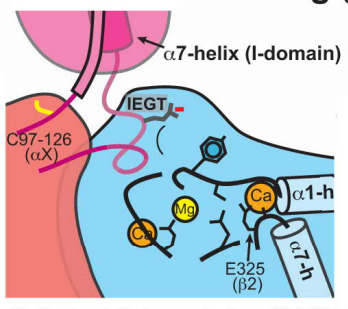

Similar ligand-binding mechanisms: (I/LE/D)-type)
$(\alpha 3 / \beta 1) /(\alpha 6 / \beta 1)$ : LAMC1: CFNTPSIEKP $(\alpha 3 / \beta 1) /(\alpha 6 / \beta 1):$ LAMC1: CFNTPSIEKP
$(\alpha 3 / \beta 1) /(\alpha 6 / \beta 1):$ LAMC2: CYNTQALEQQ

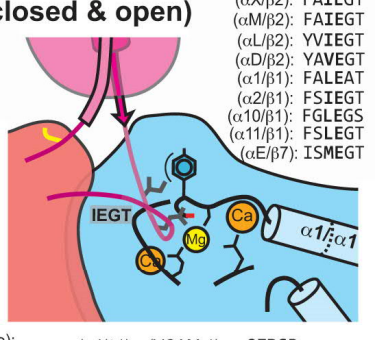

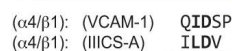

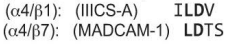

Figure 3 Ligand-induced conformational changes and sequence specific variations in the integrin ectodomain.

Ligne claire drawing of a prototypical integrin headpiece and mechanisms of atomic (C), small (A,B,D) and large (E) scale conformational changes. A) Closed, ligand-free state of the integrin headpiece stabilized by the ADMIDAS (adjacent to MIDAS; see also Figure 2) $\mathrm{Ca}^{2+}$ ion, linking the N-terminal end of the "broken" or "bent" $\alpha 1 / \alpha 1$ '-helix with the $\beta 6$ - $\alpha 7$ helix loop, by coordination (large, bold arrows). In the closed conformation/state, the $\alpha 7$-helix (piston) is tethering the hybrid-domain to form a small $\beta I$-like/hybrid-domain angle. Experimental N-glycosylation at N303 ( $\beta 3$ ) has been used to prevent the formation of the closed integrin head-piece $(90,272)$. The closed head-piece conformation is further maintained by flexible associations of the lower leg domains (see E) by the membrane clasp, as indicated by a spring. Residues relevant for integrin isoform specific RGD-ligand binding of the $\alpha$-propeller domain are shown by arrows (see also Figure 5). Conserved residues involved in MIDAS (metal ion depending adhesion site) coordination are labeled according to human $\beta 3$ (small arrows). Ligand binding specificity in the $\beta$-chain is achieved by the 
specificity-determining loop 2 (SDL2) and residue Y122 (small arrows). For example, SDL2 makes contact with the internal ligand "IEGT" in $\alpha$ I-domain containing integrins (D) $(\alpha X \beta 2$; (391)), with MadCAM in $\alpha 4 \beta 7$ (489), or with the RGDL motif in the TGF- $\beta$ binding integrins $\alpha v \beta 6$ and $\alpha v \beta 8$, while simultaneously avoiding steric clashes with TGF- $\beta$ due to an Ala-residue at the Y122 position $(113,321)$ (see Fig. 5B). Integrin headpiece opening is induced by the Asp-residue binding of RGD at the MIDAS site, which leads to the movement of the ADMIDAS ion and $\alpha 1$-helix straightening towards the MIDAS $\mathrm{Mg}^{2+}$ ion (large arrow), as well as progressively stronger association of the Arg-side chain of the RGD peptide with the $\alpha$-domain. ADMIDAS, S123 side-chain and backbone movement towards the MIDAS ion are linked to a rotation of the carboxyl-group of the RGD, Asp-residue and stabilization by Hbonds (thick dotted green lines in $\mathrm{C}$ ). Due to this movement, the $\beta 6-\alpha 7$ loop detaches from the ADMIDAS $\mathrm{Ca}^{2+}$ ion and induces the piston-like downward shift of the $\alpha 7$-helix, pushing the hybrid domain outwards. The hybrid domain swing-out and opening of the interdomain angle is facilitated by a flexible domain connection at the $\mathrm{N}$-terminus of the $\beta 1$-strand of the $\beta$-I-like domain. RGD-ligand binding and cytoplasmic adapter-mediated link to the actin cytoskeleton result in a tension vector parallel to the hybrid-domain (see also E). C) Close-up view of the SyMBS, MIDAS, and ADMIDAS coordination and their changes during integrin opening. In the closed state, the incoming RGD-ligand coordinates with the MIDAS ion, forming a single H-bond with the backbone amine of Y122. Rotation of the carboxyl sidechain establishes additional $\mathrm{H}$-bonds that progressively induce the movement of S123 and backbone-associated ADMIDAS $\mathrm{Ca}^{2+}$ ion to directly coordinate MIDAS or D251, respectively. These changes in MIDAS and ADMIDAS coordination further stabilize RGDligand coordination as well as the detachment of the $\beta 6$ - $\alpha 7$-loop to induce $\alpha 7$-helix pistoning. D) I-domain-containing integrins show a similar mechanisms of acidic residue binding at the MIDAS (internal ligand peptide: IEGT), but an additional hydrophobic interaction at a preceding Ile-residue ((391), PDB: 4NEN). A similar binding mode, centered around a glutamic acid or aspartic acid residue, has been proposed for laminin-binding integrins (348, 429 ), as well as for $\alpha 4 \beta 1$-binding of the LDV-motif in the IIICS alternatively spliced domain of fibronectin, and the I/L-D-T/S consensus sequence in MAdCAM-1 and VCAM-1 binding to $\alpha 4 \beta 1 / 7$ (489). However, and in contrast to RGD-binding integrins, the binding groove of $\alpha 4$-binding integrins is vertical, binding parallel to the smooth, ligand-facing $\alpha$-propeller surface for the latter integrins (D). E) Ligand-induced conformational changes in the integrin head-piece are proposed to be associated with the head-piece opening of the integrin. The ligand can be external (e.g. fibronectin) or, in the case of the $\alpha$ I-domain containing integrins, be the $\alpha \mathrm{I}$-domain serving as an internal ligand for the integrin headpiece (289). However, physiological ligands might also be recognized in cis, as proposed for Thy-1 (136) or $\beta 2$ integrins and ICAM (131). Alternatively, Adair et al. have demonstrated FN type III9-10 binding to the bent conformation of $\alpha v \beta 3$ (3). For $\alpha 5 \beta 1$, the synergy site on the $\alpha$-propeller surface enhances the FN on-rate (302). A recent survey of integrin ectodomain conformations by electron microscopy demonstrated that the $\beta 1$-class of integrins are more extended in the inactive state compared to $\alpha v \beta 3$ (289). This, however, underlines the importance of the intracellular integrin adapters, as they can either prevent integrin activation, like in the case of sharpin, filamin and LCP1 $(264,359,441)$, or induce leg separation and integrin extension (484), that should subsequently facilitate ligand binding, and full head-piece opening. Note that (a) to (c) indicate a temporal order. An ultimate regulation of the open head-piece conformation could be achieved by mechanical tension, changing the conformational equilibrium of the bound integrins $(31,347,422)$. 


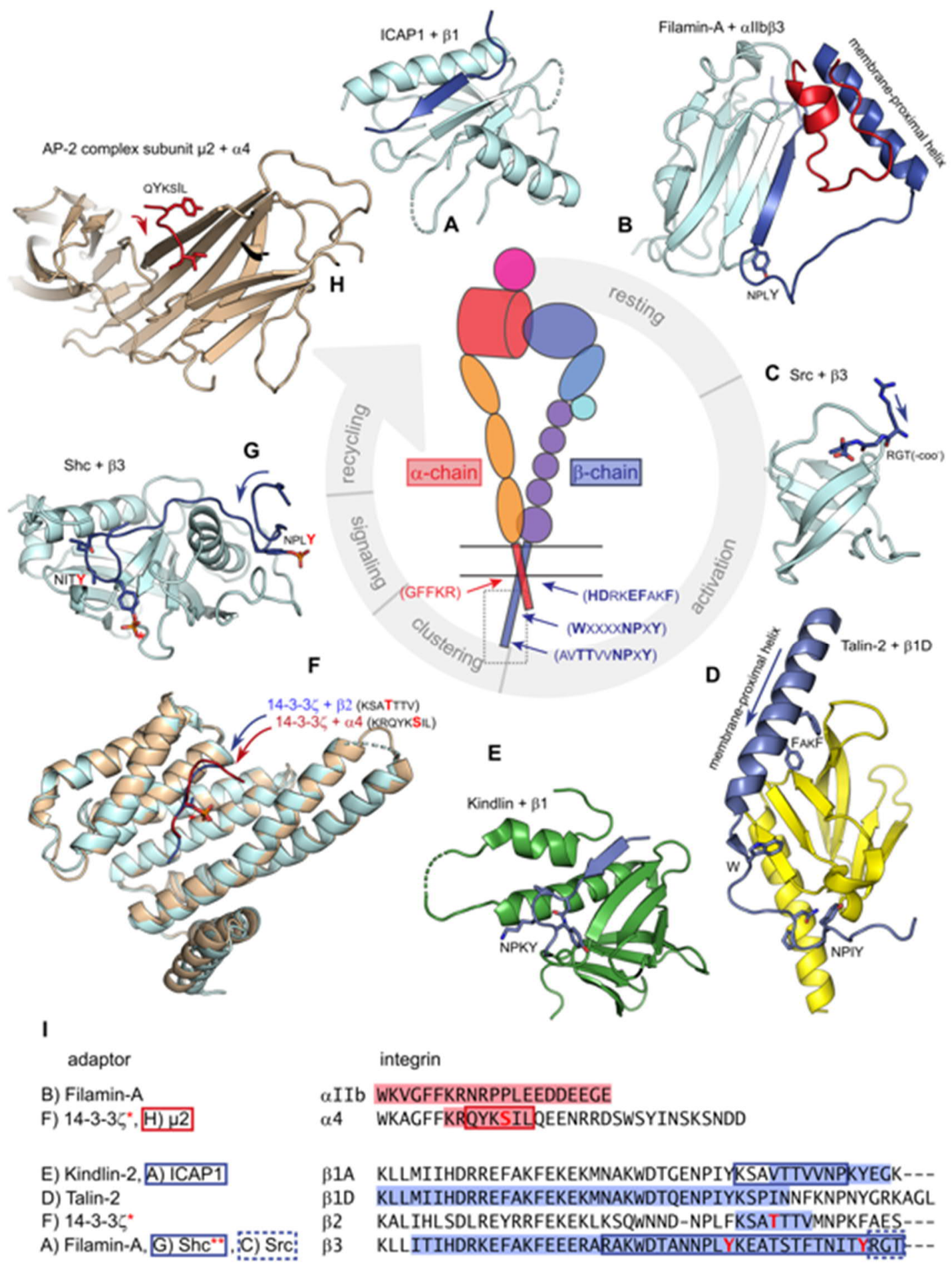

Figure 4 Structural features of integrin ligand binding - intracellular ligands. Cytoplasmic integrin tails comprise only a short part of the complete integrin, but interact with a wide number of adapters (or intracellular ligands; see also Table 1). Here, structures of integrins binding to cytoplasmic adapters are shown together with the amino acid sequence of integrins highlighted in red ( $\alpha$-subunit) or blue ( $\beta$-subunit), indicating the involved amino acids in the binding of the respective adapter. Integrin inhibitors like ICAP or Filamin compete with integrin activators like kindlin or talin for binding to the same domains of the cytoplasmic integrin tail (compare structure A to structure E and structure B to structure D). 
Kindlin binding arranges the membrane-distal part of the $\beta$-integrin tail, potentially priming the integrin for talin binding (30). Talin binding to the membrane-proximal part facilitates the separation of $\alpha$ - and $\beta$-subunit (380) and thereby integrin activation. The respective sequences in the $\beta$-subunit (indicated with blue letters) are highly conserved among integrins. Inactive integrins are stabilized by the interaction of a conserved sequence in the transmembrane part of the $\alpha$-subunit (GFFKR) with the $\beta$-subunit. Integrin inactivation can occur by Tyrphosphorylation of the NPXY-motifs, or alternatively by Ser/Thr-phosphorylation of the inter-NPXY-region, which reduce talin and kindlin association $(14,45)$, while allowing regulatory and recycling adapter recruitment to the integrin receptors. Alternatively, integrins can be internalized bound to their extracellular, but proteolytically fragmented ligands (see Chapter 3; (142, 293, 328)). A) ICAP1 + $\beta 1$ integrin: PDB 4DX9 (267). B) Filamin-A + $\alpha \operatorname{IIb} \beta 3$ integrin: PDB 2MTP (264). C) Src $+\beta 3$ integrin: PDB 4HXJ (469). D) Talin $2+\beta 1 D$ integrin: PDB 3G9W (16). E) Kindlin2 + $\beta 1$ A integrin: PDB 5XQ0 (253). F) $14-3-3 \zeta+\beta 2$

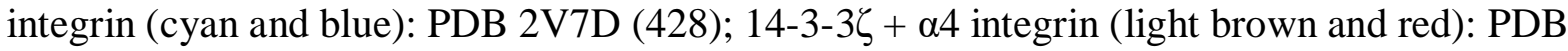
4 HKC (48). G) Shc $+\beta 3$ integrin: PDB 2L1C (108). H) AP-2 complex subunit $\mu 2+\alpha 4$ integrin: PDB 5FPI (141). 

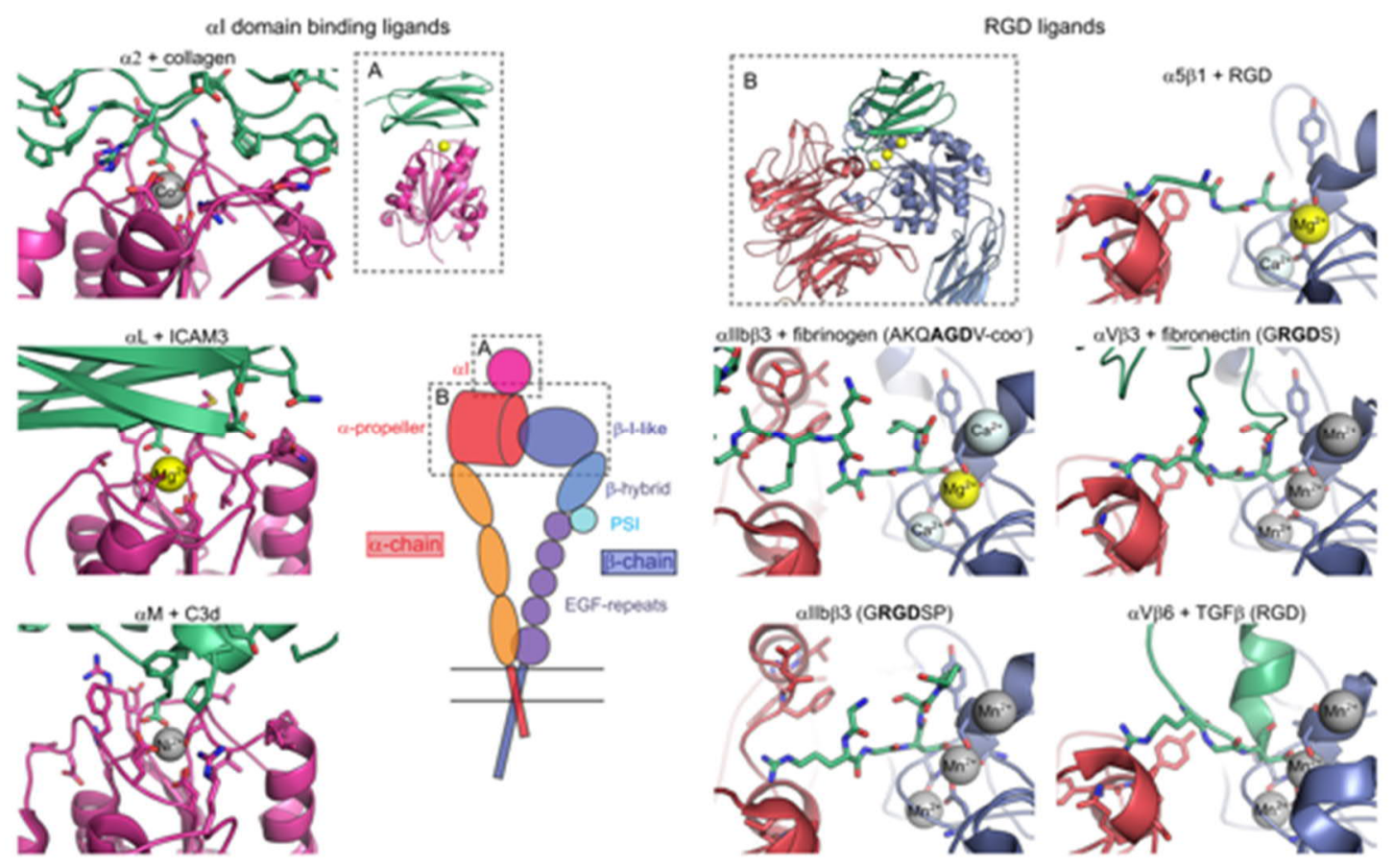

Figure 5 Structural features of integrin ligand binding - extracellular ligands. Integrin-ligand interactions are shown on the left for $\alpha$-I domain containing integrins with the indicated ligands and on the right for integrins without the $\alpha$-I domain. Please note that in the first case only the $\alpha$-I domain (purple) makes contact to the ligand (green), while in the latter the heads of both the $\alpha$ - and the $\beta$-subunit (red and blue, respectively) contact the ligands (green). The overview of different RGD ligands with relevant integrins (right hand side) highlights the structural diversity within this group for both integrins as well as RGD ligands. It is noteworthy that in $\alpha$-I domain integrins only one metal ion site is present (MIDAS), potentially favoring a stricter on-off binding regime due to the missing fine tuning by the ADMIDAS site. The binding pocket for ligands is also more accessible compared to integrins without $\alpha$-I domain (compare e.g. the orientation and peptide presentation of ICAM3 to fibrinogen binding to $\alpha \operatorname{IIb} \beta 3$ ). The accessibility might also have an impact on fibronectin binding, requiring the presentation of the RGD peptide in a short loop to integrins. High-affinity binding to $\alpha 5 \beta 1$ is facilitated by auxiliary binding of fibronectin to the synergy binding site on $\alpha 5$ (302). Lack of this auxiliary binding might contribute to conformation-specific binding of $\alpha \mathrm{V} \beta 3$ integrin to fibronectin. The accessibility is also expected to influence TGF $\beta$ binding; the RGD peptide in LAP/TGF $\beta$ is surrounded by a bulky $\alpha$-helix. Steric hindrance with Tyr122 prevents binding to $\beta 3$ integrins, in contrast to $\beta 6$ and $\beta 8$ (Ala) and $\beta 5$ (Leu) (see also Fig. 2 ). Structures shown: $\alpha 2 \beta 1$ + collagen: PDB 1DZI (124); $\alpha$ L $\beta 2$ + ICAM3: PDB 1T0P (408); $\alpha \mathrm{M} \beta 2$ + C3d: PDB 4M76 (33); $\alpha 5 \beta 1$ + RGD: PDB 3VI4 (302); $\alpha \operatorname{IIb} \beta 3$ + fibrinogen: PDB 2VDO (409); $\alpha$ V $\beta 3$ + fibronectin: PDB 4MMX (5); $\alpha \operatorname{IIb} \beta 3$ + RGD: PDB 3ZE2 (501); $\alpha$ V $\beta 6$ + LAP/TGF $\beta$ : PDB 5FFO (113). 


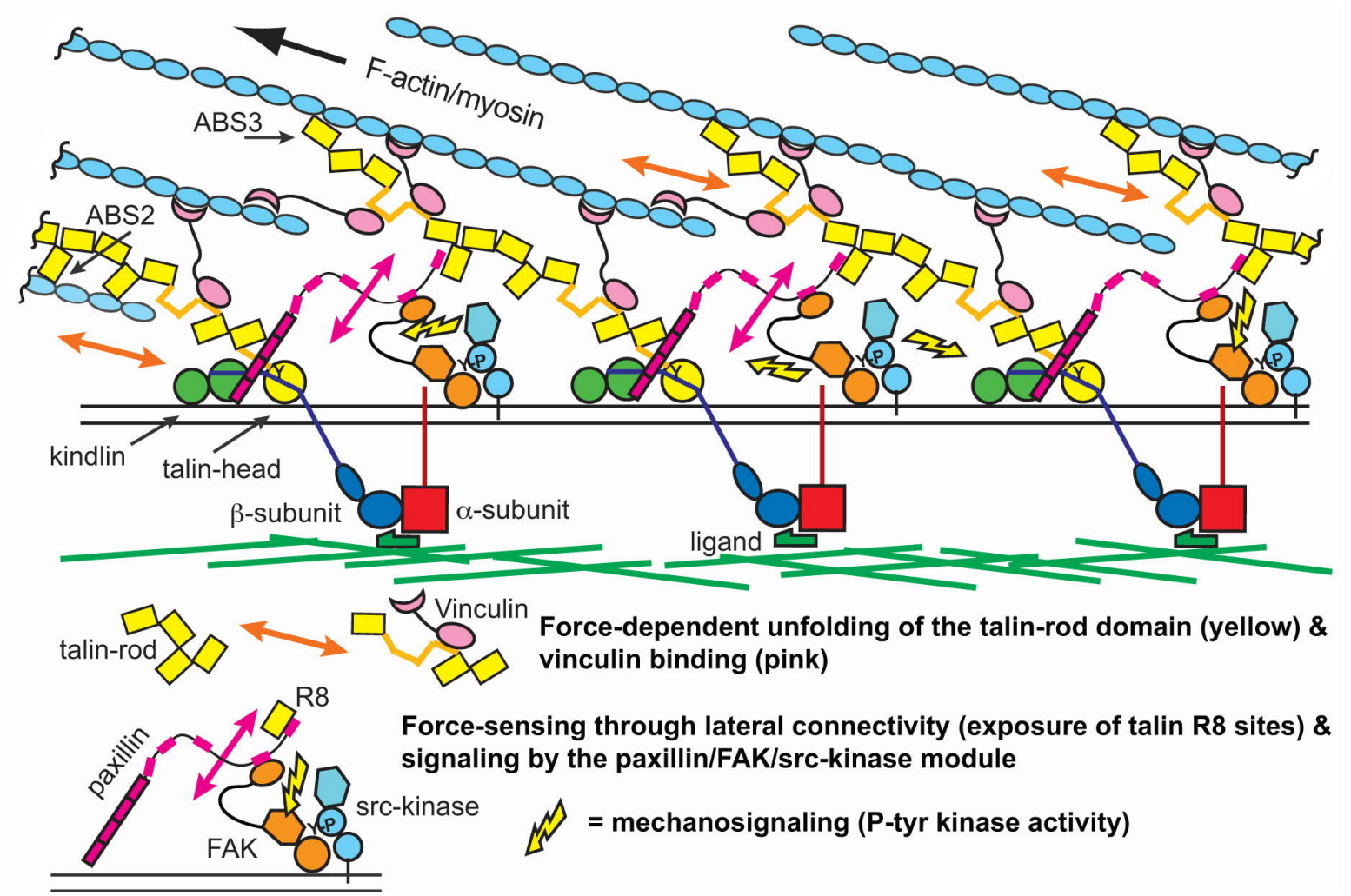

Figure 6 Protein interplay within integrin-mediated adhesion. This schematic figure highlights how protein interactions in cell-matrix adhesions are controlled by conformational regulation of the adhesion proteins. The integrin cytoplasmic domain acts as a binding platform for several proteins. Talin (yellow) binds with its head domain to the cytoplasmic domain of the $\beta$-subunit and with its tail connects it to the actomyosin machinery. The talin tail with its rod domains contains actin binding sites (ABS) for initial integrin-actin linkage (ABS3). Under mechanical load cryptic binding sites of talin open for one or more vinculins (light pink), as well as F-actin (ABS2) reinforcing the mechanical connection between integrin and actin (24). Vinculin (the head domain binds talin, the tail domain binds actin) can also recruit additional adapters and thereby contribute to adhesion signaling (71). Kindlin (green) co-operates with talin in integrin activation and adapter recruitment as for example paxillin (335). Paxillin (purple) is an important scaffolding protein, recruited to focal adhesions via its LIM domains and organizing Rho-GTPase signaling via Paxillin LD domains. This signaling (indicated by a yellow lightning symbol) is modulated by FAK-Src (orange, light blue)-mediated phosphorylation. FAK consists of a focal adhesion targeting domain binding to paxillin, a kinase domain and a FERM domain binding to the lipid membrane. Interactions in this web are very dynamic, with binding and unbinding events in quick succession. Accordingly, a one-to-one pairing is not to be expected. Instead, a mutual connectivity between the tension-defined ECM-integrin-talin-actin axis and perpendicular interactions based on the paxillin/FAK/talin signaling axis will reinforce and stabilize the adhesion-structure (slanted fence model; see Chapter 4). 


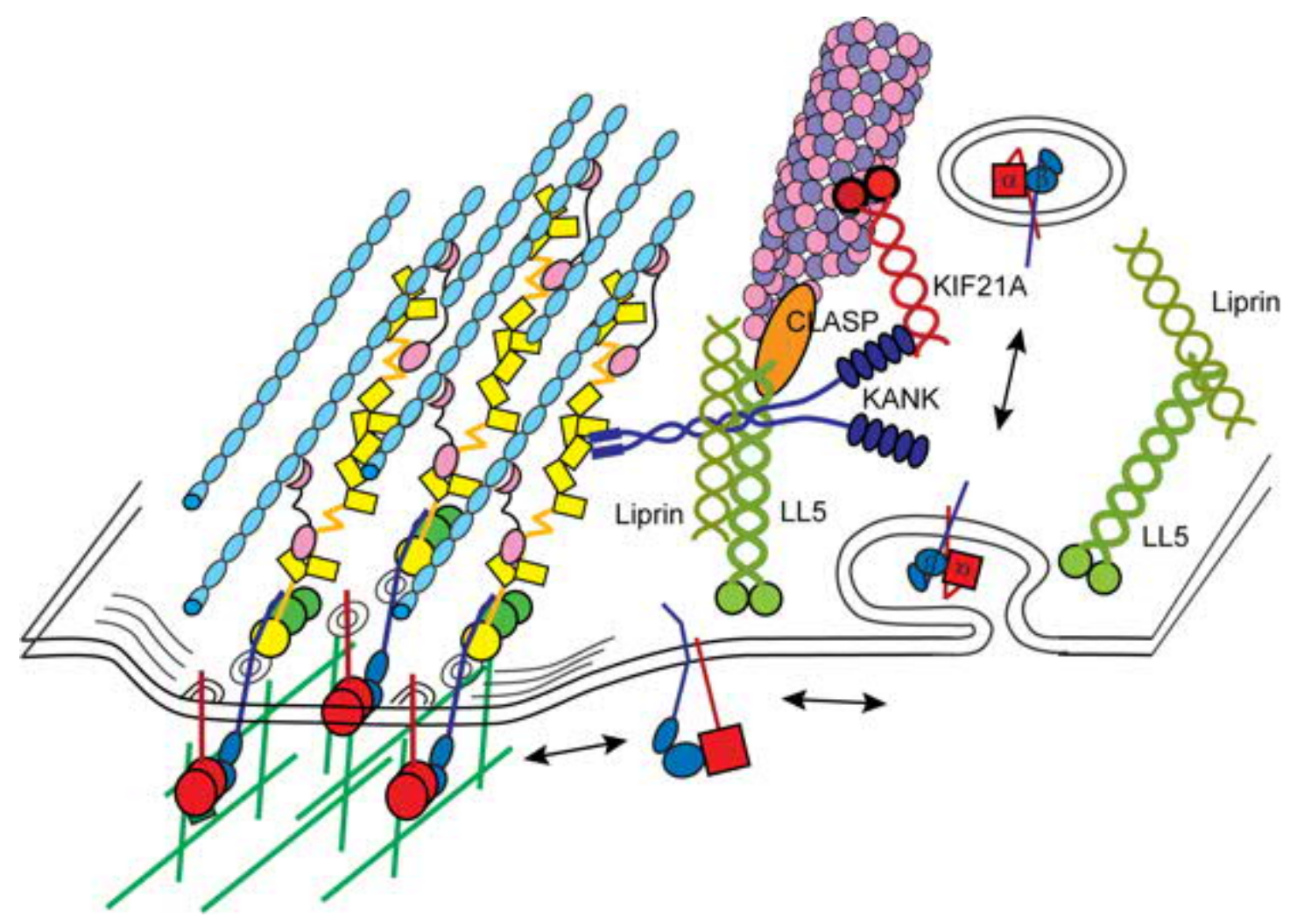

Figure 7 Interplay between focal adhesion and microtubules. Ligand-bound integrins are in closer proximity to the substrate compared to the surrounding membrane. The core of integrin-mediated adhesions is densely populated and might differ from the 'outer shell'. Microtubules and focal adhesions are linked via Kank proteins at this outer shell, which directly interacts with the talin rod domain (52). While kank can destabilize the talin association with F-actin (420), it also links talin to CLASP proteins and the liprin scaffold. CLASP is important for recruitment of microtubules to adhesion sites, which in turn controls the localized release of proteases (410). The link of kank-proteins to the liprin scaffold directs vesicular trafficking to the cell periphery, involved in directed fibronectin release at sites of adhesion to mediate fibrillogenesis (278), as well as to induce cell spreading during cancer cell migration (86). 


\section{Integrin activation cycle}

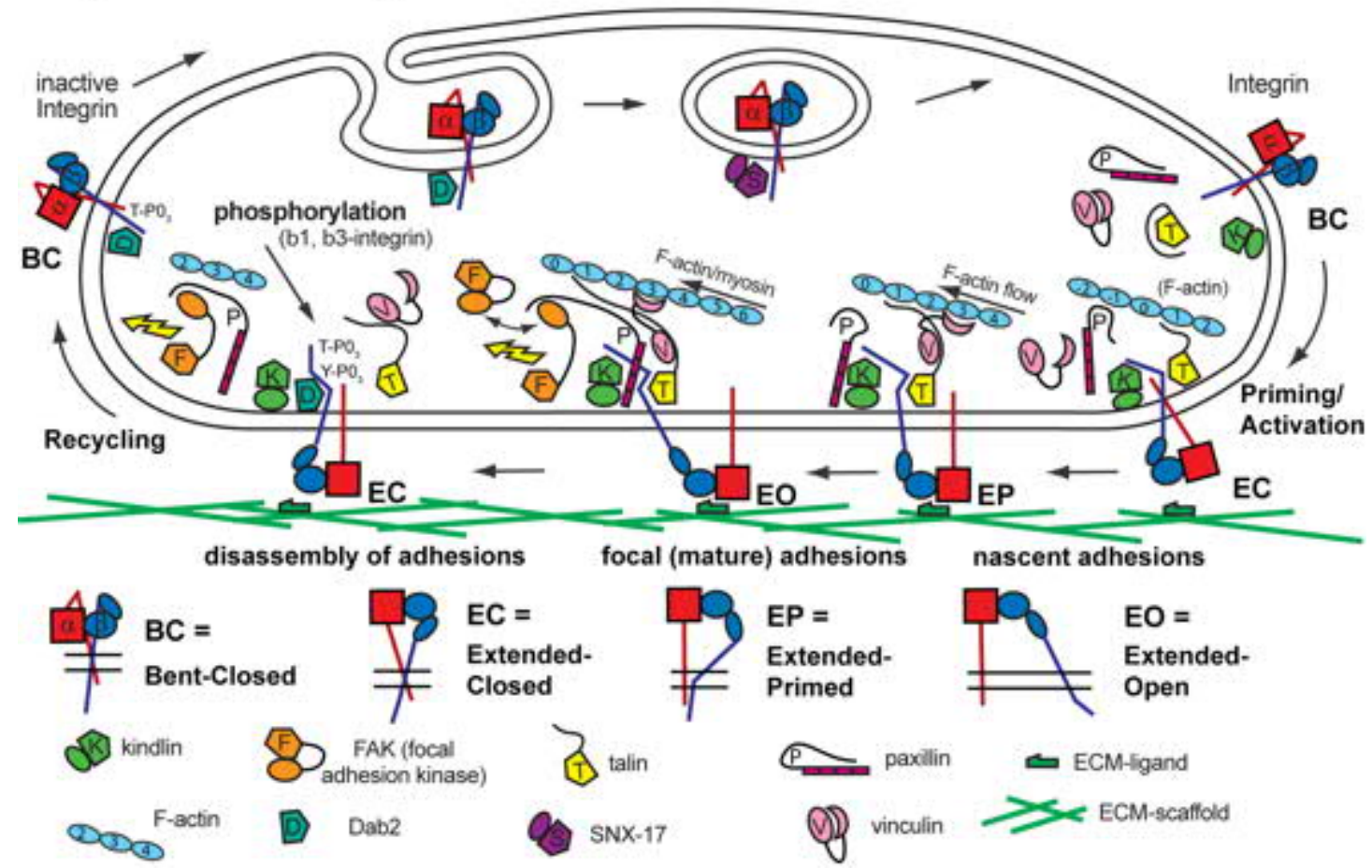

Figure 8 Integrin activation cycle. Integrins show the inactive, bent conformation in absence of intra- or extracellular adapters. The transmembrane domains are closely associated to support this bent structure $(\mathrm{BC}=\mathrm{Bent}-\mathrm{Closed}$ conformation). The activation process may involve both extracellular (ligand) and intracellular (talin, kindlin) triggers, leading to extension $(\mathrm{EC}=$ Extended-Closed $)$ and priming $(\mathrm{EP}=$ Extended-Primed $)$ of the integrin, which facilitates early adhesion events (see also Figure 3). For full activation, integrin legs are separated and arranged in the direction of the actomyosin force-vector $(\mathrm{EO}=$ Extended-Open conformation). The activation of integrins is accompanied by the recruitment of signaling (paxillin, FAK) and structural (talin, vinculin) adapters. Recycling of integrin-mediated adhesions can occur via integrin inactivation (as shown in Figure 4), with integrin phosphorylation being an important step. The release of talin allows binding to endocytosis adapters like Dab2, and subsequent recycling via the sorting nexin 17 (SNX-17) $(35,265)$. Integrins can also be internalized together with their ligands $(397,398)$ potentially influencing their capacity to signal from the endocytotic pathway (11). 


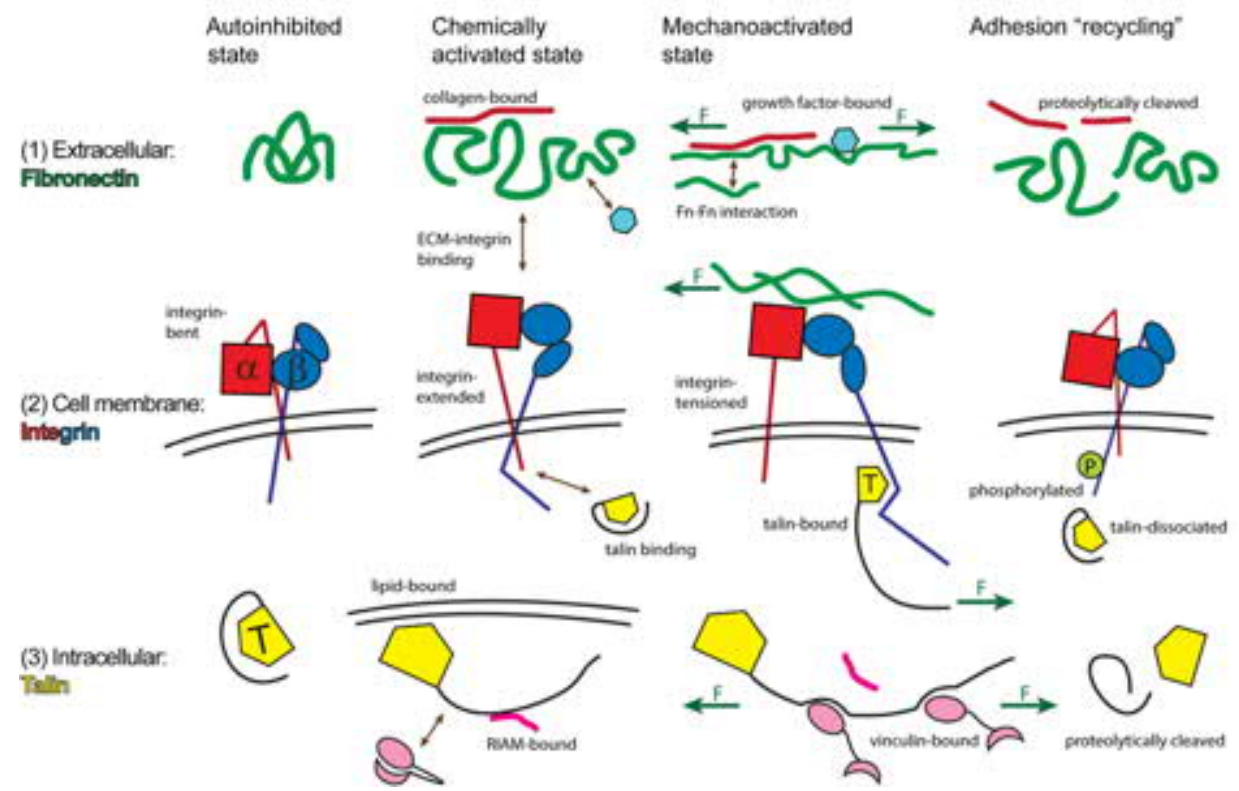

Figure 9 Mechanoregulation of cell adhesion. Many of the proteins participating in cell adhesion have an autoinhibited state. Shown here is fibronectin, which has a globular conformation when the protein is in the soluble state. Inactive integrin shows a closed, bent conformation. Also the intracellular protein talin exists in an autoinhibited state, where the rod domain is in contact with the head domain (163). The first step in the activation process is called "chemical activation", where conformational changes take place, leading to insoluble fiber formation and collagen binding in the case of fibronectin. Integrin activation can be triggered both by extracellular and intracellular signals, such as metal ions (289), RGD-ligand proteins (427) and talin (484). Talin may become activated by lipid-mediated triggers, such as increased PIP2 concentration $(262,380)$. Some of the ligands such as RIAM $(170,244)$ and DLC1 (491) bind only to relaxed talin. For full maturation, the adhesion complex requires mechanical signals. This leads to changes in the properties of ECM, controlling e.g. the binding of growth factors (317). In the case of integrin, mechanical load is needed for the complete activation, where leg separation takes place (500). The mechanosensory role of talin is studied widely and it involves the exposure of binding sites for other adapter proteins, including vinculin (206, 365). Additionally, mechanical tension also leads to dissociation of certain binding partners such as RIAM and DLC1 (170, 178). Finally, to recycle the adhesion, cells may utilize several molecular mechanisms. Proteolytical cleavage of ECM and intracellular proteins may be involved in the adhesion disassembly $(4,144,221)$. Phosphorylation of integrin can be used to tune the binding affinity towards various intracellular partners (14). 